\title{
A tale of two hashtags: More than absolute opposition in an affinity space formed by sexual harassment
}

\author{
by
}

Ashleigh Feltmate

A thesis submitted to the Faculty of Graduate and Postdoctoral Affairs in partial fulfillment of the requirements for the degree of

Master of Arts

in

Applied Linguistics and Discourse Studies

Carleton University

Ottawa, Ontario

(C) 2021

Ashleigh Feltmate 


\begin{abstract}
Digital activism on social media has a strong relationship with hashtags and this activism on social media tends to produce multiple protest and counter-protest hashtags. This case study explores the discursive constructions across competing hashtags (\#KickVic and \#IStandWithVic) that represent the protest and counter-protest surrounding the sexual harassment allegations of Vic Mignogna. Through the synergized approaches of corpus linguistics and critical discourse studies, I investigate the perpetuation of patriarchal ideologies within this affinity space. I constructed three tweet-based corpora for analysis: \#KickVic, \#IStandWithVic, and mixed. Keywords, semantic themes, and discursive constructions of women demonstrate that these hashtags subvert and perpetuate a patriarchal social order. While the perpetuation of these ideologies is more present within the \#IStandWithVic corpus and the mixed corpus, tweeters also use \#KickVic to perpetuate them. Discussion and contributions to affinity spaces and discursive constructions of these digital activism hashtags are included in this investigation. Keywords: hashtags, digital activism, sexual harassment, feminist critical discourse analysis, corpus linguistics
\end{abstract}




\section{Acknowledgements}

In the thirty months since this project's conception, I have received bounties of help and advice from various individuals that I owe all my thanks. To everyone whom I have talked to this project about: thank you. I would not be here without all your unwavering support.

First and foremost, thank you to my fiancé Nicholas for helping me in so many ways on this project. From providing continuous emotional support when finishing this thesis felt impossible, fixing my laptop when the hard drive crashed and suggesting Microsoft Excel functions that made the data sorting process much shorter than it would have taken me to figure out on my own. Had you not been here with me on this journey, I am positive that I would have thrown my laptop out the window from frustration.

This project also owes a lot to my amazing supervisors, Dr. Jaffer Sheyholislami and Dr. Rachelle Vessey, for their incredible insights that helped guide this project into what it is today. Throughout the duration of this project, I truly felt blessed to have such a wonderful team offering advice every step of the way, especially when it came to the theoretical and methodological portions of this thesis. I am thankful for your motivation as well during this turbulent year and a half and for always taking the time to remind me in our meetings to remember what made me passionate about this project in the first place.

Lastly, I give an extended thank you to my MA ALDS peers Alicia and Tarah. Even with 1,400 kilometres between us, we continued to support each other during this challenging time in ways that I will always cherish. I owe you both the biggest hug when we can meet up again! 


\section{Table of Contents}

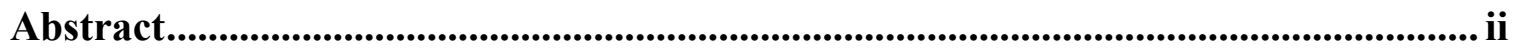

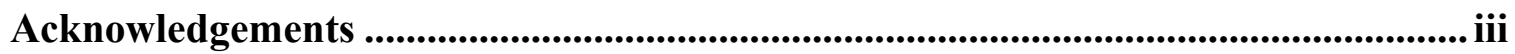

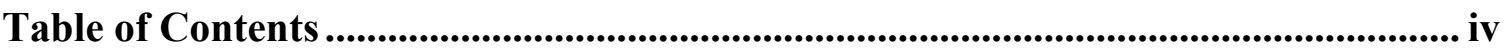

List of Tables ................................................................................................................................ vii

List of Appendices................................................................................................................. viii

Chapter 1: Introduction ................................................................................................................ 1

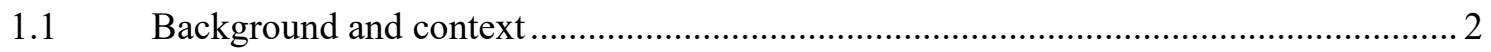

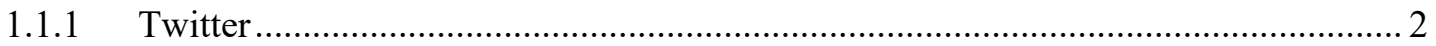

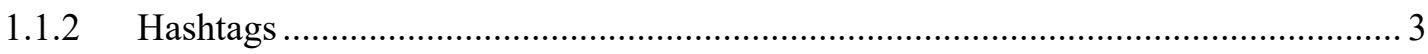

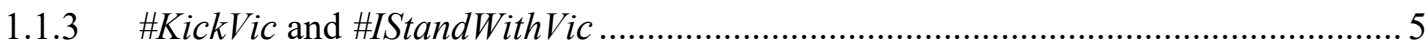

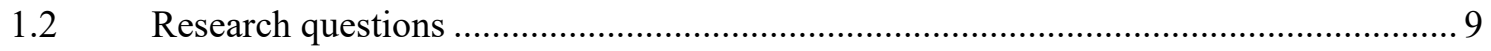

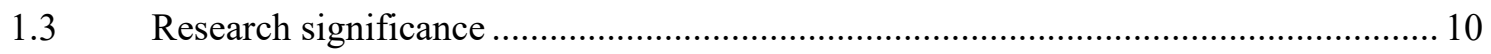

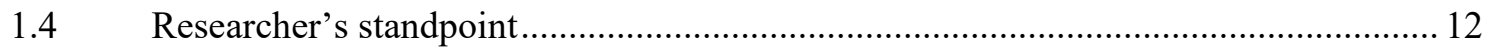

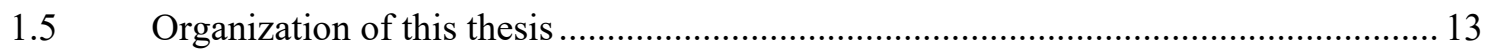

Chapter 2: Literature Review............................................................................................... 14

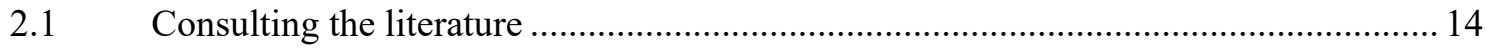

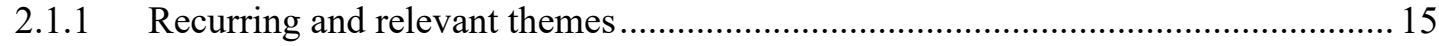

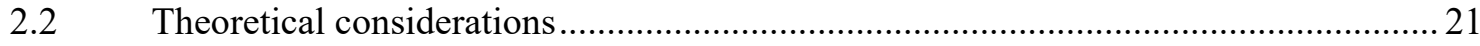

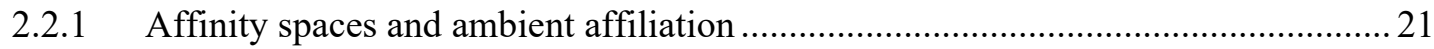

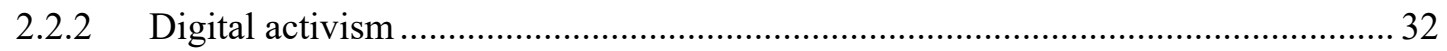

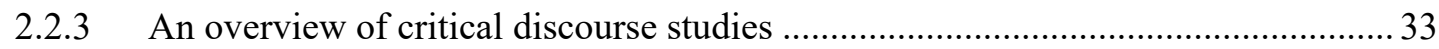

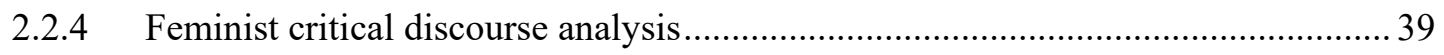




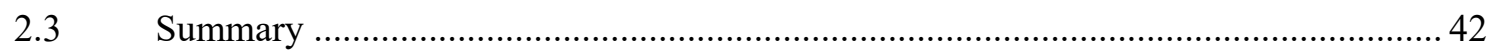

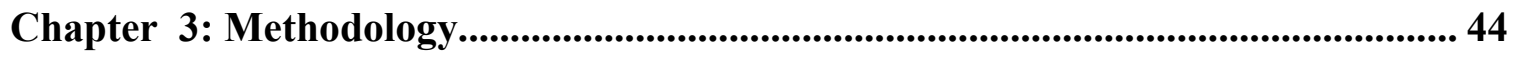

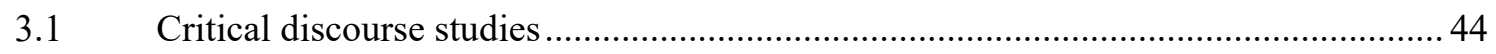

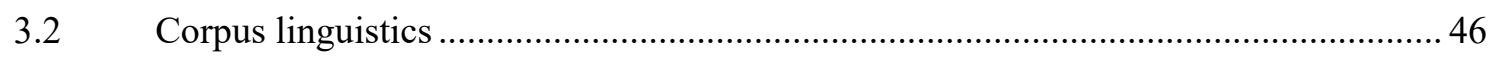

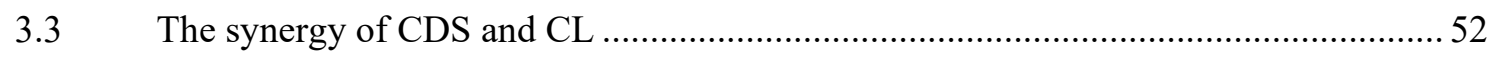

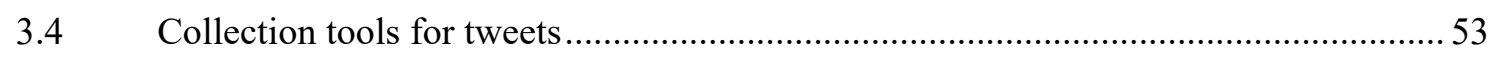

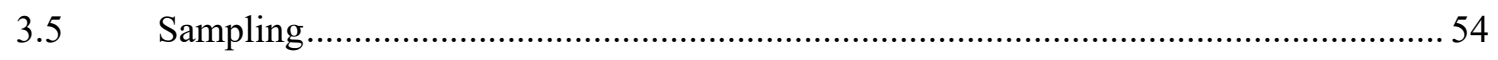

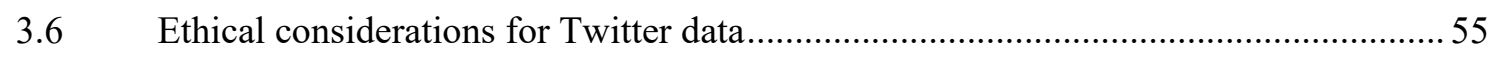

3.7 Cleaning and sorting tweets: A necessary struggle …..........................................5 59

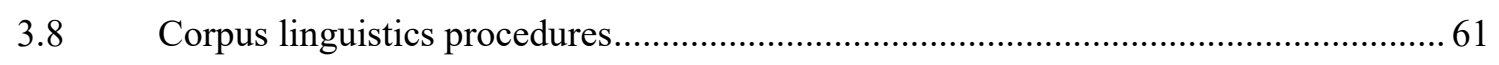

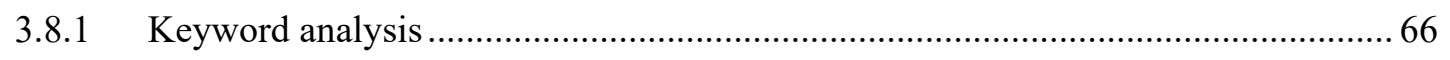

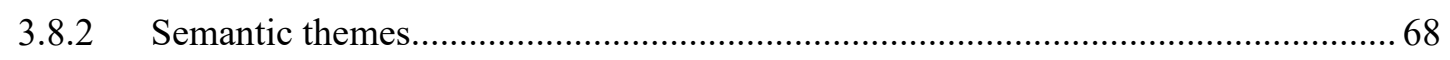

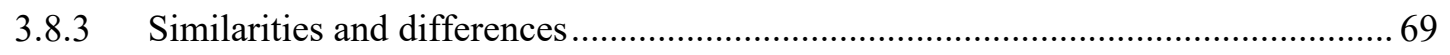

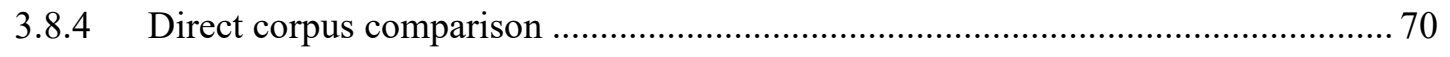

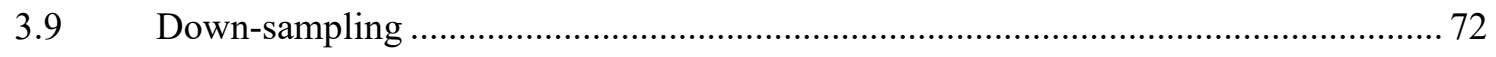

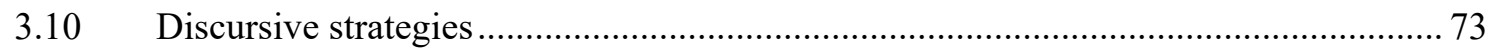

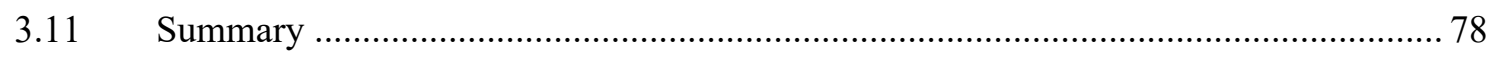

Chapter 4: Findings ......................................................................................................... 79

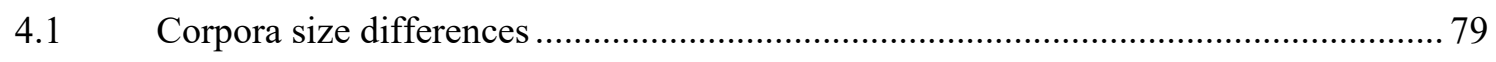

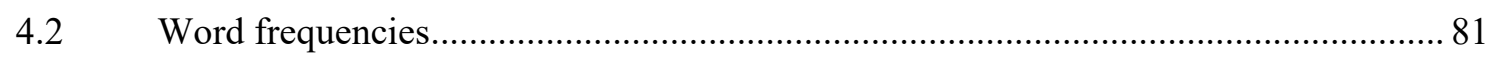

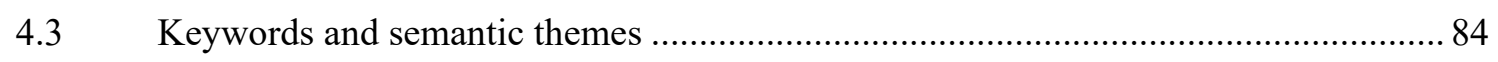

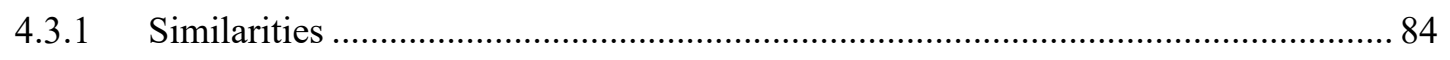

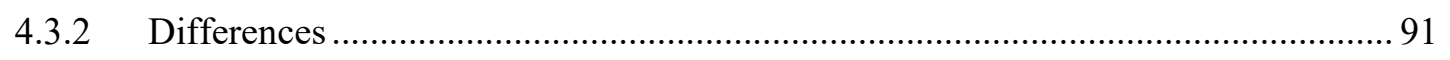

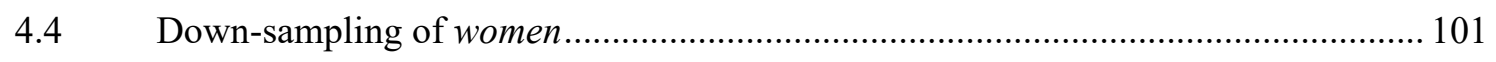

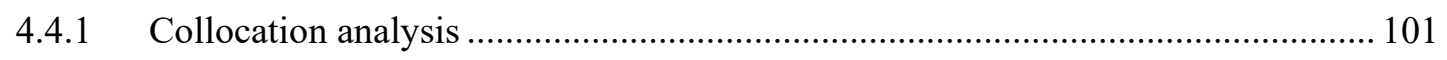




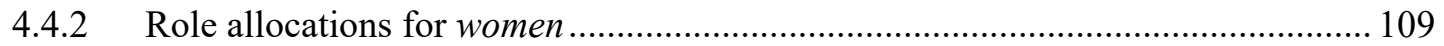

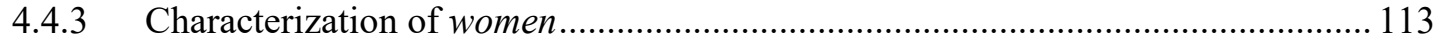

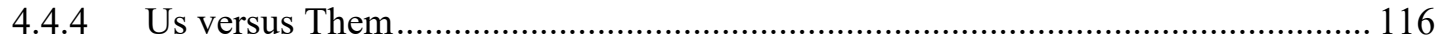

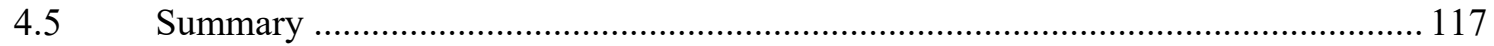

Chapter 5: Discussion ................................................................................................................ 119

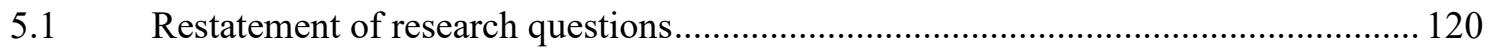

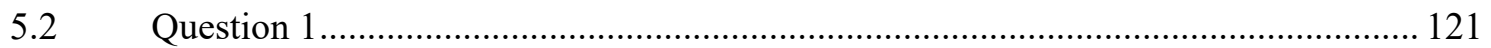

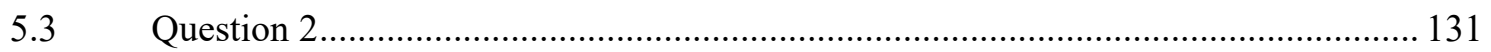

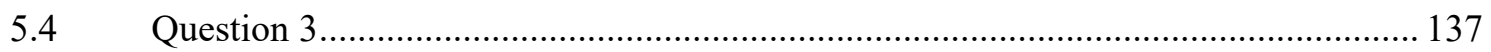

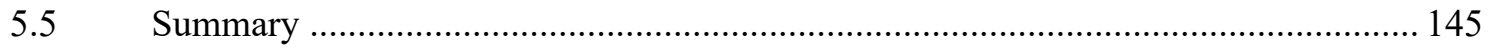

Chapter 6: Conclusion .......................................................................................................... 147

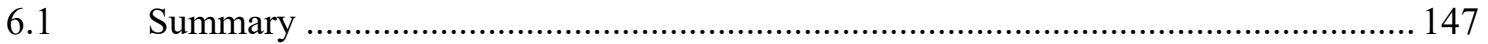

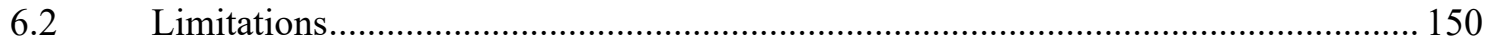

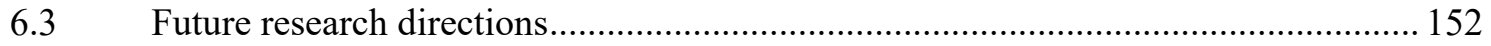

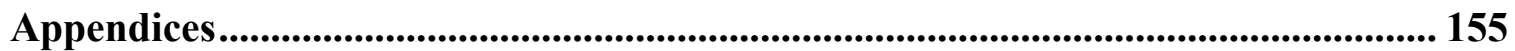

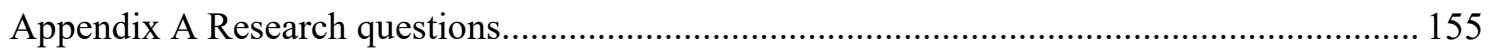

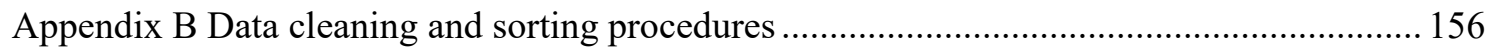

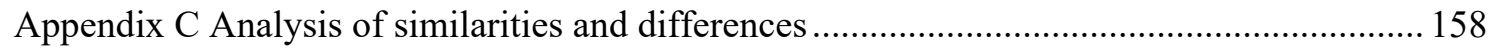

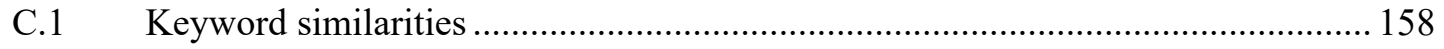

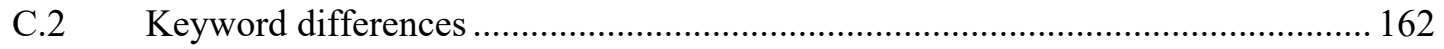

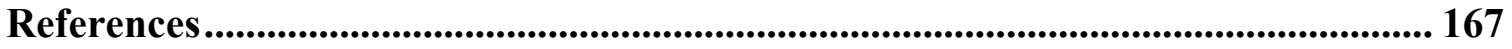




\section{List of Tables}

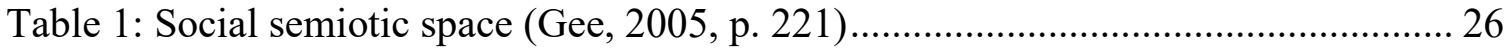

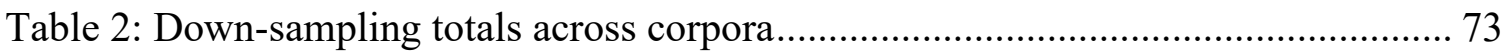

Table 3: Discursive strategies in the DHA (Reisigl, 2017, p. 52) ..................................... 75

Table 4: Raw frequencies of gendered word tokens across corpora................................. 82

Table 5: Normalized frequencies of gendered word tokens across corpora ….................. 82

Table 6: Rankings of gendered word tokens by corpus according to raw frequencies..... 84

Table 7: AmE06/BE06 comparison: Shared across three corpora.................................... 85

Table 8: Totals of unique keywords for each corpus..................................................... 98

Table 9: Totals of similar keywords for each corpus comparison .................................... 99

Table 10: Collocates for women across corpora ………................................................ 102

Table 11: Collocates for woman across corpora .......................................................... 102

Table 12: Totals of women as actors and objects in the \#KickVic corpus ...................... 108

Table 13: Totals of women as actors and objects in the \#IStandWithVic corpus............ 109

Table 14: Totals of women as actors and objects in the mixed corpus ........................... 109

Table 15: Totals of activated/passivated social actors who are women .......................... 110

Table 16: Active forces of women across corpora ...................................................... 112

Table 17: Active forces women undergo across corpora ............................................... 113

Table 18: Totals of individualized/assimilated social actors who are women ................ 114

Table 19: Totals of categorized/nominated social actors who are women ...................... 116

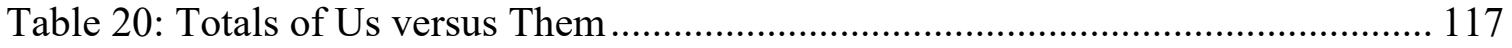




\section{List of Appendices}

Appendix A Research Questions............................................... 155

Appendix B Data cleaning and sorting procedures.............................. 156

Appendix C Analysis of similarities and differences........................... 158

C.1 Keyword similarities.......................................... 158

Table C.1.1 AmE06/BE06 comparison: Shared between \#KickVic and \#IStandWithVic............................................ 158

Table C.1.2 AmE06/BE06 comparison: Shared between \#KickVic and mixed................................................. 159

Table C.1.3 AmE06/BE06 comparison: Shared between \#IStandWithVic

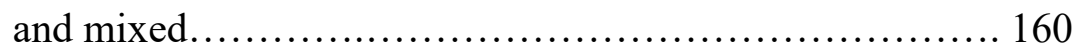

Table C.1.4 Mixed corpus as reference: Keywords shared between \#KickVic and \#IStandWithVic............................. 160

Table C.1.5 \#IStandWithVic corpus as reference: Keywords shared between \#KickVic and mixed.............................. 161

Table C.1.6 \#KickVic corpus as reference: Keywords shared between \#IStandWithVic and mixed................................. 161

C.2 Keyword differences........................................... 162

Table C.2.1 AmE06/BE06 comparison: Distinct to \#KickVic............. 162

Table C.2.2 AmE06/BE06 comparison: Distinct to \#IStandWithVic....... 162

Table C.2.3 AmE06/BE06 comparison: Distinct to mixed................ 163 
Table C.2.4 Corpora comparison: Distinct to \#KickVic................... 164

Table C.2.5 Corpora comparison: Distinct to \#IStandWithVic..............165

Table C.2.6 Corpora comparison: Distinct to mixed corpus............... 166 


\section{Chapter 1: Introduction}

Founded and launched in 2006, the microblogging and social networking service Twitter has become a public platform for more than tweets, likes, and hashtags. Arguably one of the most prevalent and recognizable hashtags to date, \#BlackLivesMatter is one example of an arranged spontaneous protest with online origins that aims to critique the power imbalances embedded into our societies to enact social change. This thesis investigates the discursive constructions of one such instance of protest in conjunction with its counter-protest through the synergy of corpus linguistics (CL), critical discourse studies (CDS), and feminist critical discourse analysis (FCDA). More specifically, the present study examines the similar and unique discursive constructions of three corpora built around the hashtags \#KickVic and \#IStandWithVic to understand if, and how, the discursive constructions of these hashtags subvert or perpetuate dominant ideologies related to women and sexual harassment. This research represents a case study for hashtags used in the service of digital activism, and the data collected constitutes a selection of tweets concerning the sexual harassment claims attributed to the North American (NA) voice actor Vic Mignogna (henceforth Mignogna). Also, my study aims to uncover more information concerning what the hashtag as a tool means for instances of digital activism, specifically for a protest and counter-protest. In this chapter, I provide the relevant information needed to understand the background and trajectory of this thesis. Firstly, I foreground the importance of this research by detailing the contextual information required to interpret the hashtags under investigation. In Section 1.2, I list the research questions that guide this study. Thirdly, I discuss the significance of this project and its research questions as these aspects relate to the pre-existing literature and 
theoretical concepts that inform my thesis. In Section 1.4, I provide a standpoint that explains my stance towards the context of this project, given that CDS and FCDA inform this research and every practitioner will have a bias to what they investigate. Lastly, I outline in section 1.5 the organization of this thesis and the principal elements of all the following chapters.

\subsection{Background and context}

Given the contextual complexity of the discursive constructions that this project investigates I explain the background to the protest and counter-protest in this section, starting at the general information and working towards that which is more specific. This explanation includes how scholars have conceptualized Twitter (Section 1.1.1) and hashtags (Section 1.1.2) to acquaint the reader with this means of online interaction. In Section 1.1.3, I explain what voice acting is and how the industry relates to anime conventions. Within this section, I also summarize the protest and counter-protest hashtags, including how and why they began as well as the efforts of this digital activism.

\subsubsection{Twitter}

Often associated with the larger category of social media, Twitter-a microblogging and social networking service-is the origin of what we know today as the hashtag. With 330 million users in quarter one (Q1) of 2019, people recognize Twitter for its logo (a white bird on a light blue background that appears to be tweeting) and its strict character constraints (Iqbal, 2021). After all, it is known as a microblogging service; since its launch in July 2006, Twitter allowed users to post updates of 140 characters or less, which included everything from spaces to usernames. Over time, a lot has changed about the character limit of posts, with the biggest being that in 2017, the 
character limit was doubled to 280 for all users, meaning that while messages were still short, they could contain more content now.

The posting of a message on Twitter is referred to as tweeting; the message is also called a tweet (tweets is the plural form). Concerning Twitter's layout, User A can follow Users B, C, and D because User A wants to see what Users B, C, and D tweet. However, Users B, C, and D do not necessarily have to follow User A back. Twitter users cultivate a customized stream of content on the microblogging service which takes the form of a timeline. The timeline contains tweets from accounts that User A follows and tweets User A has made themself or retweets: a message that User A chose to reshare from another account.

For Twitter's interaction system, users can engage with the message of a tweet with a like, can respond to a tweet with a reply, and can share tweets to their followers with a retweet. These three forms of interaction are available to most tweets on the platform; this is not possible for tweets from a private account or if the user has specified who can reply to their tweets (everyone, people they follow, or only people who are tagged in the tweet). With this system, users can interact with the accounts they follow, the tweets these accounts make and tweets from accounts they never knew existed until that point. This design makes Twitter a very dialogic background that allows messages of various content to be shared widely amongst users who may not know one another.

\subsubsection{Hashtags}

Considering Twitter as a fluid space where users can freely interact with most of the content that exists on the platform, so too do we find strategies that users have implemented to make their online interaction faster, easier, and more accessible. The 
hashtag - which started on Twitter but is now also a part of other social media platforms like Facebook and Instagram - is one such convention. Although not a part of the original design plan of Twitter, hashtags "grew out of a proposal by Messina (2007) to introduce a system to tag and track content on the site" (Scott, 2015, p. 12). The first hashtags were called channel tags and allowed users to eavesdrop on other conversations about a specific topic; it was not until 2009 that hashtags became an official feature of Twitter, three years after the platform was founded. This convention that links posts (tweets) together have been theorized widely, including a typographic convention (Zappavigna, 2011), searchable talk (Zappavigna 2015, 2018; Zhu, 2016), networks (Clark, 2016), an indexing system (Bonilla \& Rosa, 2015), a digital linguistic practice (Heyd \& Puschmann, 2017), a discourse marker (D’Ambrosio, 2019), and metadata tags (Scott, 2015). The word hashtag is a compound word composed of the word hash, one way to refer to the number sign (\#), and tag because it functions as a tag or index marker. From Twitter's Help Center (n.d.), the only rule for hashtags is that they cannot contain spaces or punctuation; including these will result in the hashtag not hyperlinking properly. Apart from this, anything can be a hashtag, and a tweeter can include it anywhere in a tweet.

Besides how scholars have conceptualized hashtags, they have also identified several different functions that hashtags can have, depending on the context people use them in. These functions include, but are not limited to, that hashtags serve as metadata, or data about data (Zappavigna 2011, 2015), they become discursive spaces for sharing and interaction (Zappavigna, 2011; Bonilla \& Rosa, 2015; Scott, 2015), and they allow individuals to evaluate their morals and stances (Evans, 2016; Giaxoglou, 2018; RightlerMcDaniels \& Hendrickson, 2014). This list is not exhaustive, and tweeters do not use all 
hashtags for the same functions. Keeping the above in mind, I provide an in-depth discussion of hashtag functions as they are relevant to this project in the first part of chapter 2 (Section 2.1), where I synthesize previous studies that inform the present one.

\subsection{3 \#KickVic and \#IStandWithVic}

Before explaining how this hashtag started, I must explain a few contextual elements related to the event so that it is possible to interpret the full depth of the story. Because I belong to this space - which I consider a fan of western anime voice acting and conventions - most of these explanations come from my understanding of these elements from over ten years of exposure and experience to them. For those unaware, anime to the Western world refers to hand-drawn or computer animation originating from Japan. This definition differs from the origins of the word anime, as in Japanese it is a shortened form of the word animation that refers to all animated works and not only that made in Japan. For the remainder of this project, when I use the word anime, I mean the Western concept of it, i.e., animated works that are made in Japan. Since anime is produced in Japan, the audio, or voice-over, is in Japanese, and therefore a watcher who does not understand the language would require subtitles to obtain a more complete understanding of what is happening. Other words for voice-over include voice acting and dubbing. Voice-over is everywhere — e.g., commercials, cartoons, audiobooks — and is a recognized profession for many individuals whom I refer to as voice actors from this point forward. According to Zuleika (2019), anime voice-over "[goes] back to the early '60s when anime made its way to the [United States]." For myself, English dubs of shows like Pokémon and Cardcaptor Sakura were my first exposure to anime as they seemed no different than the American cartoons that also played on television. The list of anime that receive English 
dubs grows every year, and now "distributors [see] the value of hiring experienced voice actors and scriptwriters" to adapt these works (Zuleika, 2019). One such distributor is Funimation, an entertainment company that is "a wholly owned division of Sony Pictures Television" and has been dubbing and distributing anime since 1994 (Rodgers, 2019). As I explain below, the reason I mention this company is because of their involvement in this protest and counter-protest.

Related to English dubs of anime and the context of this protest and counterprotest are anime conventions. An anime convention is an event, sometimes taking place over multiple days, where fans of anime, manga, gaming, and Japanese culture gather at venues (e.g., convention centres, hotels, and college campuses) to enjoy activities and panels related to the above interests. A common aspect of many anime conventions in North America (NA) is to have voice actors from the dubbing industry attend as guests for the event. As guests, voice actors can hold autograph sessions and photograph events, host question and answer (Q\&A) panels and interact with their fans. Importantly, Mignogna is a voice actor, known for roles as Edward Elric from the Fullmetal Alchemist series, Broly from the Dragon Ball films, and Tamaki Suoh in Ouran High School Host Club. According to websites FanCons.com (2019), Mignogna has 447 confirmed guest appearances that date back to 2003 and include conventions in Australia, Canada, Denmark, Ireland, New Zealand, the United Arab Emirates, the United Kingdom, and the United States of America.

The reason behind the explanation of these elements is because the NA voice acting industry and anime conventions are part of this protest and counter-protest which were formed by sexual harassment allegations gaining awareness through digital activism 
hashtags. Existing years before the \#KickVic hashtag began, people have talked about voice actor Vic Mignogna's inappropriate behaviour with underaged individuals at conventions ( Sports Lesbian 0 , 2019). The oldest of these allegations predates Mignogna's career in the English voice acting industry, where "a former [theatre] student and fellow parishioner at Mignogna's church in Virginia alleged" that at the age of sixteen Mignogna behaved inappropriately and touched her non-consensually in his home (Loveridge, 2019b). It took until 2019 for these allegations to come together on Twitter following the premiere of the English dubbed movie Dragon Ball Super: Broly on January $16^{\text {th }}, 2019$, in which Mignogna voices the title character. Following the development of the \#MeToo hashtag, the \#KickVic hashtag started because alleged victims of Mignogna's sexual harassment and the supporters of those victims decided to not remain silent for any longer and instead rally together with the hashtag to hold Mignogna "accountable for his actions" (Wade, 2019). As convention-goers detailed "times when [Mignogna] acted flirtatiously towards them (fondling, kissing, groping, etc.) without their consent," organizers of conventions "shared stories of [his] unprofessional [behaviour], such as overstaying his panel time and yelling at staffers" (Radulovic, 2019). During this time, voice actresses who were colleagues of Mignogna also posted about their past encounters with him on social media, describing that not only had he forcibly touched them non-consensually through hair pulling and kissing but also that they had witnessed him "[perform] the same actions to both colleagues and fans" (Pineda, 2019). This outcry for accountability from victims and their supporters was met with success in the form of change a little over two weeks after the \#KickVic digital protest began. On February $2^{\text {nd }}, 2019$, the production company Rooster Teeth issued a 
statement indicating that Mignogna would "no longer [be] a part of the cast of [the] RWBY [web series] and Rooster Teeth is ending all associations with [him]" (Loveridge, 2019a). As well, the dubbing and distribution company Funimation publicly tweeted on February $11^{\text {th }}, 2019$, that they would "not be engaging Mignogna in future productions" and that they would recast his current role in an anime (Funimation, 2019). At the beginning of 2019, Mignogna was scheduled for "18 convention appearances [,]" however, since the increase in allegations started, "all but five of his appearances [were] cancelled" (Loveridge, 2019a; Martinez, 2019). As alleged victims continued to come forward with their experiences, other voice actors in the industry shared their support for them; the digital protest was fulfilling its ostensible goal of raising awareness and demanding accountability.

However, this digital protest was met with backlash. As victims raised their voices with a hashtag, so too did Mignogna's supporters with one of their own: \#IStandWithVic. This hashtag was created on January $18^{\text {th }}, 2019$, the day after \#KickVic began (January $\left.17^{\text {th }}, 2019\right)$ and became a space to support Mignogna and agree that any allegations made towards him are false. On top of this, Mignogna's supporters donated “more than $\$ 236,000 "$ to an account on the crowdfunding platform GoFundMe that allowed him to sue Funimation, "two female voice actors from the Dallas area and the fiancé of one of the women" (Grigsby, 2019). This lawsuit went on for several months; at the end of it, a judge "dismissed 17 of Mignogna's claims between two separate rulings" and ruled "that [he] must pay US\$223,042.42 in attorneys' fees to the defendants [,] US $\$ 15,000$ in sanctions [to the judge, and] US $\$ 287,500$ in contingent fees [should] he [choose] to appeal" (Sherman, 2019). Judge John P. Chupp dismissed these legal 
proceedings based on the defence's argument that Mignogna had no basis for his defamation lawsuit because the defendants were covered under the Texas Citizens Participation Act (TCPA), "the state's measure to protect against SLAPP (strategic lawsuit against public participation) lawsuits" (Sherman, 2019). As well, the communications that Mignogna constructed his lawsuit with "related to a public concern, and the communications involved allegations of conduct by Mignogna that relate to health and safety, environmental, economic, or community well-being" (Sherman, 2019). Lastly, the court found that Mignogna "failed to establish, by clear and specific evidence, a prima facie case for each element of defamation, tortious interference with existing contracts, tortious interference with prospective business relations, and civil conspiracy against all defendants, as well as vicarious liability against Funimation” (Sherman, 2019). Because the defendants successfully invoked the TCPA during this lawsuit, Mignogna (the plaintiff) must pay attorneys' fees and a court-issued fine to deter him from pursuing similar legal actions. To date, Twitter users continue to add the \#KickVic and \#IStandWithVic hashtags to their tweets, and there are many instances where both hashtags are used together in the same tweet. The presence of both hashtags within the same tweet raises the question of whether \#KickVic and \#IStandWithVic always stand in exact opposition to each other. This inquiry is my guiding focus for the investigation of this study.

\subsection{Research questions}

With the goals of understanding how Twitter users discursively construct dominant ideologies of sexual harassment as well as the nature of digital activism for this specific protest and the counter-protest, this thesis asks the following questions: 
1. How do the similarities and differences of keywords and semantic themes discursively construct the protest and counter-protest?

2. How do Twitter users discursively construct women in this protest and counterprotest?

3. To what extent do these findings subvert or perpetuate (i) dominant ideologies related to sexual harassment and (ii) digital activism hashtags to address this social issue?

Because this thesis involves many factors, like theoretical concepts, methods, and data, I created sub-questions to these three questions to provide the best chance of adequately answering them. A chart detailing these questions, sub-questions, methods, and supporting theory can be found in Appendix $\mathrm{A}^{1}$.

\subsection{Research significance}

There are many reasons why this research must adopt a critical lens. Firstly, sexual harassment is a form of gender-based violence and a persistent issue in our societies. While anyone, including men, women, and people who identify as elsewhere or not at all on the spectrum of gender, can experience sexual assault and harassment, "[women] were 10 times more likely than men to be victims of a police-reported sexual assault in 2008," according to Statistics Canada's Gender Differences in Police-reported Violent Crime in Canada (Canadian Women's Foundation, 2021). On top of this,

\footnotetext{
${ }^{1}$ To examine each corpus I constructed in its entirety, I divided the first and second research questions into sub-questions where each addresses a different aspect in the corpora. By examining each corpus for its parts in conjunction with the supporting theory of the research question, it is possible to determine an answer to the third research question.
} 
Statistics Canada's Child and Youth Victims of Police-reported Violent Crime reports that " $82 \%$ of all victims of sexual assault under the age of 18 are female, and girls under age 18 report a rate of sexual violence almost five times higher than boys under 18 " (Canadian Women's Foundation, 2021). While victims do report sexual assault and harassment, there are many reasons why they do not, including fear, denial, guilt and shame, and embarrassment. As well, those in positions of authority have socialized women into roles where they are expected to “'be nice' and to behave passively in response to conflict" (Canadian Women's Foundation, 2021). This reality, coupled with the possibility that the perpetrator of the assault or harassment is in a position of authority, results in victims "[being] afraid they [will not] be believed and that the voices of men with status will be believed over theirs" (Canadian Women's Foundation, 2021). Given the power asymmetries that society perpetuates from gender ideologies where men dominate women — and therefore women are subordinate to men — a critical perspective is beneficial. The ability to examine and critique the lexical items and discursive constructions that perpetuate the overt and implicit oppressive ideologies allows for a discussion of what authority figures have come to establish as the norm and the creation of awareness for these power asymmetries.

However, this is not the only place where criticality is required. Due to these power imbalances and the efforts to change this social issue, victims of sexual abuse and harassment, as well as their allies, have started to use social media to effectively promote change. Despite this, those in positions of authority and their supporters exist on these platforms too. As such, I argue that any research on hashtags used in the service of digital activism must not only consider the discursive constructions of the protest, but also the 
counter-protest. According to a study by Mendes et al. (2018), feminists with clashing views in their offline lives found that Twitter is a "relatively safer and easier space to engage in feminist discussions than in participants' offline contexts" because they could remain anonymous (p. 243). However, researchers must acknowledge that those who oppose this creation of awareness concerning social issues also promote their ideologies on these platforms, creating "contrasting groups with different sets of opinion and beliefs who struggle against one another to reach that visibility that is so important on social media" (D'Ambrosio, 2019, p. 6). Taken together, the hashtags \#KickVic and \#IStandWithVic representing the protest and counter-protest of Mignogna's sexual harassment allegations provide an opportunity to analyze the discursive constructions related to power asymmetries of power ideologies in an instance of digital activism that only took place online.

\subsection{Researcher's standpoint}

As an advocate for raising awareness concerning social issues, who is a fan of anime and the North American voice acting industry, and who identifies as non-binary, the issues this research addresses are personal to me. In the past, I used to be a fan of Mignogna and met him once at a convention when I was a teenager. I recognize that while I have a biased perspective on this project, I view this bias as a crucial aspect that allows me to maintain a critical distance towards the situation, the findings my analysis produces, and my interpretation of these findings. Though I was not sexually harassed when I met Mignogna, this does not negate the sexual harassment allegations individuals have made to date. As someone who wants political, economic, personal, and social equality for all, I wanted women and others to be believed when they report sexual 
harassment, regardless of people's past experiences with the perpetrator. Keeping my biased perspective in mind, I hope to examine the discursive constructions of this protest and counter-protest and illustrate these findings compared to the success achieved from this instance of digital activism.

\subsection{Organization of this thesis}

This thesis is constructed of six chapters. Following the present chapter (Introduction), I begin Chapter 2 (Literature Review) by synthesizing the pre-existing literature according to themes that scholars have previously discovered in studies like this one. After reviewing the strengths and weaknesses of these studies, I discuss the concepts that serve as the theoretical lenses for this project. In Chapter 3 (Methodology), I develop my explanation of CDS to address its limitations and introduce CL, the second approach that this project uses. Furthermore, I explain how I built my three corpora and detail the procedures I use to analyze the data within them. In Chapter 4 (Findings), I present the findings of my analysis in three main parts: word frequencies, similarities and differences of semantic themes and keywords, and the discursive constructions of women. In Chapter 5 (Discussion), I interpret these findings in discussion with the theoretical concepts I detailed in Chapter 2 to answer my research questions and address the significance of this study. In Chapter 6 (Conclusion), I provide my conclusions on the research, suggest its implications, address its limitations, and discuss directions for future research. 


\section{Chapter 2: Literature Review}

In the previous chapter, I provided the research questions that ground this project, explained why this research fills an important niche and detailed the relevant background information and context for the opposing hashtags of this project. The present chapter consists of two parts that synthesize the existing literature and explores how scholars have conducted previous research on similar, overlapping topics that my research addresses. First, I illustrate how scholars have studied Twitter and hashtags, focusing on the literature that also considers hashtags in the service of digital activism. Throughout this section, I review the weaknesses and strengths of these studies, and I take this knowledge forward in my project. The second part of this chapter is the theoretical framework, where I explain the theoretical notions that serve as the lens to this study. With this information, I situate my study within this research field and determine the gap it addresses thanks to the theoretical concepts it adopts.

\subsection{Consulting the literature}

At present, Twitter is now fifteen years old, and there is no shortage of studies that analyze this social networking service and hashtags in some way or form. Therefore, I narrowed the scope from all literature scholars have published about this platform to studies focused on the relevant aspects of my research. For this study, these aspects include how authors have conceptualized hashtags, the specific hashtags that they have studied, the key themes of the discourses (tweets) that users connect to these hashtags, how tweeters use hashtags in the service of digital activism and the strategies related to this usage, and what these hashtags allowed to take place. 


\subsubsection{Recurring and relevant themes}

There are numerous studies on Twitter and hashtags, and those that heavily informed my project have at least two of the following aspects. Firstly, the study focuses on hashtags that tweeters use in the service of digital activism. Secondly, the study creates a corpus (or corpora) from tweets. Thirdly, the study researches the hashtag alongside gender issues like sexual harassment. Lastly, the study examines the key themes of the discourses from a critical perspective.

Among the studies that meet these criteria, I noticed several reoccurring research questions or focuses. These patterns occurred even when the author's (/authors') central research question(s) did not explicitly state these foci; rather, authors illustrated them in the studies' findings. One common research question of the literature examines the linguistic, discursive, and stylistic strategies of a particular hashtag or the tweets that use a hashtag. When looking at eight studies that address strategies, all written by different authors, five focus on the strategies of a specific hashtag or set of related hashtags. As well, each of these hashtags plays a role in a relevant protest of digital activism that Twitter users created them for, including \#YesAllWomen (Barker-Plummer \& BarkerPlummer, 2017), \#BlackLivesMatter (D’Ambrosio, 2019), \#BlackLivesMatter and \#AllLivesMatter (Gallagher et al., 2018), \#WhyIDidntReport (Palomino-Manjón, 2020), and \#bindersfullofwomen (Zhu, 2016). Likewise, my study examines two hashtags connected to an online protest and digital activism, \#KickVic and \#IStandWithVic, and is concerned with the unique and similar discursive constructions in these hashtags.

Another commonality within the literature that investigates the discourses of Twitter and hashtags is what the key themes were, i.e., what tweeters talked about in their 
tweets. It is beneficial to distinguish between themes, the present subject of conversation, and the topic that a hashtag represents, which I outlined in the previous chapter. Firstly, while a hashtag generally refers to one topic or discursive event, there can be many key themes within these discourses. For example, with the hashtag \#YesAllWomen, researched via content and discourse analysis methods by Barker-Plummer and BarkerPlummer (2017), the topic of the hashtag is the act of sharing stories of misogyny and violence against women. On the other hand, key themes within the discourse include terms "that [support] the description of \#YesAllWomen as a conversation about a rape culture," including "man, woman, rape, misogyny, fear, sex, violence, and feminism" (p. 16).

Two other studies that analyzed the key themes of the discourses of hashtags are D’Ambrosio (2019) and Gallagher et al. (2018). With different approaches, both studies examine similar hashtag topics; D’Ambrosio (2019) focuses on \#BlackLivesMatter, an instance of "hashtag activism in the field of racial justice," via discursive strategies and critical discourse analysis (p. 6), while Gallagher et al.'s (2018) computational study of \#BlackLivesMatter and \#AllLivesMatter discovers how this pair of protest and counterprotest hashtags diverge from each other. This line of questioning is beneficial because it helps determine what issues users are talking about in conjunction with the hashtags. As well, Barker-Plummer and Barker-Plummer (2017) compared their frequent key terms against a reference corpus (the Corpus of Contemporary English, CCAE) to determine that "these terms occurred much more frequently in \#YesAllWomen than they would in everyday discourse" (p. 16). This comparison allows for researchers to potentially pinpoint specific and salient discourses within the hashtag under investigation. Similarly, 
the analysis of this project examines the semantic themes of \#KickVic and \#IStandWithVic with the additional step of investigating whether these themes are similar or different in the opposing hashtags. Also, Barker-Plummer and Barker-Plummer's study shows that scholars can compare the key terms of a corpus against a reference corpus to investigate if these terms belong to general discourses or not.

Barker-Plummer and Barker-Plummer (2017) discovered "a very specific, political (feminist) discourse" in the hashtag \#YesAllWomen. D'Ambrosio (2019) found that tweets with the hashtag \#BlackLivesMatter included the frequent lexical items white and black, which pointed to "how the discourse pertaining to race in this case is presented as a dichotomy" (p. 12). These tweets, which create a narrative within the discourse, “also share negative judgment against police and whites and strong emotions such as fear, disappointment and exasperation" (p. 18). Though D’Ambrosio does include counterprotests in the analysis of \#BlackLivesMatter, an element that I believe should have been included, Gallagher et al. (2018) directly compare \#BlackLivesMatter and \#AllLivesMatter. This study finds that the hashtag \#AllLivesMatter, which at first glance should encompass a discourse where all lives matter, reveals "that the only other lives that are significantly discussed within [\#AllLivesMatter] are the lives of law enforcement officers, particularly during times in which there is heavy protesting" (p. 18). While Gallagher et al. (2018) does not investigate these hashtags from the perspective of critical discourse studies, I argue that they identified discourse within \#AllLivesMatter. In this hashtag, this discourse is pro-law enforcement officers and "often frames Black protesters versus law enforcement with an ['Us versus Them'] mentality" (p. 18). From van Dijk (1998), this discursive construction involves the four moves of the "ideological square", 
where each move can belong to a different corner of the theoretical and ideological square (p. 267). Firstly, the positive aspects of Us are emphasized while the negative aspects of Them are emphasized. Secondly, the positive aspects of Them are suppressed while the negative aspects of Us are suppressed. Together, these moves allow the speaker/writer to position other actors against them and, at the same time, construct these other actors negatively. Through the quantitative and qualitative analysis of \#KickVic and \#IStandWithVic, I intend to discover unique constructions in the discourses of the opposing hashtags.

Of particular interest to many studies, including the present one, is how individuals use hashtags in the service of digital activism. Several findings are reported through this line of questioning since the function and context of the hashtag influence how tweeters use it. For example, in the case of advocacy organizations, Saxton et al. (2015) discover prevalent usage of hashtags for topics like medical conditions, health goals, police advocacy, health-related events, and conferences, as studied with a twostage mixed-methods approach involving inductive analysis and regressions.

Additionally, Saxton et al. (2015) highlight that hashtags meant to educate the public on medical conditions, health-related research, and health-related public policy were the most frequent in their data. Lastly, through their analysis, they identify three distinctive strategies of how advocacy organizations use hashtags: to increase interaction through retweets, "to find common ground with partners across sectors," and to use specific hashtags to catch the attention of their audiences (p. 19).

However, these are not the only strategies of hashtags that have been identified, especially considering those studies that focus on one or two hashtags for digital 
activism. One such discursive strategy (relating to discourse) is indexing, where a hashtag creates a meaningful connection between a tweet and the social issue that the hashtag references (Bonilla \& Rosa, 2015). For example, tweeting about the action of 'holding car keys like a weapon when walking at night' along with the hashtag \#YesAllWomen makes the connection between that it is terrifying as a woman to be living in a rape culture and emphasizes the social issue that women are constantly living in a rape culture (Barker-Plummer \& Barker-Plummer, 2017).

Another strategy that Gallagher et al. (2018) discover is that of hashtag hijacking, "where one group adopts the hashtag of [a] politically opposed group in order to inject their ideological beliefs" (p. 18). While the advocates of the politically oppositional stance created the hashtag \#AllLivesMatter, Gallagher et al. (2018) find that \#BlackLivesMatter advocates were the reason for a significant portion of the hijacking that occurred in the original protest hashtag. By using the protest and counter-protest hashtags together, the authors argue that \#BlackLivesMatter advocates were able "to directly interrogate the stance of 'All Lives Matter' and the worldview implied by that phrase" (p. 18). Despite expressed concerns that the counter-protest hashtag would derail the movement of the original protest hashtag, this finding shows that \#BlackLivesMatter has found a way to counter the counter-protest.

Barker-Plummer and Barker-Plummer (2017) find another instance of hijacking in their study of \#YesAllWomen, though for very different reasons. Due to the inadequate intersectionality of the hashtag, "some trans women, gay women, and women of [colour]" created parallel hashtags like \#EachEveryWoman and \#YesAllWhiteWomen to both better represent them and "critique $[\ldots]$ the 'white feminism' they came to see as 
dominating the hashtag" (p. 24). Like Gallagher et al.'s (2018) study, Barker-Plummer and Barker-Plummer (2017) also discover that tweets with \#YesAllWomen were crosstagged with politically opposite hashtags like \#NotAllMen, \#YesAllMen, and \#YesAllPeople. They suggest that the oppositional stances and "'internal' feminist critique" indicate that "the conversation about rape culture was taking place on multiple fronts at the same time" (p. 26). Given how previous studies have identified strategies of hijacking in opposing and related hashtags that individuals use in the service of digital activism, I expect to discover similar findings in my research because of the hashtags that this thesis examines.

Lastly, many studies find that hashtags allow users to share personal accounts, experiences, and micro-stories to subvert dominant ideologies (Clark, 2016; D’Ambrosio, 2019). These accounts, charged with strong emotions, bring a highly personal type of discourse to the space of a protest hashtag and "invite[s] the ambient audience to evaluate" the event with a similar stance (D'Ambrosio, 2019). These personal experiences also highlight social inequalities that marginalized groups face, providing evidence that hashtags are an important and effective way for these groups to advocate for change. Clark (2016), in an exploration of hashtag feminism's features and significance, discovers a similar finding in her study of \#WhyIStayed. In this study, survivors use the hashtag as a narrative story prompt "to frame their diverse experiences in a compelling manner in 140 characters or less" (p. 796). Using this hashtag, victims took Beverly Gooden's (the creator of \#WhyIStayed) originally "dark parody of mainstream media discourse" and through their accounts, answered "mainstream media's problematic question for victims [:] why did you stay with your abuser?” (p. 796). 
Because the current project studies hashtags that allow users to share their experiences concerning Mignogna, both those good and bad, I intend to discover how users subvert or perpetuate power asymmetries of gender ideologies in their tweets and which hashtag(s) they use to distribute these ideologies.

\subsection{Theoretical considerations}

Having reviewed the recurring and relevant themes from previous studies that have analyzed hashtags used in the service of digital activism, I discuss in this section the theoretical elements I use to conceptualize this phenomenon in my research. As realized from the following review, there is no study to my knowledge that critically analyzes the discourses of a protest and counter-protest through multiple corpora alongside the concepts I draw on in this study. Focusing on the idea that hashtags are a digital space that allows Twitter users to commune together around shared ideas, I begin this section with a discussion of affinity spaces (Gee, 2005) and ambient affiliation (Zappavigna, 2011). Additionally, I explain what digital activism is, including its importance and relation to affinity spaces. Lastly, because these hashtags confront a social issue, I find it relevant to use critical discourse studies (CDS) and feminist critical discourse analysis (FCDA) as a crucial theoretical lens to understand this phenomenon.

\subsubsection{Affinity spaces and ambient affiliation}

One alternative to a "community of practice" (Lave and Wenger 1991; Wenger 1998, as cited in Gee, 2005) that Gee (2005) considers is the notion of, as he calls it, an "affinity space" (p. 214). While the former focuses on "membership in a community," the latter is more interested in "the idea of a space in which people interact" (p. 214). Some aspects of the notion of a "community of practice" cannot account for how people come 
together on Twitter. For example, “a 'community' can carry connotations of 'belonging' and close-knit personal ties among people which" on the microblogging service do not necessarily exist (p. 214). It is important to remember that users do not need to know each other to collaboratively use a hashtag in the service of digital activism. Therefore, there must be another purpose that brings these individuals together that does not rely on connections with others.

Another strong notion of "community" is membership, where the people in the community are "members." However, as Gee (2005) points out, membership is not a status that can be easily defined because it "means such different things across different sorts of communities of practice, and there are so many different ways and degrees of being a member in some communities of practice that it is not clear that membership is a truly helpful notion" (p. 214). Of course, every account is a member of Twitter, but what does it mean to be a member of a hashtag and how is this membership established? It is not clear if a Twitter user would only need to use a hashtag once to be a member, multiple times, or at least every day. As well, Gee (2005) notes that while efforts have been made to distinguish a "community of practice" from other social groupings, others have nonetheless used the notion "to cover such a wide array of social forms" (p. 214). This issue makes the notion difficult to use, and I argue this is especially the case with social media, as a researcher cannot be sure how their audience may or may not understand what a "community of practice" is.

With these issues considered, Gee (2005) states that the key problem with the notion of a "community of practice" is that it always attempts "to label a group of people" (p. 215). When a label exists, there immediately will be those individuals who 
fall under the label, those that the label partially includes, and those who do not meet its definitions. This label defines the community and discussing a community is halted "until we have defined who is in and who is not, since otherwise we cannot identify the community" (p. 215). However, as briefly discussed in the above examples, participation, membership, and boundaries related to the label of a community are not straightforward to define.

For these reasons, Gee (2005) wants to switch the focus of social grouping from those who would belong to it, or identify as members, to the space that the group encapsulates. He calls these spaces "semiotic social spaces" ("SSS" for singular, "SSSs" for plural) because the draw of "these spaces is [how] people get and give meanings to signs within them" (p. 216). These spaces are not just physical or geographical as people also interact with others around topics in virtual spaces. An SSS has various parts that make it work. First, Gee (2005) says, is that it needs to be "about" something or have some content because it is not just any social space but a semiotic social space (p. 218). For SSSs, this content is called a generator as it generates "the signs that make up the content of the space" (p. 218). In the context of my study, both the hashtags \#KickVic and \#IStandWithVic are generators because there exists "a characteristic set of multimodal signs to which [they] can give specific sorts of meanings and with which they can interact in various ways," like a hashtag (p. 218). A generator follows a set of signs, which can be examined both internally and externally. When viewing an SSS internally, this involves "a set of signs [or a type of content]" (Gee, 2005). Likewise, looking externally at an SSS means to view "the individual and social practices in which people engage in respect to the set of signs" (p. 218). 
If we look at these aspects from the perspective of Twitter, the internal lens shows that users can tweet and add resources to their tweets to enhance meaning-making, like hashtags, pictures, videos, and hyperlinks. From the external perspective, people use Twitter in real-time and interact with other users on the platform in various ways. As well, users are aware that, in terms of how they use Twitter, not everybody on the platform uses it in the same ways. Following Gee's (2005) logic, an internal view of this SSS means to ask about its design, whereas an external view means "to ask about [how] people [organize] their thoughts, beliefs, values, actions and social interactions in relation to the signs made available [on the platform]" (p. 219). These views and how we look at them are also classified as a "grammar", a term that Gee (2005) refers to as "phenomena that are emergent" (p. 219). The internal design of Twitter comes from the designers of the microblogging service, and this creation 'interacts with players' desires, skills, actions and interactions" (p. 219). This design feeds into the external grammar of Twitter, as the signs that are a part of the platform's design organizes users' "thoughts, deeds and interactions" and these are realized through users' "actions and interactions as these begin to take on some (however loose) regularity or patterning” (p. 219).

The final component required to understand an SSS is the concept of portals, or how people gain access to the SSS, as well as its signs and "ways of interacting with those signs" (Gee, 2005, p. 220). For example, the desktop website is a portal for Twitter, and so is the smartphone application that users can download on their devices. Twitter via Short Message Service (SMS), a service where users send tweets and "receive SMS messages containing the text of new tweets," was also a portal (Kastrenakes, 2020). Even though this service is now only available in a few countries (Twitter Support, 2020), this 
was once a popular portal for mobile users, especially when Twitter was founded in 2006 (Kan, 2020). Other portals for Twitter include TweetDeck (a dashboard application to manage Twitter accounts), when online news articles embed tweets in their stories, and when people share a tweet on other social media platforms. As Gee (2005) elaborates, a portal gives access to an SSS but is only a "subspace" of the larger space, or a portion of the whole (p. 220). This feature is true of hashtags used in the service of digital activism too. For example, the issues related to sexual harassment that \#KickVic addresses are a part of the advocacy movement of \#MeToo.

From Gee's conceptualization, I argue that hashtags, especially those that tweeters use in the service of digital activism, are both SSSs and portals. Hashtags can be both because, as Gee (2005) states, portals allow individuals "to interact with the signs generators have generated," and portals can potentially "become generators [...] if they allow people to add to the signs or change the relationships of the signs other generators have generated" (pp. 220-221). Similarly, "a generator can also be a portal" if it provides signs and content and gives access to the SSS to interact with those signs and content ( $\mathrm{p}$. 221). Applying this logic to hashtags related to digital activism, \#KickVic is a sign on Twitter (the generator) because users have given it a specific meaning and use it to interact with others. Overall, the purpose of the SSS as a notion instead of a "community" is to let researchers inquire "about what thoughts, values, actions [,] and interactions go on in this space, by whom and with whom [,]" without assumptions of membership involved (p. 223). I provide a diagram of an SSS below in Table 1; text in square brackets defines the cell found above it. 
Table 1: Social semiotic space (Gee, 2005, p. 221)

Generator(s)

\begin{tabular}{c|c}
\hline \multicolumn{2}{c}{ [gives us a set of signs and possible relations among them] } \\
\hline Internal Grammar & External Grammar \\
\hline [design of content] & $\begin{array}{c}\text { [patterns in thoughts, deeds, and } \\
\text { interactions] }\end{array}$ \\
\hline \multicolumn{2}{c}{ Portal(s) }
\end{tabular}

[offers access to signs and interactions with them]

While many types of SSSs exist, Gee (2005) is concerned with a particular type that he calls an "affinity space" (p. 216). He argues that affinity spaces "capture one characteristically modern and important form of social affiliation," which is that they are “where effective learning occurs" (p. 217; p. 231). Gee's (2005) notion of an affinity space can be defined by eleven features. However, if an SSS does not have all these features, it does not mean that we cannot consider it an affinity space. Rather, Gee (2005) argues that we should consider affinity spaces on a sliding scale, where "any SSS that has more of these features than another is more of an affinity space than the other or is closer to being a paradigmatic affinity space” (p. 225). These features (directly from Gee, 2005) are:

1. Common endeavour, not race, class, gender or disability, is primary

2. Newbies [newcomers] and masters and everyone else share common space

3. Some portals are strong generators

4. Internal grammar is transformed by external grammar

5. Encourages intensive [specialized] and extensive [general] knowledge

6. Encourages individual and distributed knowledge

7. Encourages dispersed knowledge

8. Uses and [honours] tacit knowledge 
9. Many different forms and routes to participation

10. Lots of different routes to status

11. Leadership is porous and leaders are resources

Gee (2005) argues that affinity spaces are abundant in "our global high-tech new capitalist world" (Gee 2000, 2001; Rifkin 2000, as cited in Gee, 2005, p. 228). Examples include businesses organizing affinity spaces for their customers, scientists across disciplines interacting with other individuals and groups in networks that resemble these spaces, and fans of media creating affinity spaces to engage with other fans. Gee (2005) mentions social activists in his list of affinity spaces that are common today. Regardless of their cause or the social issue they work to change, the affinity spaces that social activists create make it possible for "people who may share little, and even differ dramatically on other issues, [to] affiliate around their common cause and the practices associated with espousing it” (p. 229). According to Gee (2005), the affinity spaces social activists use to affiliate "have most or all of" the eleven features in the list above (p. 229). Although he does not describe which of the eleven features these specific affinity spaces may not have, I expect the hashtags that people use in the service of digital activism would pattern similarly.

But what is affiliation, and how does it happen in tweets? Zappavigna's (2011) discussion on 'folksonomy' (Vander Wal, 2007), collaborative tagging, and her notion of ambient affiliation help to answer this question. Because Twitter constrains how many characters are in a tweet, these environments produce "interesting cases in making meaning" (p. 790). Also, even though a user posts a tweet, "there is no communal expectation that anyone respond to [it]" (p. 790). However, due to the "social need 
among users to engage with other voices," we observe tweeters creatively using what Gee (2005) refers to as a sign to engage with others on the platform around common topics.

As Zappavigna (2011) notes, this creative usage of signs (in her words, punctuation which equates to typographic meaning potential) helps this affinity-driven "movement toward Twitter becoming a form of "public conversation"” (p. 790). The qualities of this 'public conversation' include that it involves more than two parties (multiparty), it is not permanent or stagnant (temporarily fluid), and there is a strong relationship between texts (highly intertextual). One such linguistic marker or sign that has evolved on Twitter is the hashtag, which has many functions, including marking "the topic of a tweet" (p. 791). On top of this, hashtags also serve the purpose of metadata, in that it gives us information and describes other data (the text of the tweet). According to Zappavigna (2011), linguistic markers (hashtags) exist within tweets to encourage heteroglossia (Bakhtin, 1981, as cited in Zappavigna, 2011). On Twitter, heteroglossia takes the form of these markers and brings "other voices into tweets by addressing other users, republishing other tweets, and flagging topics that may be adopted by multiple users" (p. 790). A hashtag, which is also a linguistic marker, is construed a bit differently in that "it presupposes a virtual community of interested listeners who are actively following this keyword or who may use it as a search term" (p. 791). Recalling Gee's (2005) discussion, this virtual community that a hashtag presupposes is known as an SSS and/or an affinity space.

One aspect of tweets with hashtags that are important for understanding the remainder of Zappavigna's (2011) discussion is that when a tweeter uses a hashtag, it creates "an attributive relationship between the tweet as a tagged token and the tag as its 
type" (p. 791). Explained simply, the tweet is a tagged token, and the hashtag describes what type this token is. This notion of an attributive relational process comes from Halliday and Matthiessen (2004, as cited in Zappavigna, 2011), where they define this relationship as "an entity [that] has some class ascribed or attributed to it" (p. 219). Additionally, a tweeter assigns a hashtag the role of a keyword in their tweet, and this keyword is "metadata referencing the topic of the message" (Zappavigna, 2011, p. 791).

Moreover, the hashtag representing a type of tweet assumes that other people will also use this hashtag to represent a keyword for their tweets on the same topic. These generated keywords that describe a topic (and for the case of digital activism, a social issue) provide what Gee (2005) calls a portal "into the social realm of collaborative tagging, or 'folksonomy"' (Vander Wal, 2007, as cited in Zappavigna, 2011, p. 791). Folksonomy, as Vander Wal (2007) describes, is the product of people freely tagging information and objects so they can retrieve them later. People create these user-defined tags and use them in what Gee (2005) calls an SSS (Vander Wal, 2007 refers to it as a social environment). Considering all of this, I view hashtags as signs, generators, and portals, where the lexical items and the semantic meanings of a hashtag represent an affinity space. This relationship creates a folksonomy because of the collaborative tagging practices that are occurring in the space. With this stated, I find it crucial to note that while users engage in collaborative tagging, which creates portals to an affinity space, this does not mean every user shares the same values as others, especially in the context of a protest with a counter-protest. I want to emphasize that portals are not gates in that they allow any user into the affinity space; though a hashtag might encourage those tweeters who share values with others, this is not a requirement for engagement. 
As previously stated, hashtags are a sign that representing the type of a tweet through the existence of a keyword. Because a hashtag is a searchable Twitter portal, using this convention in a tweet increases the possibility that other Twitter users will find it, and this keyword makes a user's tweet more noticeable. These features lead to the possibility that more people may follow this user, and at the same time, a larger audience will read their tweets (text). To affiliate ambiently means to "affiliate with a copresent, impermanent [space] by bonding around evolving topics of interest" (Zappavigna, 2011, p. 800). Though Gee (2005) uses the term affinity space to refer to space where the bonding happens, both his term and Zappavigna's ambient affiliation are closely related. Zappavigna (2011) highlights that these evolving topics that users can bond around are a part of Twitter's design in the form of 'trending topics', which are "a list of keywords occurring with high frequency in current posts, displayed under the search box" (pp. 800801). The tweet's 'loudness' is strengthened when a hashtag reaches trending status, giving the hashtag "an additional semiotic pull that may be likened to a gravitational field" (p. 801). Taken together, a hashtag labels an affinity space of discourse that it encompasses because it is a sign and makes the values of the tweet searchable, allowing others to enter the affinity space and affiliate ambiently.

Lastly, Zappavigna (2011) argues that the increased meaning potential for hashtags "is both a product of the reduced affordances of the character-constrained mode and part of a 'multiplication' of what it means to talk online" (Zappavigna, 2011, p. 801). The hashtag has both a searchable function and a social function. First, it allows users to retrieve the online discourses of an affinity space. Secondly, it simultaneously groups tweets together and allows the creators and audience of those tweets to interact within the 
space. Similar to what Gee (2005) says in respect to the affinity spaces of social activists, Zappavigna's (2011) affiliation is ambient because "the users may not have interacted directly and likely do not know [each other and] may not interact again" (p. 801). To consider all of this-SSSs, affinity spaces and their features, folksonomy, couplings, and ambient affiliation — as they relate to hashtags that tweeters use in the service of digital activism is no easy task. However, when addressing the social issue of sexual harassment within the North American voice acting industry and anime convention sphere, I argue that this hashtag for digital activism was the most helpful tool in this instance. In my view, \#KickVic's functions as they relate to the above concepts are as follows. First, it is a searchable keyword, a topic marker for the issue, and a sign within the SSS of Twitter that has a specific meaning that allows users to interact. Additionally, \#KickVic is a portal into the SSS about sexual harassment allegations. Furthermore, this hashtag is an affinity space where users affiliate ambiently through shared values that allow them to come together under a cause and support it.

Furthermore, \#KickVic is the beginning of a folksonomy as from this usergenerated hashtag tweeters created several other related hashtags (ex. \#IStandWithVic, $\# K V$, \#ISWV) within this social environment. Altogether, this folksonomy creates a complex network of searchable keywords and relevant topics, serving as signs within various SSSs and portals in the discourses. Given this folksonomy of related hashtags that yet have different personal evaluations of the discourses attached to them, this ambient affiliative network becomes an interesting example of digital activism on Twitter. It is for this reason, as well as my relationship to the affinity space of online anime subculture, that I believe this instance of people using hashtags in the service of digital activism will 
add new insights to the larger conversation of how activists talk about the social issue of sexual harassment online and how those users in political opposition to them argue against their advocacy.

\subsubsection{Digital activism}

According to Gee (2005), social activists create affinity spaces, allowing many individuals from various backgrounds to affiliate around a topic with the greater purpose of enacting social change. For the remainder of this paper, I will refer to these affinity spaces as digital protests. As well, I call the result of these protests digital activism. According to Bonilla and Rosa (2015), a digital protest feels like an in-person protest because of the "shared temporality," or rather than merely reading about an event, individuals can participate in real-time with it as it unfolds (p. 7). Discussing the context of \#Ferguson, Twitter users watched live streams of the horrific treatment of journalists and protestors in Ferguson, and through this activity, they felt like they too were participating in a protest. What makes these protests digital is that they occur in online spaces, like Twitter, and potentially connect more individuals than ever could be possible at an in-person protest.

Of course, one element of any protest is the element of activism. With the meaning of "triggering sociopolitical change with $[\ldots]$ the help of collective action," activism is the goal for any protest advocating against a social issue (Clark, 2016, p. 791). As such, digital activism is also activism with the added element that it occurs online. While some have called into question whether activism that occurs digitally to be "real" (Wasik, 2009, as cited in Bonilla \& Rosa, 2015; Gladwell, 2010, as cited in BarkerPlummer \& Barker-Plummer, 2017), the reasons why this activism exists dispels this 
concern. Firstly, Mendes et al. (2018) found that feminists with conflicting perspectives in their offline lives believed that Twitter is a "relatively safer and easier space to engage in feminist discussions than in participants' offline contexts" because they could remain anonymous (p. 243). Additionally, these digital protests at times critique and create awareness about matters like police practices and media representations, and as such protestors cannot rely on these systems to help or share their message for worry that these challenged systems would harm them (Bonilla \& Rosa, 2015). With regards to feminism, Clark (2016) notes that feminists of colour and those that work outside of formal organizations use digital activism because it fosters an inclusive space for "diverse voices of protests" (p. 790). Unlike "the highly structured, well-resourced, and predominantly white, middle-class organizations that became focal points for feminism during the 1960s and 1970s," affinity spaces like hashtags, which are also portals to an SSS, allow a wide audience to access and participate in the movement, acting as space where "protest can turn into collective action" (p. 790). Since digital activism turns protest that critiques social issues into collective action to create change, it is necessary to analyze the discourses of this activism through a critical lens.

\subsubsection{An overview of critical discourse studies}

Critical discourse studies (CDS)—referred to as Critical Discourse Analysis (CDA) in the past - is a group of approaches that seeks to "advance our understanding of how discourse figures in social processes, social structures and social change" (Flowerdew \& Richardson, 2017, p. 1). Similarly, Feminist Critical Discourse Analysis (FCDA) shares goals of CDS, with the same focus on "the complex workings of power and ideology in discourse" and the addition of "[advancing] a rich and nuanced 
understanding of [how the above is sustained] in [...] hierarchically gendered social arrangements" (Lazar, 2007, p. 141). To understand the approaches of CDS, including FCDA, and their importance, it is crucial to understand what practitioners mean by the terms discourse, ideology, power, identity, and context. For CDS approaches and FCDA, the term discourse embodies text, talk, conversations, documents, and much more. In other words, discourse is language in use. With this said, practitioners also use discourse "to refer to a specific set of meanings expressed through forms and uses which give expression to particular institutions or [groups]" (Flowerdew \& Richardson, 2017, p. 2). Used according to this definition, it is possible to talk about specific discourses, like feminist discourse and gendered discourse (Lazar, 2007). What makes a specific discourse recognizable is "the ideologies they convey and the linguistic and other semiotic structures through which they are expressed" (Flowerdew \& Richardson, 2017, pp. 2-3). Semiotic systems that realize the structures of the discourse include not only language but also those that are "visual and aural (multi-modal) systems of signs" (p. 3). This aspect is very important for those practitioners who use CDS approaches to analyze the discourses of the internet, social media included, as multi-modality is abundant online.

Hinted above during the discussion of specific discourses is the term ideology, "which are sets of beliefs and values belonging to particular social groups" (Flowerdew \& Richardson, 2017, p. 3). Though Destutt de Tracy, an early 1800s French philosopher, coined this term, it is commonly associated with Karl Marx who had a slightly cruder interpretation (Machin \& Mayr, 2012; Flowerdew \& Richardson, 2017). According to Lazar (2007), Marxist accounts developed a view of ideology to be "representations of 
practices formed from particular perspectives in the interest of maintaining unequal power relations and dominance" (p. 146). Though they developed this definition regarding class relations, Fairclough \& Wodak (1997, as cited in Lazar, 2007) note that it can account for "other relations of domination, including gender" (p. 146). An example of an ideological structure - how an ideology is represented in the practice of society - as related to FCDA is the predominant notion of gender. This structure, according to Lazar (2007), "divides people into two classes, men and women, based on a hierarchical relation of domination and subordination, respectively," and this structure "imposes a social dichotomy of labour and human traits on women and men" (p. 146).

When discussing ideology, I must clarify what practitioners mean by legitimation and power. These notions relate to an ideology's influence, or how it is dissipated throughout society. The process of legitimation (Hodge and Kress 1993; van Leeuwen 2008, as cited in Flowerdew \& Richardson, 2017) for an ideology is that it "[comes] to be accepted as part of common sense" (p. 3). In other words, legitimation is "a set of beliefs and values [that] becomes accepted by virtue of the fact that society accepts the authority of those disseminating them" (Flowerdew \& Richardson, 2017, p. 3). From my perspective, the dimension of power is like that of legitimation because they are often both held, disseminated, and exercised by those individuals within a society who have authority. According to Foucault (1982, as cited in Flowerdew \& Richardson, 2017), CDS practitioners see that power is everywhere in society and that those in authoritative or privileged positions can maintain their positions "through their exercise of power" (p. 4). The theory of hegemony from Gramsci (1971, as cited in Flowerdew \& Richardson, 2017) explains that elites exercise their power "not just through physical coercion, but 
covertly, through ideology and discourse" (p. 4). Lazar (2007) repeats this sentiment that power is not always a physical overt action. She views power relations as "a struggle over interests" where these interests "are exercised, reflected, maintained, and resisted through a variety of modalities, extents, and degrees of explicitness" (p. 148). Ideology and power are intertwined because privileged individuals reinforce the ideologies that construct the discourses that build our societies, to the extent that all members of society view their ideologies as common sense. This reinforcement and construction of power often happen implicitly through language, and therefore CDS strives to unravel this complex relationship between discourse, ideology, and power to enact social change or understand how members of society enact this change.

Viewing this relationship under the lens of FCDA, practitioners are concerned "with critiquing discourse which sustain a patriarchal social order" (Lazar, 2007, p. 145). In other words, due to the power men as a group have had throughout history, FCDA examines how their discourses have embedded their ideologies into society, placing them into systematically privileged positions in society while the same system “disadvantage[s], exclude[s], and disempower[s] women as a social group" (Lazar, 2007, p. 145). Overt and traditional examples of gender asymmetry or sexism include “exclusionary gate-keeping social practices, physical violence against women, and sexual harassment and denigration of women" (p. 148). However, Lazar (2007) remarks that these power asymmetries and the gender ideologies behind them have become more subtle in late modern societies in the form of power that is discursive. Though overt realizations of power still exist within society, FCDA is also consciously aware of these subtle discursive manifestations through language, as well as how Foucault's, 
Bourdieu's, and Gramsci's (as cited in Lazar, 2007) “relations of power and dominance [...] can be discursively resisted and counter-resisted in a dynamic struggle" (p. 148). As such, a study using FCDA should task itself with examining "how power and dominance are discursively produced and/or (counter-)resisted in a variety of ways through textual representations of gendered social practices, and through interactional strategies of talk" (p. 149).

For my project, I take up the above task by questioning to what extent Twitter users subvert or perpetuate oppressive patriarchal ideologies through discursive constructions of gendered power asymmetries. Users index the discursive constructions of these power asymmetries through hashtags (textual representations), creating an affinity space with multiple portals for this protest and counter-protest that are built on the subversion and perpetuation of ideologies. Altogether, the index of these discursive constructions within this space fosters digital activism, where tweeters who access the affinity space through the hashtags both resist and counter the address for social change surrounding the issue of sexual harassment.

Another important concept for CDS is identity, or how a person or a group views and situates themselves with others through various qualities, beliefs, looks, expressions, and personality. Identity is a construct that can change over time and space and is demonstrated not only through social practice but also discursive practice. Lazar (2007) notes that gender is one social category of social identity that also intersects with other categories like "sexuality, ethnicity, age, (dis)ability, social class and position, and geographical location" (p. 141). This intersection of gender with other categories with social identity makes it problematic to talk "of 'women' and 'men' in universal, totalizing 
terms" (p. 141). As such, one focus of FCDA is to show the complex ways that gendered assumptions (not strictly those that only separate 'women' from 'men'), as well as power and dominance relations, are discursively constructed, maintained, negotiated, and challenged in various contexts.

A discussion of discourse and CDS cannot occur without the notion of context. People use language and produce language from other contexts, and as such, all instances of language are embedded within their relevant context(s). Context includes all elements of the social, economic, and cultural world and its relationship to the text (I use this word to refer to an instance of language) can be immediate or broad. Depending on the view of CDS that is adopted (Fairclough, 1992; Wodak, 2014; van Dijk, 2008) the view of context within the approach will change. Fairclough's approach to discourse analysis (which context is a part of) "identifies three integrated levels" that each subsumes or constrains the previous: "analysis of text, analysis of discourse practices (production, distribution and consumption), and analysis of social practices" (Flowerdew \& Richardson, 2017, p. 5). The text is the micro-level, the discourse practices are the mesolevel, and social practice (social conditions) is the macro-level that "[involves] the role of power" (p. 5). Although this appears to be a one-way model, the relationships between the levels "are interactive, involving a continual shunting between the micro-analysis of texts, the macro-analysis of social structures and formations and power relations and the mediating role of discursive practices and discourse processes" (Richardson 2007, as cited in Flowerdew \& Richardson, 2017, p. 5). On the other hand, Wodak's (2014, as cited in Flowerdew \& Richardson, 2017) approach "identifies four levels of contextual analysis: the immediate co-text [,] the other texts and discourses that the text draws upon 
[,] the conditions of text production, distribution [,] and reception [, and] the wider sociopolitical formation" (p. 5). Finally, there is van Dijk's (2008) socio-cognitive approach, which is concerned with "mental processes involved in how we relate to people in our social world" (Flowerdew \& Richardson, 2017, p. 6). This processing of the social world constructs mental models that "represent the mediating link between text and context, between text and social structure" (p. 6). Because language obtains its meaning in context, and because it is through language that those individuals in privileged positions enact power asymmetries to spread dominant ideologies and maintain the status quo, it is crucial to analyze discourses that work to dismantle these ideologies within the context that users create them.

The final feature of CDS, as the name implies, is that the work is critical. This critique is aimed not only outwards at discourses under analysis but also inwards at the self (more on this in the next section). Critique is unavoidable in work that challenges societal power imbalances and how they are (re)produced in discourse. Rather than giving negative judgements, the work of CDS should serve "a positive emancipatory function" (p. 5). In adopting this critical lens, CDS research adopts a specific agenda with the goal of either creating social change or supporting those individuals that struggle with the inequality under investigation.

\subsubsection{Feminist critical discourse analysis}

Having explained the core theoretical notions of CDS - as well as FCDA's conceptualization of them - I find it appropriate to address the six principles of FCDA (where five are interrelated) as Lazar (2005, 2014, as cited in Lazar, 2017) has identified them. One important aspect to remember is that feminist studies, and not strictly those 
that we would call FCDA, "had provided an impetus in the formative years of CDA scholarship in the 1980s (van Dijk, 1991)" (Lazar, 2017, p. 372). This is because both CDA and feminist studies are known for their "overtly political stance and [are] concerned with [the] analysis of various forms of social inequality and injustice" (Lazar, 2007, p. 142). Though feminist studies, as well as feminist approaches in women's studies, gave momentum to CDA, the feminist linguistics of the time did not feel it necessary to explicitly label their work as feminist CDA as they were "working quite happily under the rubric of CDA" (p. 143). It was not until decades later that the term FCDA was introduced; in contrast to the past, feminist linguistics now explicitly signal "the ongoing contributions of feminist thought and politics in gender-related CDA studies, as well as the disciplinary hybridity which that has entailed" (Lazar, 2017, p. 372).

The first principle of FCDA is "the ideological character of 'gender"” (Lazar, 2017, p. 373). For FCDA, gender is both an ideological structure and practice, meaning that it is a set of values, beliefs, and ideas within society and that individuals practice these values, beliefs, and ideas in this space. Although I, along with contemporary theories, agree that gender in our world is much more fluid than a fixed binary with men on one side and women on the other, Lazar (2017) notes that this binary, as well as the stereotypes of it, is what "predominates in common sense understanding" (p. 373). While gender is complex and is just one part "a matrix of other socially stratified identities" and is not "materially experienced nor discursively constructed in the same way for all women and men," it is the ideological structure of gender that people (re)produce everyday within institutions and everyday practice (p. 373). 
As explained in the previous section, FCDA's second principle is that the notion of power is of the utmost importance for "critical investigations of gender identities and relations" (p. 373). For FCDA, there exist two broad sources of power: the concept of the 'patriarchy' for earlier feminist theories and Foucault's (1977, as cited in Lazar, 2017) concept of power which is much more subtle and explains power relations of our modern day.

The third principle regards how FCDA views discourse, in that this approach sees "[t]he relationship between discourse and the social is a dialectical one, in which discourse constitutes and is constituted by social practices" (Fairclough 1992, as cited in Lazar, 2017, p. 373). Explained through the structure of gender, individuals' discourse creates the social practices associated with gender identities and at the same time the social practices associated with gender identities shape the discourse of individuals. Because of this, the concept of identity is crucial for FCDA as it "aims to investigate how gender plays out in these identity matrices - as more or less relevant or salient - in given situations, as well as in the gendering of [other identities]" (Lazar, 2017, p. 374).

One aspect of FCDA and CDS, as the name says, is the practice of critical reflexivity. This is the fourth principle of FCDA, specifically concerning how social actors in our modern world harness this critical awareness and reflexivity. There are many ways that social actors can use this reflexivity, of which one is "for critically aware persons and institutions to generate constructive dialogue and/or spearhead progressive social changes" (p. 374). This includes speaking out, creating awareness, and holding individuals accountable for actions like sexual harassment. No one is exempt from critical self-reflexivity, including feminists, as it is crucial that while advocating for 
social justice, we do not repeat "patterns of privilege and exclusion, or bar progressive social change" (p. 374).

In feminists' aim for social justice, so too does FCDA scholarship constitute analytical activism. This fifth principle of FCDA draws upon all principles discussed previously. By advocating for a society where gender — an ideological structure and character-does not inherently decide one's identity and relationships, feminists need to facilitate "ways of 'doing' and 'becoming' that are socially inclusive and respectful of all persons" (p. 374). Through FCDA scholars' work of "voicing critique" (hooks, 1984, as cited in Lazar, 2017, p.374), communities of resistance and activism can form. On top of this, the creation of critical awareness that occurs in their work is a form of feminist activism, where "the political critique of discourse can be viewed not only for action, but as action" (p. 374).

The final principle of FCDA that Lazar (2017) discusses is that the dimension of transnationalism is imperative for this type of research. Because gender struggles vary across the globe, it is important to remember the context of the analysis. For example, gender ideologies may function in different ways, and due to this, people will use appropriate (within that context) discursive strategies to respond to them. Lazar suggests that such transnational research could provide findings of local discourse strategies that might resonate on a global scale too.

\subsection{Summary}

In this chapter, I have investigated the relevant literature to situate and inform my research project. Firstly, I discussed recurring and relevant themes scholars have previously discovered concerning hashtags used in the service of digital activism. During 
this, I considered the perspectives, approaches, and methods of these studies, including those that constructed corpora for hashtags of digital protests. This review allowed me to learn how they have studied these hashtags and pinpoint a niche that my project can address. Lastly, I discussed the theoretical framework for this study, which included affinity spaces and ambient affiliation, digital activism, CDS, and FCDA. The next chapter, methodology, explains what corpus linguistics is and how I used the synergy between corpus linguistics (CL) and CDS/FCDA to analyze the discourses of this digital protest with the corpora I constructed. 


\section{Chapter 3: Methodology}

Up to this point, I have investigated previous studies with a critical eye to understand how they analyzed hashtags used in the service of digital activism and how these findings allowed them to understand the discursive constructions within these hashtags. The results of the literature review showed that many of these studies use corpus linguistics (CL), feminist critical discourse analysis (FCDA), or combinations of the two to analyze the data. This chapter begins with a brief methodological explanation of CDS and its limitations. Next, I discuss the CL literature with an emphasis on its methodological considerations and the tools I use in this study. Next, I discuss how the limitations of both sets of approaches can be remedied through a synergy of them and review the benefits that each set of approaches provides to the other. Lastly, I describe my tweet collection process, turning these data into corpora, and how I analyzed it with corpus linguistic procedures and discursive constructions.

\subsection{Critical discourse studies}

Because critical discourse studies (CDS) are a group of approaches that seeks to "advance our understanding of how discourse figures in social processes, social structures and social change," it makes sense that there are many ways that scholars have critically studied discourse (Flowerdew \& Richardson, 2017, p. 1). These approaches are interdisciplinary because they bring multiple disciplines as well as their knowledge together to "[identify] issues of ideology, power, and inequality" (p. 1). Also, the fact that FCDA has no particular set of procedures associated with it illustrates the lack of a singular way to conduct CDS. In contrast to Discourse Analysis/Discourse Studies (DA/DS), CDS (and likewise, FCDA) is problem-driven, and rather than only examining linguistic units, these 
approaches "[aim] to uncover hidden features of language use and debunk their claims to authority" (Flowerdew \& Richardson, 2017, p. 1). For example, FCDA aims to demonstrate "the complex, subtle, and sometimes not so subtle, ways in which frequently taken-for-granted gendered assumptions and hegemonic power relations are discursively produced, sustained, negotiated, and challenged in different contexts and communities" (Lazar, 2007, p. 142). However, the discourses that practitioners investigate do not necessarily have to be negative or an "exceptionally 'serious' social or political experiences or events," as critical is not synonymous with negative (Wodak \& Meyer, 2015, p. 2).

Though CDS analysts investigate linguistic units, research may also include the concepts of lexical style, argumentation, and grammatical structures. Of course, analysts should not undertake such investigations without considering the context's influence on these categories. I summarize a few points by adopting Fairclough's view of discourse (Section 2.2.3) to explain the context of the protest and counter-protest of the present study and its social, political, and historical dimensions, I summarize a few points. Firstly, the analysis of the text examines tweets which individuals ambiently affiliate around the discourse topic of the sexual harassment allegations attributed to Mignogna. Additionally, this discourse topic is the content that generates the \#KickVic affinity space. Secondly, the analysis of discourse practices examines hashtags (production) that individuals use in the service of digital activism (distribution and consumption), especially those for social issues around gender and sexual harassment. Furthermore, this analysis examines Twitter's design and how it constrains, promotes, and shares discourses. Lastly, an analysis of social practices entails the larger conversation around 
the social issue of sexual harassment and how individuals are advocating for awareness and change within society.

Fairclough (1992, as cited in Flowerdew \& Richardson, 2017) stresses how important history is to analysis, demonstrating that at the first level of analysis (the text), "intertextual relations highlight changes in social structure" (p. 6). Context is crucial for any project that studies hashtags used for digital activism because individuals interpret the meanings of these hashtags according to social issues and the actions of people that reinforce those social issues. As well, because \#KickVic is only one instance of people speaking out concerning the lack of accountability for sexual harassers, it too is situated within the larger conversation of \#MeToo. While digital activism provides safety for marginalized groups who address the issues surrounding sexual harassment, previous and successful hashtags used in the service of digital activism provide meaning to the discussion and another pillar of reassurance.

Despite the wealth of knowledge that CDS approaches can provide to critical analyses of social issues and digital activism, it is also not without its criticisms (Baker et al., 2008; Koller \& Mautner, 2004; Partington \& Marchi, 2015). As I address in the following sections, one way to mitigate these limitations and biases is the inclusion—and synergy—of corpus linguistics (CL) alongside CDS approaches.

\subsection{Corpus linguistics}

Of the many branches of linguistics, corpus linguistics (CL) is the study of language where the primary source of data is a corpus. A corpus (plural corpora) is a collection of linguistic data that has internal consistency. Most importantly, according to Hunston (2002, as cited in McEnery et al., 2006), is that "a corpus is always designed for 
a particular purpose" (p. 59). As such, its usefulness must be assessed regarding "the purpose to which a user intends to put in" (p. 59). Here, I created purpose-built ("specialized") corpora with specific criteria that have internal consistency such that I could compare and contrast them.

Both considered a theoretical approach (Stubbs, 1993, pp. 23-24; Teubert, 2005, p. 2, as cited in Biber \& Reppen, 2015) and a methodological approach, CL aims to empirically investigate "language variation and use," with the goal that findings produced "have much greater generalizability and validity than would otherwise be feasible" (p. 1). In contrast to CDS/FCDA, CL uses quantitative methods to identify linguistic patterns that support "the view that language variation is systematic" (p. 2). Using collections of natural texts to inform language descriptions is not a new practice, and neither is corpus linguistics. Modern (corpus-based) CL is not a new development either because researchers have created and examined large electronic corpora since the 1960s. These modern corpora include Kučera and Francis’s (1967, as cited in Biber \& Reppen, 2015) Brown Corpus, a 1-million-word corpus of published American English (AmE) written texts, and the parallel Lancaster-Oslo/Bergen (LOB) corpus compiled of British English (BE) written texts published in the 1970s.

As detailed in McEnery et al. (2006), a corpus can take on many forms "which are classified in terms of their potential use" (p. 59). Therefore, the classifications of a general (or "reference") versus a specialized corpus are the most relevant to my study. Considering a language variety, using British English as an example, a general corpus is balanced, meaning that the genres in the corpus represent a range. For this, the corpus may contain written and spoken data and/or several categories of text. Comprised of 
$100,106,008$ words from 4,124 written texts and transcripts of speech, the British National Corpus (BNC) aims "to represent as wide a range of modern British English as possible" (p. 59). In contrast to this, "a specialized corpus is specialized relative to a general corpus" and contains genre- or domain-specific texts representing a variety of a language (p. 60). For example, the corpora I constructed for this study constitute specialized corpora as they contain tweets sampled from two hashtags, and every text (tweet) in these corpora has one or more of those hashtags.

Due to its empirical aims and quantitative methods, CL uses statistical tools to explore linguistic patterns and variations in a corpus. Just as the design of the corpus is informed by the research question, so too should the question(s) inform the researcher what CL procedures need to be conducted. As such, not every study that employs CL will use all the possible tools in its repertoire. On top of this, a researcher must remember that the CL procedures do not merely generate numbers; each procedure has implications. One of the central assumptions of corpus linguistics is that "formal distributional differences reflect functional differences [;]" that is to say, formal elements (morphemes, words, syntactic patterns, etc.,) have different frequencies because they reflect different functions (semantic, discourse-pragmatic, etc.,) (Gries, 2009, pp. 1225-1228). Assumptions like this make CL both a methodology and a set of methods because each procedure and tool has a set of assumptions behind them. For example, a frequency word list gives the frequency of every word in the corpus (Baker, 2006). When using a stop word list, the wordlist provides the frequencies for all the words not on this list. A stop word list is a list of words, usually those common in a language and/or of the functional class type, that the researcher does not want to count. A frequency list provides a general 
overview of the corpus's content and highlights the frequent words in that specific corpus. Considering content words - and not functional- I assume that high frequency does not mean a word is generally frequent in all language use. Instead, I believe high frequency content words imply that they are not backgrounded and assumed by speakers (and vice versa, low-frequency words are when speakers assume them a lot).

Another procedure of this project is the keyword list, where keyword means that the word is unique to a corpus when I compare that corpus against a reference corpus or a reference word list (Baker, 2006). Likewise, a reference corpus is a corpus that a CL tool uses as a reference to determine how 'unique' a word is in the target corpus (the corpus under investigation). Similarly, a reference corpus can be general (the texts that make it up are not of a single type, field, or registers) or specialized (the texts are of one or more types, fields, or registers). Like frequency counts, a keyword list informs the researcher of the unique keywords in a specific corpus in comparison to a reference point. One way the keyword procedure can calculate this uniqueness is "by comparing the word frequencies of two corpora and determining if the differences between frequencies are statistically significant" (Vessey, 2019, p. 320). Also, the reference point is important because generating keywords from a domain-specific reference corpus (e.g., textbooks) will provide keywords that are unique only in comparison to the words of that reference corpus. Lastly, generating keywords for two related corpora against the same reference point could show that the 'unique' keywords are 'unique' in both corpora, challenging whether they are truly unique. As I explain in the sections about corpus linguistics procedures, I use the keyword procedure to highlight similarities and differences between my three specialized corpora of tweets. 
To understand how words are used in a corpus, a researcher can conduct a collocation analysis. The procedure that produces collocates represents "a lexical relation between two or more words which have the tendency to co-occur with a few words of each other in running text" (Stubbs, 2001, p. 24). Collocates provide insights into what other lexical items co-occur around a target item. Additionally, this analysis can show how these lexical items are used around the target word, which can be determined by whether these items occur to the left or right of the target word. For example, if performing a collocate analysis on the noun women, verb collocates that appear to the left of the noun will likely be actions done to women while verb collocates that appear to the right of the noun will be actions that those women are the agents of. CL analysts measure collocations through "relationships of frequency and exclusivity" that in one way can be measured through the mutual information (MI) score (Baker, 2010, p. 24). With a score of 3 or more considered to be significant (Baker, 2006; McEnery et al., 2006), this value is "calculated by examining all the places where two potential collocates occur in a text or corpus" and then determining "the expected probability of these two words occurring near to each other would be, based on their relative frequencies and the overall size of the corpus" (Baker, 2006, p. 101). While a collocate of a word can have the highest MI score, it is also possible to sort the collocates by frequency to examine collocates that are the most frequent and most significant of a target word. Because the literature on statistical tests for CL develops continuously and rapidly, there has yet to be a consensus on the best tests to use for this procedure.

The last relevant CL tool to this project is the concordance line. Simply put, a concordance line is a line of the text from a corpus that a researcher uses to analyze a 
keyword in context (KWIC). All these lines together are called a concordance (Baker, 2006). CL software can expand the surrounding context of the search term to the left and the right if the researcher needs more context to interpret the word's meaning. Though concordances allow researchers to examine words in detail, I believe that for tweets it is sometimes necessary to consult the entire "text" (i.e. the entire 280-character span) because intertextuality is abundant on Twitter (Zappavigna, 2011). Entwined notions to corpus linguistics include semantic preference (Stubbs, 2001), semantic prosody (Louw, 1993), and discourse prosody (Stubbs, 2001). In linguistics, semantics studies the meaning and reference in language. Concerned with "the relation, not between individual words, but between a lemma or word-form and a set of semantically related words" is semantic preference (Stubbs, 2001, p. 65). Related to this, CL analysts can use collocations and concordances to examine "items that do not belong to a semantic set, but which have in common a particular attitudinal meaning" called semantic prosody (Baker, 2006, p. 251). To “emphasize [these prosodies'] function in creating discourse coherence," or logic and consistency, Stubbs prefers the term discourse prosody, "a feature which extends over more than one unit in a linear string" (Stubbs, 2001, pp. 6566). Similar to semantic prosodies, discourse prosodies also "express speaker attitude," is evaluative, and "express the speaker's reason for making the utterance" which in turn "[identifies] functional discourse units" (p. 65). Coupled with quantitative frequencies and collocations, qualitative analysis from concordance lines provides evidence concerning how individuals use words within a corpus. 


\subsection{The synergy of CDS and CL}

With an increase of CL techniques in critical approaches to discourse analysis (Baker et al., 2008), it becomes crucial to determine how to synergize CL and CDS for a research project. My study follows the view of Baker et al. (2008), where the CL techniques generate a map of the corpus that, in the process of performing $\mathrm{CL}$ procedures, pinpoints areas for close analysis using CDS/FCDA. In the context of my study, this takes the form of quantitative analysis of word frequencies, semantic themes, keywords, and collocates from the entire corpus to qualitative analysis of one keyword via collocates from the three corpora as well as discursive constructions and representations of social actors with a down-sampled selection of tweets. Of importance to Baker et al.'s synergy of CL and CDS is that they contribute equally and distinctly to the analysis and that one is not "subservient to the other" (p. 274).

One reason to explore the synergy between CL and CDS is that by using complementary approaches and methods associated with each, studies can mitigate the criticisms of CL and CDS. With regards to the criticism that CL tends to disregard context (Mautner, 2007; Widdowson, 2000, as cited in Baker et al., 2008), examining concordances or whole texts via CDS helps "to sufficiently recreate the context" (Brown \& Yule, 1982, as cited in Baker et al., 2008, p. 279). CDS is not without its criticisms either, ranging from the analyst's prejudice (Partington \& Marchi, 2015), to the selected texts for analysis, the representativeness of these texts (Koller \& Mautner, 2004; Stubbs, 1997, as cited in Baker et al., 2008, p. 281), and the number/size of texts selected (Stubbs, 1994, 1997, as cited in Baker et al., 2008, p. 283). CL procedures can reduce the chances of bias text selection and increase representativeness in the findings because the 
CL program (not the researcher) conducts the procedures and identifies the initial patterns in the data. With CL, CDS practitioners can also increase the number and size of texts they analyze, resulting in quantifiable findings of linguistic patterns in a set of texts. Overall, both CL and CDS benefit from a successful synergy where they both supplement attributes that the other lacks, which is why I used this approach for my study.

\subsection{Collection tools for tweets}

For this project, I collected tweets with automated tools. There are many tools on the web to collect tweets, and each has its benefits and difficulties. For example, the collector must consider how old the tweets that they want to save will be by the time they begin the collection process as this will affect the tools that they can use to achieve this. Additionally, some tools require knowledge of the programming language Python and how to use the command prompt to run Python scripts. Lastly, a Twitter scraper that does not rely on Application Programming Interface (API) to collect tweets might encounter more problems throughout the collection or even in the future as Twitter updates the code for their website. Scrapers that collect tweets as individuals create them are less likely to encounter this issue as the Search API (what people use to access tweets) is available for a predetermined number of days. Hawksey (2014) says that the index period that a Search API generates includes tweets that are six to nine days old while Taspinar (2020) remarks that this period is seven days. This factor does not make Twitter scrapers that do not rely on Search API less reliable. Instead, it means that the researcher needs to have a basic understanding of coding. Regardless, a corpus creator must decide to either pre-emptively collect data or hope that it is possible to collect older tweets without too many difficulties, and all of this within a rapidly changing social media environment. 
Because I collected tweets from two different points in time-when \#KickVic and \#IStandWithVic began in January 2019 and at the conception of this project in January 2020 - I used two collection tools to gather the tweets. At the time of collection (January 2020), the tweets from 2019 were over ten days old and it was far too late to use a scraper that collocated tweets as users posted them. As such, the two tools I used to collect the data for this research were Twitter Archiving Google Sheet (TAGS) (Hawksey, 2014) and TwitterScraper (Taspinar, 2020). I used TwitterScraper to collect the tweets from 2019 and used TAGS to collect tweets from 2020.

\subsection{Sampling}

When conducting a corpus linguistics study, it is difficult to judge when there is enough data to use for research because the project and its circumstances will vary in several factors. A review of the CL literature shows no consensus concerning the amount of data necessary that a researcher requires to perform an adequate analysis and Hunston (2008) suggests good reasons for this. For example, a group of researchers can sample more data for their project than an individual conducting the same analysis because the workload is manageable; however, this is not to say a one-person research team needs to limit themself to a small data sample. There are also limits for these projects. For example, it is unlikely that a study will be able to sample every instance of a particular word, phrase, or discourse that has ever occurred, and so it is important to be satisfied with the amount one can sample. Also, not collecting every example leaves room for the researcher or other researchers to conduct new studies in the future.

Considering the above, I sampled as much data as possible before I began data clean-up. To collect as many tweets as possible from 2020 (individuals created far fewer 
tweets with the hashtags during this year than in 2019, as I show below), I allowed TAGS to collect tweets for six months, from January $28^{\text {th }}, 2020-J u l y ~ 28^{\text {th }}, 2020$. I created two sheets: one to collect tweets with the search term \#KickVic and the other with the term \#IStandWithVic. I told TAGS to update the archive for both spreadsheets every hour and this resulted in 28169 tweets before I cleaned them. \#KickVic had 5,991 tweets over six months, and \#IStandWithVic had 22,179 tweets. Of note, these totals include all retweets, which is an element I later cleaned from my data; I detail my reasoning for this in the Cleaning and sorting tweets section. During this period, I also collected the tweets from 2019 and did so in two separate batches: one for each search term. After successfully using TwitterScraper, I had collected 24,652 tweets: \#KickVic had 11,937 tweets and \#IStandWithVic had 12,717 tweets. Unlike TAGS, TwitterScraper does not extract instances of retweets; as such, the total for the 2019 tweets are all unique instances. However, tweets in the original collection might contain both hashtags (\#KickVic and \#IStandWithVic), and therefore there are some repeated tweets across the two datasets. In Section 3.7, I explain how I cleaned and sorted these tweets into three corpora without repeating individual tweets.

\subsection{Ethical considerations for Twitter data}

Considering discussions of privacy and ethics for digital work on social media, my corpora do not contain the tweeter's identity or the tweet's timestamp; the only data I kept for the corpora is the text of the tweet. As an individual, I realize that while I have legal permission from Twitter to collect and analyze data because it is public information, this does not mean it is ethical, as users are unaware of my research and may feel uncomfortable that I am collecting their data without their consent (Ruest \& Milligan, 
2016). As such, I take every precaution to protect user's identities, especially for those individuals who could face harm in situations of unequal power. These precautions include, for example, if a user appears in the word frequency list, keywords for the corpus, or as a part of examples, then I substitute it for a pseudonym using the format individual_A, individual_B, etc. As such, every person (aside from Mignogna) has a letter assigned to their name. Also, letters are not correlated to names; instead, the first individual I designated letter $A$, the second I designated as individual_B, etc. While this partially mitigates the risk of identification, it is also possible to find a tweet if searching for a particular string of words on Twitter, so long as the individual has not made their account private (only individuals the user has approved of can interact with their tweets) or deleted their tweet. Although many tweets exist with a certain string of words, it can be obvious which one(s) are a part of my corpora because, as the purpose of this project designates, all tweets must contain either \#KickVic or \#IStandWithVic. Therefore, if the need arises to show examples, I aim not to share a particularly obvious string of words and cross-check on the platform to ensure that the tweet cannot be found.

The reason I am adamant about protecting user identities as they relate to my data —which also influences the metadata I removed from the tweets - is because of the tweeters who are a part of this affinity space. Crucially, I remind my readers that the \#KickVic hashtag began as a space where tweeters discussed the sexual harassment allegations attributed to Mignogna to create awareness concerning the issue and to demand accountability. Given that two women who were Mignogna's coworkers posted to Twitter allegations involving him and that these two women were a part of the group whom Mignogna sued, it is not difficult to imagine that other women have had similar 
experiences and thought the \#KickVic hashtag an appropriate space to share them. As well, this affinity space provides safety to these women who are marginalized and oppressed because if they were to report the same experiences to authority figures faceto-face, like police, there is the potential that "they won't be believed and that the voices of men with status will be believed over theirs" (Canadian Women's Foundation, 2021). Believing that it is necessary to respect tweeters' decision to "[possibly] remain anonymous on Twitter" during these discussions (Palomino-Manjón, 2020) as well as wanting to minimize opportunities for cyberbullying as my thesis will be available online, I decided to protect tweeters' identities, to the best of my ability, during the construction of my corpora. For these reasons, I removed usernames and timestamps from the tweets, modified examples used in Chapters 4 and 5 after I completed the analysis, and do not show word-for-word concordance lines anywhere within this document.

With these ethical considerations in mind, it is also necessary that I address the consequences these decisions have on analysis. Firstly, because I removed usernames from my corpora, it is impossible to control or detect potential imbalances in the generated frequencies. Because of this ethical decision, I cannot know if a result, for example, a high word frequency, is because tweeters generally use it a lot in the tweets I collected or if the high frequency is due to one tweeter or a group of tweeters continuously posting it. Therefore, I must remember that any results I find could be skewed because of this factor. Future research or a repeat of this study can alleviate this potential of high-volume contributors by surveying the tweets before removing username metadata to determine the extent to which users are a part of the corpus. Secondly, I 
removed timestamp metadata from the tweets to further protect tweeter identity and anonymity so that, hypothetically speaking, a person with ill intent has a more difficult time searching for a tweet because specific timeframe information is not available. However, this ethical decision has consequences in that I could not perform any time course-related analysis; I mention an example of this in Section 3.9 where I had to use a different method to down-sample tweets. As I do not include time as a factor in my research questions, I do not believe this timestamp issue is analytically relevant to my study. With this in mind, performing an analysis on these tweets that considers their timestamps is possible so long as the author does not include timestamps with tweets should the author use text examples.

Lastly, as I detail in Section 3.8, I removed all instances of recorded retweets that TAGS collected. Though I made this decision more from a consistency perspective (TwitterScraper does not record retweets) than an ethical one, I believe it has ethical implications and analytical consequences. Regarding ethics, it is possible to search for tweets via engagement filters using Twitter's advanced search options and one of these options includes specifying a 'minimum retweets' number. Should I identify that certain examples have a particular amount of retweets, it gives people more information that makes it easier to find a specific tweet. However, the extraction of retweets from my data means that there is a loss of network structure in that the centrality of certain tweets to the protest and counter-protest, as well as their influence, becomes impossible to analyze. Again, because the research questions that focus my study do not involve centrality and influence, I was not worried about this loss from an analytical perspective. However, analyzing retweets is a potential research project I could conduct following this study, 
and I think its findings would have a lot to contribute to the current research because of the importance the theoretical concept of affinity spaces has to my work. Overall, I believe that in the future, many of the consequences my ethical considerations created can be remedied so long as data management and the presentation of the data is handled mindfully and with great care to protect the tweeters' identities and anonymity.

\subsection{Cleaning and sorting tweets: A necessary struggle}

Before I sorted my data into the three corpora, I cleaned it to make it easier to analyze for both CL tools and discursive constructions. Cleaning data can take many processes and the procedure I followed used Microsoft Excel with the help of Microsoft Word to delete page breaks and line breaks. The list of procedures I completed in Microsoft Excel includes deleting all tweets that were retweets, deleting duplicate tweets, converting character encodings back into UTF-8, and sorting tweets into corpora based on the hashtags they contained. I provide a detailed explanation of these procedures in Appendix B. This explanation does not include difficulties I encountered with spelling variation as, admittedly, I spend a lot of time on Twitter and I usually understand what these variations mean. After I finished these procedures, I saved the data in a text file with UTF-8 encoding using Notepad. It is these text files that are my corpora and that I analyzed.

To combine the four collections of data (two sets from 2019 and two sets from 2020) into corpora, I separated tweets based on the hashtags they contained. To this end, I created three corpora. The first, called the \#KickVic corpus, contains tweets with \#KickVic and possibly other hashtags but not \#IStandWithVic. The \#KickVic corpus contains 7,720 tweets and 244,990 word tokens and represents the discourse of the 
protest. The second corpus, called the \#IStandWithVic corpus, contains tweets with \#IStandWithVic and possibly other hashtags but not \#KickVic. This corpus is the largest, with 14,497 tweets and 383,739 word tokens and represents the discourse of the counterprotest. The last corpus, called the mixed corpus, contains tweets that have both the \#KickVic and \#IStandWithVic hashtags as well as possibly others. The mixed corpus is the smallest, with 5,310 tweets and 171,514 word tokens; also, its discourse cannot be easily described as it contains elements from both the protest and the counter-protest. Admittedly, these corpora are not perfect, as during their construction I did not account for alternative versions of the hashtags like \#KV (for \#KickVic) and \#ISWV (for \#IStandWithVic). I address the limitations of this decision in Section 6.2. Despite the size differences of the corpora, normalized word frequencies make it possible to compare them. Also, the size differences are considered a finding and I discuss this in Chapter 4.

Returning to the construction of the corpora, the reason for the separation of hashtags is that I am interested in the protest (\#KickVic) and counter-protest (\#IStandWithVic) discourses of this event as well as the discourses that straddle a possible boundary between both. As such, I extracted tweets that met the requirements of each corpus using the Sort \& Filter function in Excel. This process worked for the most part, but I sorted a handful of tweets into the correct corpus manually because of Excel's limitations. I encountered this problem when constructing the \#IStandWithVic corpus as when I filtered out those tweets that contained the hashtag \#KickVic the software also filtered out tweets that used other hashtags that contain \#KickVic, like \#KickVicMoron, which is not the same hashtag. I believe Excel did this because \#KickVic is contained within a hashtag like \#KicVicMoron and therefore does not distinguish the two as 
different items. As Excel is better at working with numbers than words, this issue is not a surprise and though it did slow down the process of corpora construction, I identified several derivatives of the hashtag \#KickVic through this process which I will discuss in later chapters.

The reason why I fixed many of these issues without completely restarting the cleaning/sorting process is for two reasons. Firstly, every time I completed a different procedure, I saved that file as a new copy rather than saving it on top of the original file. For this reason, if I noticed a mistake, I returned to the previous version of the file and did not begin the cleaning process again from the original file. Secondly, as I collected, cleaned, and sorted my data, I kept a separate metadata file where I recorded every choice I made and why I made it. This file proved beneficial when I needed to repeat a process in a new spreadsheet and served as a point of reference to the decisions I made when writing the current chapter. A metadata file provides a space for researchers to write notes along the way about their project and serves as an excellent source of reference so that they do not have to depend on their memory, especially when constructing a longterm project like this one. As such, I encourage the creation of metadata files for all research projects and not only those working with social media data.

\subsection{Corpus linguistics procedures}

Following the logic from Baker et al. (2008) that the CL analysis creates a roadmap that identifies areas for in-depth CDS (in my case, FCDA) investigation, I used an approach informed by Vessey (2019) for this first part of the analysis. Vessey's methods involve many CL tools (word frequency lists, keywords, collocates, concordance lines) and she structures her analysis strategically, allowing for triangulation 
of the data. Using Vessey (2019) to inform my methods proved most beneficial because she also compares the similarities and differences between two related corpora. Below, I outline the steps I followed that, despite modifying Vessey's original procedure, still provided informed insights to potential areas of closer investigation amongst the entirety of the dataset, quantifiable numbers of patterns, and a lower chance for bias in the selection of tweets for in-depth FCDA examination.

Because Vessey's (2019) study served as a guide for my own—as well as time constraints and ease of access-I decided to also use the AmE06/BE06 wordlists as a reference corpus in my study. Initially, this caused some concern since these corpora underrepresent contemporary online genres and texts like tweets where the length is because of platform constraints. However, I believe using the AmE06/BE06 wordlists alongside more Twitter-specific corpora (for more information, see the following paragraph) to generate keyword lists reveals what keywords related to Twitter conventions are unique to my corpora and what keywords within the context of this affinity space are unique to my corpora. For example, an AmE06/BE06 comparison might show username or hashtag keywords whereas comparison with a Twitter corpus can focus on those keywords that are more specific to this protest and counter-protest. Using my corpora as reference corpora also partially mitigates the need to rely on another researcher's Twitter corpus where I cannot be sure if our ethical considerations align for social media constructed corpora. My reasoning for why I believe these corpora are acceptable reference corpora options is also informed by Scott (2009). In this literature, Scott (2009) discusses that rather than approaching the situation from the view that a reference corpus is a bad choice, we should view it from the perspective that there are 
different comparisons to be made and findings to be discovered. With this information in mind, I think the decisions I made were adequate given the time and resources available and respecting my ethical considerations.

Vessey's procedure can be categorized into three main steps. First, investigate the first corpus (for my analysis, \#KickVic) in its entirety, including word frequency, keyword, collocate, and concordance line analysis. Next, investigate the second corpus (\#IStandWithVic) in its entirety. Lastly, directly compare the two corpora against one another. Altogether, this procedure includes nine steps. For the CL portion of my analysis, I used all these steps with the addition of also investigating the mixed corpus in its entirety, resulting in extra stages of analysis. Also, I used each corpus as a specialized reference corpus at certain stages of the analysis to examine how the corpora are similar and different from each other. An enumeration of the CL procedures I followed is below. The order of these steps is how I conducted the analysis; the report of the findings from these procedures does not follow this order in Chapter 4.

1. Perform analysis of each corpus individually

a. Generate a word frequency list for the \#KickVic corpus

b. Using the AmE06/BE06, generate a keyword list for the \#KickVic corpus

c. Analyze the top 200 keywords via collocates and concordance lines; sort these keywords into semantic themes

d. Generate a word frequency list for the \#IStandWithVic corpus

e. Using the AmE06/BE06, generate a keyword list for the \#IStandWithVic corpus 
f. Analyze the top 200 keywords via collocates and concordance lines; sort these keywords into semantic themes

g. Generate a word frequency list for the mixed corpus

h. Using the AmE06/BE06, generate a keyword list for the mixed corpus

i. Analyze the top 200 keywords via collocates and concordance lines; sort these keywords into semantic themes

j. Compare the semantic themes and top 200 keywords from each corpus together to assess similarities and differences

k. Compare the semantic themes and keywords across the corpora in pairs of two to assess similarities and differences

i. Categorize the unique keywords to the \#KickVic corpus

ii. Categorize the unique keywords to the \#IStandWithVic corpus

iii. Categorize the unique keywords to the mixed corpus

2. Perform analysis via direct corpus comparison

a. Generate keyword lists for the \#KickVic corpus

i. Use the \#IStandWithVic corpus as the reference corpus

ii. Use the mixed corpus as the reference corpus

b. Analyze the keywords via collocates and concordance lines; sort these keywords into semantic themes

c. Generate keyword lists for the \#IStandWithVic corpus

i. Use the \#KickVic corpus as the reference corpus

ii. Use the mixed corpus as the reference corpus 
d. Analyze the keywords via collocates and concordance lines; sort these keywords into semantic themes

e. Generate keyword lists for the mixed corpus

i. Use the \#KickVic corpus as the reference corpus

ii. Use the \#IStandWithVic corpus as the reference corpus

f. Analyze the keywords via collocates and concordance lines; sort these keywords into semantic themes

g. Compare the semantic themes and keywords from each corpus together to assess similarities and differences.

h. Compare the semantic themes and keywords across the corpora in pairs of two to assess similarities and differences

i. Categorize the unique keywords to the \#KickVic corpus

ii. Categorize the unique keywords to the \#IStandWithVic corpus

iii. Categorize the unique keywords to the mixed corpus

In comparison to Vessey's procedure, my analysis involved more comparisons of keywords and semantic themes. To determine the similarities and differences of the three corpora taken together, I also needed to compare the corpora in pairs. This 'pairs of two' comparison provided the chance to examine similarities between, for example, only the \#IStandWithVic corpus and mixed corpus and not the \#KickVic corpus. This procedure also allowed for the investigation of differences to discover words that were truly unique to a corpus.

Using AntConc version 3.5.9 as the toolkit to carry out CL procedures (Anthony, 2020), I adjusted its settings so that it would be better equipped to work with social media 
data. These changes come from the suggestion of Silva (2019) who created a file with updated settings that I imported directly into AntConc. These settings alter the token definition in global settings to include symbols that commonly appear in social media texts like the '@' symbol and the '\#' symbol. Without these changes, it would not be possible to count or filter out the number of mentioned Twitter users or hashtags as the program with its default settings does not consider these symbols to be important. Also, the '@' symbol and '\#’ are, in the default version of AntConc, attributed to wildcard settings. In corpus linguistics, wildcards are characters that serve as placeholders for other characters. For example, when searching the string $\mathrm{cat}^{*}$, AntConc displays all instances of cat and cats in the corpus. It is therefore important to change the wildcard settings for these two symbols to others, like curly brackets, so AntConc does not ignore these two signs.

The first step of each corpora's analysis for my study, as with almost any corpus linguistics analysis, is to generate a word frequency list, referred to as a word list in AntConc. Because this procedure analyzes one corpus completely at a time, I carried out this first step with the \#KickVic corpus. I also conducted this procedure with the \#IStandWithVic corpus and the mixed corpus. Importantly, in AntConc, it is necessary to generate a word list before performing the keyword procedure because the program needs to know the frequencies of the words in the corpus. As such, this first step is necessary to carry out the next one.

\subsubsection{Keyword analysis}

The second step of each corpora's analysis involves generating a keyword list against a reference corpus. Because of how I separated my data, the opportunity 
presented itself to compare each corpus against general and specialized corpora. The general reference corpus I use is the combined wordlists of the AmE06/BE06 (both are available to download from Paul Baker's homepage). Developed by Baker (2009), the BE06 word frequency list comes from the BE06 Corpus, "a one million word reference corpus of general written British English that was designed to be comparable to the Brown family of corpora" (p. 312). Similarly, the AmE06 word frequency list comes from the AmE06 Corpus, developed by Potts and Baker (2012) "to be [an American] companion corpus to the [BE06 Corpus]" (p. 301). The specialized corpora I used as reference corpora are the corpora I constructed. As such, there are three keyword lists for each corpus; using \#KickVic as an example, there is one keyword list from when compared against the combined wordlists of the AmE06/BE06, one from when compared against the \#IStandWithVic corpus, and one from when compared against the mixed corpus. The \#IStandWithVic corpus and the mixed corpus also each have three keyword lists but against different specialized corpora. The purpose of this step is twofold: first, the keyword lists generated from the comparison against the three reference corpora establishes "the extent to which a corpus contains unique lexical content" (Vessey, 2019, p. 320). Second, using \#KickVic again as the example, the keyword lists generated from the comparison against the \#IStandWithVic corpus and mixed corpus have the potential to establish whether this unique lexical content is unique to the target corpus or perhaps this protest and counter-protest more generally. I generated the keywords for each comparison with AntConc's default settings for keyness values: log-likelihood (4-term), $\mathrm{p}<0.05$ (+Bonferroni), dice coefficient, all values. For the remainder of this explanation, I speak solely about the keyword lists generated from the comparison against AmE06/BE06 
wordlists. I return to the list of keywords generated from the comparison against the specialized corpora later.

\subsubsection{Semantic themes}

With a list of keywords generated, I began to categorize these keywords into semantic themes. The semantic themes from the similarities comparison include references to Mignogna, evidence, groups of people, protest, (affinity) spaces, attributes, harassment, pieces of web addresses, a collective, specified individuals, and actions. I generated a similar list of semantic themes for the keywords that were truly unique to each corpus, though there are a few differences. These semantic themes include (affinity) spaces, harassment, actions and attributes, people, discourse, the protest, and other. For certain keywords, like those referring to Mignogna, it was easy to categorize them into semantic themes. However, I found it necessary to analyze collocates and concordance lines for some keywords when their meaning was not obvious. For example, I needed to know whether the keyword pic (short for picture) was a part of a web address or if tweeters used this lexical item to refer to a picture. In this analysis, I set the minimum collocate frequency to five hits, meaning that if a word co-occurred with my target word fewer than five times, it would not appear in the results. However, before making conclusions from the collocates of a target word, I also investigated how the target word and the collocates are used together in context. For these less obvious keywords, I examined their top ten statistically significant collocates, their concordance lines, and sometimes the entire tweet when I required more contextual information to understand the keywords' usages. Starting with the keywords generated for the \#KickVic corpus, I completed this procedure with each of the three corpora individually. 


\subsubsection{Similarities and differences}

Having categorized the top 200 keywords from each corpus into semantic themes, I compared the similarity findings within all three corpora when each used the AmE06/BE06 as a reference corpus (i.e. when I compared each corpus against the same reference corpus). Because the themes across the three corpora were very similar, I examined the keywords to determine those that existed in all three corpora. These similarities show how the three corpora are like one another in comparison to general discourse. At this stage of analysis, I could answer the question: What semantic themes are present in each of the three corpora? To understand how this question relates to my three research questions (Section 1.2), see Appendix A.

With this portion of the analysis complete, I compared the semantic themes and keywords across the corpora in pairs by examining their top 200 keyword lists. For similarities, I compared two keyword lists at a time to discover what the two corpora shared. I completed this procedure in the following order: the \#KickVic corpus and the \#IStandWithVic corpus, the \#KickVic corpus and the mixed corpus, and the \#IStandWithVic corpus and the mixed corpus. The limitations of this approach are discussed in Section 6.2. Differences, or keywords unique to each corpus, were determined by similar means. Firstly, I compared the top 200 keywords of the \#KickVic corpus against the \#IStandWithVic corpus' keyword list and the mixed corpus' keyword list and set aside the keywords that were only keywords from the \#KickVic corpus. After this, I determined if these unique keywords belonged to the categories of semantic themes that I had previously identified or if they were a part of a new category. Next, I followed the same procedure with the top 200 keyword list from the \#IStandWithVic corpus by 
creating two lists: the first contained unique keywords of \#IStandWithVic that were not keywords in the \#KickVic corpus and the second contained unique keywords of \#IStandWithVic that were not keywords in the mixed corpus. Again, I categorized these unique keywords into semantic themes. Lastly, I performed this procedure for the keywords of the mixed corpus where I created two lists of keywords. The first list contained unique keywords of the mixed corpus that were not keywords in the \#KickVic corpus and the second list contained unique keywords of the mixed corpus that were not keywords in the \#IStandWithVic corpus. The strength of this stage is that it identifies both similarities and differences between the three corpora (Vessey, 2019). Taking these three groups of semantic themes, I compared them to assess what distinguishes the three corpora, while also answering the questions: What semantic themes are present in the \#IStandWithVic corpus and what semantic themes are present in the mixed corpus? I address these sub-questions in the research design; see Appendix A.

\subsubsection{Direct corpus comparison}

For this stage of analysis, the CL procedures I performed involved a direct comparison of the corpora against each other. Taking the keyword lists I had previously generated by comparing each corpus against the other two, I rigorously compared all the generated lists of keywords (this resulted in six lists in total) against each other to determine if they shared any keywords. I examined all the keywords rather than only the top 200 because no comparison resulted in more than 161 keywords. To determine further similarities between the three corpora, I compared keyword lists in pairs of two. I conducted this analysis in the following order: compare the keyword lists from the \#KickVic corpus and the \#IStandWithVic corpus when they were each individually 
compared against the mixed corpus, compare the keyword lists from the \#KickVic corpus and the mixed corpus when they were each individually compared against the \#IStandWithVic corpus, and compare the keyword lists from the \#IStandWithVic corpus and the mixed corpus when they were each individually compared against the \#KickVic corpus. Like the previous similarity analysis, this investigation determined what a corpus had in common with another but was not shared by all three.

The last CL procedure of this direct corpus comparison established the unique keywords of each corpus when I compared the corpus against the other two. Once again using the six generated lists of keywords, I thoroughly compared these lists to determine keywords belonging only to the \#KickVic corpus, those that were only keywords in the \#IStandWithVic corpus, and the unique keywords of the mixed corpus. This procedure aided in determining how unique each corpus is in comparison to all the data collected concerning this protest and counter-protest.

By performing an individual analysis on each corpus and comparing the results, I could determine not only how they are similar to each other but also how they differ, compared to the AmE06/BE06 and discourse of the protest and counter-protest. Reporting the similarities of the three corpora is an important stage of CADS research, as argued by Taylor (2013). For example, although a keyword might be unique to a corpus when compared against a reference corpus, this same keyword could be as unique in the other target corpus under investigation, ultimately showing that the keyword is unique in comparison to the 'norm' (or the norm contained within a reference corpus) and regular for this specific protest and counter-protest. This finding could lead to the conclusion of specific keywords that individuals regularly use in digital activism hashtags, identifying 
available patterns to later examine in future research. For example, words related to digital activism and harassment could vary in uniqueness between the protest and counter-protest, highlighting how Twitter users not only discursively construct this event but also subvert or perpetuate ideologies that are the reason for this digital activism.

\subsection{Down-sampling}

Recalling the research questions, one aim of this study is to understand how tweeters discursively construct women in this protest and counter-protest. Because of the power asymmetries women face due to gender ideologies implicitly enacted through discourse in society, I find it highly relevant to analyze how people discursively construct them. I believe this is strongly the case for digital activism that works to create awareness for the social issues women encounter, especially in this instance where the digital activism hashtag for the protest received a response in the form of a counter-protest hashtag. Considering these factors, I focused on the discursive constructions (Section 3.10) of the term women in each corpus. Due to feasibility and time constraints, it was necessary to sample tweets containing women for stages of analysis involving CDS and FCDA. Across the three corpora, the plural form of this word is used 311 times and for one person this becomes a lot of work to analyze all of these individually. As such, I decided to down-sample how many tweets I analyzed, a method in corpus linguistics that a researcher uses "to reduce the number of articles to a workable amount" (Baker \& Levon, 2015, p. 225). This same study notes that “[while] down-sampling is certainly a form of "cherry-picking,," down-sampling can be carried out from an informed and objective position that decreases the extent of cherry-picking (p. 222). For example, authors have carried out down-sampling by selecting the high- and low-ends of the 
spectrum regarding how often a reference is made to a theme (Vessey, 2017), by allowing another program to decide what articles in a corpus "are the most representative of the entire set" (Baker \& McGlashan, 2020, p. 221), by certain periods or peaks (Baker \& Levon, 2015; Gabrielatos et al., 2012), and by clusters informed by collocation analysis (Forchtner \& Kølvraa, 2012). Because of how I collected and stored my data, none of these procedures were suitable for my project. For this reason, I determined what $10 \%$ of all the instances of the word women is for each corpus and selected that many tweets to analyze. However, $10 \%$ of all the instances of women in a corpus is not equivalent to the number of tweets that include women because sometimes a tweet includes the word more than once. Additionally, I rounded the generated percentage total up or down so that, for example, I did not have to analyze 0.2 of a tweet. Following this down-sampling procedure, I arrived at the totals represented in Table 2.

Table 2: Down-sampling totals across corpora

\begin{tabular}{cccc}
\hline & \#KickVic & \#IStandWithVic & Mixed \\
\hline Frequency of women & 152 & 120 & 39 \\
10\% of frequency & 15.2 & 12 & 3.9 \\
Tweets to analyze & 15 & 12 & 4 \\
\hline
\end{tabular}

After determining these values, I randomly sorted the tweets in each corpus and selected the required number of tweets that Excel randomly sorted to the top of the list.

\subsection{Discursive strategies}

After I completed the keyword comparison, patterns across the corpora started to emerge, and I pinpointed an area of focus for the FCDA analysis. During my analysis of collocates, concordance lines, and texts (tweets), I observed patterns and employed discourse analysis to identify the discursive strategies in use, with a particular focus on 
the social actor representation/discursive construction of women. Although FCDA has no particular set of discursive strategies of its own, I find the discursive strategies in the Discourse-Historical Approach (DHA) to be the most clearly defined in terms of their purpose and the line of questioning that approaches the features of discourse. Table 3 shows the discursive strategies as Reisigl (2017) outlines in his discussion of discourse according to the DHA. Briefly, this includes how items are referred to in discourse (nomination), the characteristics these items are attributed to in discourse (predication), the arguments used in discourse (argumentation), the perspective these previous strategies are expressed from (perspectivization), and whether the utterances are made overtly/covertly and intensified/mitigated (mitigation and intensification). For this project, I focus on the discursive strategies of nomination and predication to understand how women are discursively constructed and how they are characterized in this protest and counter-protest. I completed this analysis in a random down-sample of thirty-one tweets from the \#KickVic corpus, the \#IStandWithVic corpus, and the mixed corpus. Part of the nomination and predication strategies involve questioning how people linguistically refer to categories (people, objects, events, etc.) and the qualities these categories have. To survey what lexical items co-occur women and woman, I first conducted a collocate analysis and examined the top ten collocates of these target words across the three corpora. In this study, I only investigate those collocates that are content words rather than content and grammatical words, or only grammatical words.

Because of the focus on the discursive construction of one category of social actors (women), I also consulted van Leeuwen (2008) for his in-depth categorization of the representation of social actors. His work is grounded in linguistics where every 
representation is linked "to specific linguistics or rhetorical realizations" (p. 25). This framework includes the inclusion and exclusion of social actors from a text, which, depending on the purposes of the text, will have different implications for the audience. On top of being included, social actors can actively participate in the representation (activation) or "undergo" the activity (passivation). Of particular interest for this study is whether social actors are represented as a group (assimilation) or as individuals (specification) and the attributes that are linked to these representations. For example, some tweets refer to women as an aggregated or collectivized group while other Twitter users name specific individuals who are women in their tweets.

Table 3: Discursive strategies in the DHA (Reisigl, 2017, p. 52)

\begin{tabular}{|c|c|c|}
\hline $\begin{array}{l}\text { Questions to approach } \\
\text { discursive features }\end{array}$ & Discursive strategies & Purpose \\
\hline $\begin{array}{l}\text { How are persons, objects, } \\
\text { phenomena, events, } \\
\text { processes and actions named } \\
\text { and referred to linguistically } \\
\text { in the discourse in question? }\end{array}$ & nomination & $\begin{array}{l}\text {-discursive construction of } \\
\text { social actors } \\
\text {-discursive construction of } \\
\text { objects, phenomena, events } \\
\text {-discursive construction of } \\
\text { processes and actions }\end{array}$ \\
\hline $\begin{array}{l}\text { What characteristics or } \\
\text { qualities are attributed to } \\
\text { social actors, objects, } \\
\text { phenomena, events, } \\
\text { processes and actions } \\
\text { mentioned in the discourse? }\end{array}$ & predication & $\begin{array}{l}\text {-discursive characterization } \\
\text { of social actors, objects, } \\
\text { phenomena, events } \\
\text { processes [,] and actions } \\
\text { (e.g., positively or } \\
\text { negatively) }\end{array}$ \\
\hline $\begin{array}{l}\text { What arguments are } \\
\text { employed in discourse? }\end{array}$ & argumentation & $\begin{array}{l}\text {-persuading addressees of } \\
\text { the } \\
\text { validity of specific claims of } \\
\text { truth and normative } \\
\text { rightness }\end{array}$ \\
\hline $\begin{array}{l}\text { From what perspective are } \\
\text { these nominations, } \\
\text { attributions, arguments } \\
\text { expressed? }\end{array}$ & perspectivization & $\begin{array}{l}\text {-positioning the speaker's or } \\
\text { writer's point of view and } \\
\text { expressing involvement or } \\
\text { distance }\end{array}$ \\
\hline $\begin{array}{l}\text { Are the respective utterances } \\
\text { articulated overtly, are they } \\
\text { intensified or mitigated? }\end{array}$ & mitigation and intensification & $\begin{array}{l}\text {-modifying the illocutionary } \\
\text { force of utterances in respect } \\
\text { to their epistemic or deontic } \\
\text { status }\end{array}$ \\
\hline
\end{tabular}


Within the analysis of role allocation (activation and passivation) for women, I also found it beneficial to categorize the verbs they are the dynamic forces behind (agents) and the forces they undergo (recipients and beneficiaries) according to the terms used in Systemic Functional Linguistics (SFL). Although this analysis does not rely on this functionalsemantic approach to language, I found it beneficial to group the verbs using the system of transitivity established as part of experiential meaning because verbs are represented as a process type (Eggins, 2004, p. 213). In a clause, representations are not only made up of process types, but also the "functional participant roles" of a process "and the possible selection of circumstances" associated with it (p. 214). For this analysis, there are four processes I am concerned with: material, mental, verbal, and attributive. Material processes usually describe "concrete, tangible actions" where some entity "does something [or] undertakes some action" (p. 215). In the active voice, material processes typically require an actor and goal, though there are other possibilities for different participant roles. Contrastively, mental processes "encode meanings of thinking or feeling" and can represent verbs of cognition, affection, and perception (p. 225). Unlike material processes, a representation of a mental process includes a senser and a phenomenon. Thirdly, verbal processes are "processes of verbal action" and "typically contains three participants: [sayer], [receiver] and [verbiage]" (p. 235). Lastly, there are the attributive processes, which are a sub-type of relational processes because they represent "ways in which being can be expressed in English clauses" (p. 239). For the attributive sub-type, this process represents " $x$ [as] a member of the class a" or " $x$ carries the attribute $a$ " (p. 240). The categorization of verbs in this way allows for a critical 
analysis of the participant roles tweeters use to discursive construct women in this protest and counter-protest.

Although my focus is to analyze how Twitter users discursively construct women, one aspect of the discursive construction of social actors is whether users excluded them. Van Leeuwen (2008) distinguishes between two kinds of exclusion: suppression and backgrounding. The difference between these is whether the social actor is referenced in the text (suppression) or if they are present elsewhere in the text but not mentioned with an action that we would expect. While analyzing for the exclusion of a social actor is important, it becomes a question of whether tweeters did not include them because of exclusion or because women are not there since they do not need to be. In a digital protest on Twitter that uses hashtags to affiliate around the topic of sexual harassment, do we expect women to always be included? Given the lens of FCDA, I would say that we should expect women to be a part of the protest and counter-protest, though the expected extent to which they are included I am unsure. However, I will focus on how Twitter users discursively construct women to the extent that they are included while noting that this extent could have other implications. This includes the notion and existence of "absence" that CL tools locate and verify across different corpora as Duguid and Partington (2018) discuss.

This down-sampled analysis also examines discursive constructions of van Dijk's (1998) “ideological square” (p. 267). I identified instances of positive Us representation in consideration of the lexical content belonging to certain social actors. During this, I also examined how these social actors constructed the social actor of women (Them) through the actions or behaviours that they were associated with. If the tweeter criticized 
the actions and behaviours of women while representing another collective of social actors as against and harmed by them, I noted this down as an example of Us versus Them. Similar to Gallagher et al.'s (2018) study, I want to know if and how Twitter users discursively construct this Us versus Them mentality in these hypothesized divergent hashtags.

\subsection{Summary}

In this chapter, I explained the purpose of corpus linguistics as it relates to this project as well as the required synergy between CL and CDS/FCDA that is needed when using these two sets of approaches together. Next, I discussed how I collected my data and created my three corpora from it. During this, I also highlighted considerations I believe any researcher working with social media data need to be aware of before, during, and after this process. I also detailed how I performed CL and FCDA analysis on my data. This started with using the findings from the CL procedures to create a roadmap of the data and then selecting one area of interest to focus on. To apply FCDA to this research, I use the discursive strategies of nomination and predication from DHA as well as van Leeuwen's representation of social actors to understand how Twitter users discursively construct women in the affinity space(s) of \#KickVic and \#IStandWithVic. With this analysis, I aim to answer the question: To what extent do these findings subvert or perpetuate dominant ideologies related to sexual harassment and these hashtags that tweeters use in the service of digital activism to address this social issue? The answer to this question is specifically attributed to the sexual harassment case of Mignogna and the findings produced from this analysis are not generalizable to every protest and counterprotest and instance of digital activism. 


\section{Chapter 4: Findings}

In the previous chapter, I explained the methodology of the approaches I am using (CL and CDS/FCDA), how I constructed the corpora for this project, and detail the procedures I undertake for the analysis. Presently, I discuss the findings from this analysis. The first section of this chapter covers the results of the word frequency analysis. Following this, I investigate the findings from the semantic theme and keyword analysis to demonstrate how the three corpora are similar and different with relation to a general reference corpus and with relation to each other. The final section of this chapter examines the term women through collocate and social actor analysis. Together, the results from these procedures show how the tweets discursively construct power asymmetries that subvert and perpetuate dominant ideologies.

\subsection{Corpora size differences}

Recalling the differing sizes of the three corpora I constructed, I argue that these totals are a finding as the corpus representing the counter-protest (\#IStandWithVic) contains nearly double the number of tweets compared to the corpus representing the protest (\#KickVic). By looking at the corpora breakdown according to year, I confirm that there are more \#IStandWithVic tweets from 2020 than there are \#KickVic: after I cleaned for retweets, 812 \#KickVic tweets contain 25,345 word tokens while 4,421 \#IStandWithVic tweets contain 119,847 word tokens. These totals mean that, from January 28,2020 -July 28,2020 , there were 3,609 more tweets hashtagged with \#IStandWithVic posted than those hashtagged with \#KickVic, resulting in a word token difference of 94,502. For comparison, the mixed corpus has 564 tweets from this period and these tweets consist of 19,119 word tokens. 
Furthermore, I observe a similar pattern in the corpora that combine the tweets from both years together: the \#KickVic corpus contains 7,720 tweets of 244,990 word tokens, the \#IStandWithVic corpus contains 14,497 tweets of 383,739 word tokens, and the mixed corpus contains 5,310 tweets of 171,514 word tokens. These totals make the \#KickVic corpus just over half as large as \#IStandWithVic, and this corpus has just under two-thirds of the total word tokens. Taking a closer look at the individual tweet and word token counts for 2019, I observe the following totals. Firstly, 6,908 \#KickVic tweets contain 219,645 word tokens while 10, 076 \#IStandWithVic tweets contain 263,892 word tokens. For comparison, the mixed corpus has 4,746 tweets from 2019 and these tweets consist of 152,395 word tokens.

From this knowledge, I determine a couple of findings. First, most of the word token totals (and similarly, tweets) come from the 2019 portion of the corpora. However, what is most interesting from the comparison is that even though the \#KickVic 2019 tweets are 3,168 fewer than \#IStandWithVic 2019, the word token counts of these sections have a substantial difference (a difference of 44,247). It could be possible that \#KickVic tweets from 2019 are composed of more tokens than those from \#IStandWithVic, owing to the lesser tweet number but similar word token count. Additionally, this difference is not due to a more restricted character limit present in 2019, as Twitter changed its limit from 140 to 280 in 2017. Future analysis could determine the average length of a tweet in each corpus to confirm this finding. However, as these findings relate to my research questions and the context of this protest and counter-protest, these data show that even though the counter-protest is bigger (in terms of size and frequency) it does not equate to success. Despite the \#KickVic corpus 
containing fewer tweets and occurring less frequently than the \#IStandWithVic hashtag from the same periods, \#KickVic's protest still achieved social change (having Mignogna removed from areas of the industry). Relating to this finding, I will discuss its implications in Chapter 4 and future directions of research concerning it in Chapter 5.

\subsection{Word frequencies}

The first word frequency finding I discuss is other frequently recurring hashtags in each of my three corpora. I examined the five most frequently recurring hashtags in each corpus and the number indicated in brackets after each hashtag is its raw frequency. In the \#KickVic corpus, frequent hashtags that tweeters use include \#standwithvic (138), \#metoo (74), \#iswv (72), \#fuckvic (62), and \#anime (60). For the \#IStandWithVic corpus, the top five frequent hashtags are \#vickicksback (769), \#iswv (488), \#westandwithvic (401), \#weebwars (297), and \#fearthebeard (262). Lastly, the five most frequent hashtags of the mixed corpus, other than \#KickVic and \#IStandWithVic, are \#vickicksback (590), \#vicmignogna (383), \#animegate (264), \#weebwars (210), and \#anime (170). Though my analysis does not attempt to investigate the complete network structure of these hashtags, these frequencies provide contextual information related to this protest and counterprotest concerning how the \#KickVic and \#IStandWithVic hashtags position themselves with other affinity spaces.

Secondly, the word frequency findings I address are from the difference in gendered language for words like he versus she, him versus her, his versus hers, he's versus she's, man versus woman, and men versus women across the three corpora. Even with the differences in corpus size considered, there are always more lexical items referring to a masculine person than a feminine person. Below are the raw frequencies for 
these gendered word tokens (Table 4) and normalized frequencies for the words across all three corpora (Table 5).

Table 4: Raw frequencies of gendered word tokens across corpora

\begin{tabular}{ccccccccccccc}
\hline & \multicolumn{1}{c}{ \#KickVic } & \multicolumn{4}{c}{ \#IStandWithVic } & \multicolumn{4}{c}{ Mixed } \\
\hline W & F & W & F & W & F & W & F & W & F & W & F \\
\hline he & 997 & she & 384 & he & 1679 & she & 708 & he & 436 & she & 244 \\
him & 803 & her & 326 & him & 1125 & her & 710 & him & 300 & her & 233 \\
& his & 732 & hers & 1 & his & 1039 & hers & 3 & his & 288 & hers & 1 \\
& he's & 313 & she's & 72 & he's & 244 & she's & 91 & he's & 102 & she's & 25 \\
& man & 245 & woman & 51 & man & 486 & woman & 91 & man & 140 & woman & 33 \\
& men & 39 & women & 152 & men & 60 & women & 120 & men & 21 & women & 39 \\
\hline Total & & 3129 & & 986 & & 4633 & & 1723 & & 1287 & & 575 \\
\hline
\end{tabular}

Note. $\mathrm{W}=$ Word, $\mathrm{F}=$ Frequency.

Table 5: Normalized frequencies of gendered word tokens across corpora

\begin{tabular}{ccccccccccccc}
\hline & \multicolumn{4}{c}{ \#KickVic } & \multicolumn{4}{c}{ \#IStandWithVic } & \multicolumn{4}{c}{ Mixed } \\
\hline W & F & W & F & W & F & W & F & W & F & W & F \\
\hline he & 41 & she & 16 & he & 44 & she & 18 & he & 25 & she & 14 \\
& him & 33 & her & 13 & him & 29 & her & 19 & him & 17 & her & 14 \\
& his & 30 & hers & 0 & his & 27 & hers & 0 & his & 17 & hers & 0 \\
& he's & 13 & she's & 3 & he's & 6 & she's & 2 & he's & 6 & she's & 1 \\
& man & 10 & woman & 2 & man & 13 & woman & 2 & man & 8 & woman & 2 \\
& men & 2 & women & 6 & men & 2 & women & 3 & men & 1 & women & 2 \\
\hline Total & & 129 & & 40 & & 121 & & 44 & & 74 & & 33 \\
\hline
\end{tabular}

Note. $\mathrm{W}=\mathrm{Word}, \mathrm{F}=$ Frequency. Normalized word frequencies were calculated per 10,000 words. Frequencies are rounded to the nearest one.

Although the raw frequencies across the corpora at first glance imply that masculine forms of the words are much more frequent than the female forms, the normalized word counts depict a different and more accurate finding. I observe from the totals of Table 5 that between the \#KickVic corpus and the \#IStandWithVic corpus, the frequencies of he, she, him, her, his and hers are similar to each other. For example, there are three fewer instances of he in the \#KickVic corpus than the \#IStandWithVic corpus. 
Likewise, there are two fewer instances of she in the latter corpus than the former. For all the sets of words across the three corpora, the masculine form of the word is more frequent than the feminine form. This finding recurs for all but one pair of words, men and women, as in all three corpora, the feminine form of the word is always more frequent than the masculine form. I argue that this evidence implies, in the case of women across all three corpora, that there is a stronger emphasis on the female collective, whereas for men, there is a more emphasis on the male individual. There is more evidence provided for this finding in the down-sampled analysis of the term women.

Of these gendered word tokens in Tables 4 and 5, seven of them are in the top 200 most frequent words in the \#KickVic corpus, six of them are in the top 200 in the \#IStandWithVic corpus, and six of them are in the top 200 in the mixed corpus. Table 6 shows the raw frequency rankings of the gendered word tokens by corpus. This rank order is significant because it illustrates another example of how these gendered word tokens compare within and across the corpora in terms of raw frequency. Although it is not definite who these pronouns refer to in all cases, the effect of this usage is that the hashtags \#KickVic and \#IStandWithVic appear to pertain more to discussions of men than women. Gendered word tokens referring to men consistently rank higher than gendered word tokens relating to women. Additionally, the lexical item man is higher ranked than either woman or women such that the former appears in the top 200 most frequent words of every corpus while the latter (woman and women) does not. This difference between the individual (man) and the collective (women) posed a potential avenue for an in-depth FCDA analysis of the discursive construction of women in the affinity space(s). I examine the findings of this analysis later in this chapter. 
Table 6: Rankings of gendered word tokens by corpus according to raw frequencies

\begin{tabular}{cccccc}
\hline \multicolumn{2}{c}{ \#KickVic } & \multicolumn{2}{c}{ \#IStandWithVic } & \multicolumn{2}{c}{ Mixed } \\
\hline Ranking & Word & Ranking & Word & Ranking & Word \\
\hline 38 & he & 33 & he & 57 & he \\
46 & him & 49 & him & 86 & him \\
51 & his & 54 & his & 89 & his \\
92 & she & 80 & her & 100 & she \\
105 & her & 81 & she & 104 & her \\
110 & he's & 116 & man & 167 & man \\
143 & man & & & & \\
\hline
\end{tabular}

\subsection{Keywords and semantic themes}

Because one portion of this project examines the similarities and differences between the three corpora, this section begins with an overview of their similarities before covering their differences. Thus, I first discuss the similarities of the three corpora (through semantic themes and keywords) from the comparison against the AmE06/BE06 and then explore the similarity findings from comparing the three corpora against one another. After this, I investigate the differences, following the same order as I used for the similarities. To address the concern for spelling variations (Section 3.7), I did not find that the generated keyword lists contained difficult to interpret variations and did not pose a problem during my analysis. For clarity, I provide general overviews from this analysis to illustrate the similarities and differences between the corpora. I encourage readers interested in examining the findings from every procedure I undertook from the enumerated list presented in Chapter 3 to consult Appendix C.

\subsubsection{Similarities}

After separating the tweets into corpora according to hashtags, the three corpora share several of their top 200 keywords when compared against the AmE06/BE06 (Table 7). Because of this, several of the semantic themes that I identified also recur across the 
corpora. Again, I categorized the keywords into semantic themes using collocates, concordance lines, and, when necessary, contextual information provided by the entire tweet (Section 3.8.3).

Table 7: AmE06/BE06 comparison: Shared across three corpora

\begin{tabular}{|c|c|c|}
\hline Semantic theme & All keywords of semantic theme & Total keywords \\
\hline References to Mignogna & $\begin{array}{l}\text { vic, mignogna, vic’s, @vicmignogna, } \\
\text { he’s }\end{array}$ & 5 \\
\hline Evidence & pic, evidence, proof, accusations, video & 5 \\
\hline Groups of people & $\begin{array}{l}\text { funimation,@funimation,@youtube, } \\
\text { fans,guys, people }\end{array}$ & 6 \\
\hline (Affinity) Spaces & con,twitter,@youtube & $3\left(2\right.$ unique $\left.^{2}\right)$ \\
\hline Attributes & innocent, guilty & 2 \\
\hline Pieces of web addresses & $\begin{array}{l}\text { youtube, @youtube, twitter, pic, www, } \\
\text { via, https, com, status, anime, youtu, co }\end{array}$ & 12 (9 unique) \\
\hline A collective & all & 1 \\
\hline Specified individuals & $\begin{array}{l}\text { individual_A }{ }^{3} \text {, funimation, } \\
\text { @ funimation, @youtube, anyone }\end{array}$ & 5 (2 unique) \\
\hline Actions & know, believe, want, think, prove, say & 6 \\
\hline
\end{tabular}

Of the eleven semantic themes that I identify, the three corpora share nine of them. Two semantic themes - protest and harassment - are only shared between the \#KickVic corpus and the mixed corpus (Appendix C, Table C.1.2). Some of the keywords I considered to be a part of more than one semantic theme. For example, Funimation, as a company, is a group of people and a specified individual. From these semantic themes, the pieces of web address theme has the most amount of keywords (twelve), and the semantic theme of a collective has the least amount (one). Additionally, the semantic themes of references

\footnotetext{
${ }^{2}$ Some keywords belong to more than one semantic theme. For determining totals, 'unique' means that the number of keywords in brackets has not yet been categorized under a semantic theme.

${ }^{3}$ This is a pseudonym. To protect user identities, I use the naming convention individual_A, individual_B, etc., to refer to these individuals.
} 
to Mignogna (five keywords), groups of people (six keywords), and specified individuals (five keywords) all have a similar number of ways in which tweeters referred to the theme. Of the specified individuals, the three corpora all refer to one person by name, and all other keywords of this semantic theme are entities and a pronoun. All three corpora refer to the attributes innocent and guilty. Furthermore, all three corpora share many words for the semantic themes evidence (five keywords) and actions (six keywords). For the theme evidence, the highest total of keywords I identified comes from the similarities between the \#KickVic corpus and the mixed corpus similarity (six keywords; see Appendix C, Table C.1.2). Likewise, the highest number of keywords related to the actions semantic theme is seven, identified in the \#KickVic corpus and mixed corpus comparison (Table C.1.2) and the \#IStandWithVic corpus and mixed corpus comparison (Table C.1.3). Lastly, I establish the semantic theme (affinity) spaces and it references both physical and digital gatherings of people. The three corpora share three keywords for this semantic theme. Not including keywords that fit into more than one semantic theme, the three corpora share thirty-eight keywords out of two hundred (Table 7).

When I examined the similar semantic themes and keywords from the pairs comparison, the results illustrated another aspect of these corpora. Firstly, the \#KickVic and \#IStandWithVic corpora share the same nine semantic themes as all three corpora when I compared them against the AmE06/BE06 wordlists (Table C.1.1). Additionally, new keywords belong to these themes. Not counting keywords that belong to more than one theme, these corpora share forty-one keywords out of two hundred. Next, examining the similarities between the \#KickVic corpus and mixed corpus, this is the only comparison against the AmE06/BE06 where I found all eleven semantic themes that I 
identified. Not counting keywords that fall under more than one semantic theme, the \#KickVic corpus and mixed corpus share fifty-one keywords out of two hundred (Table C.1.2). These findings illustrate that the hashtags \#KickVic and \#IStandWithVic are not necessarily as divergent as we might assume because these hashtags appear to index a similar set of semantic themes. This finding recurs in the similarities between the \#IStandWithVic corpus and the mixed corpus when I compared them against the AmE06/BE06. Like the comparison of all three corpora and the \#KickVic corpus and \#IStandWithVic corpus, these two corpora share the same nine semantic themes. However, I categorized several new keywords under these semantic themes. Not including keywords that belong to more than one theme, the \#IStandWithVic corpus and the mixed corpus share fifty-three keywords of two hundred (Table C.1.3). This finding supports the idea that these corpora share the most keywords, indicating that of the three corpora, these two are the most similar in terms of the keywords that do occur. Again, this suggests that oppositional hashtags of a protest and counter-protest are not necessarily as divergent as we might assume because these comparisons show that the corpora index similar semantic themes. I discuss the interpretation of this finding in Chapter 4.

These findings represent how similar the three corpora are when comparing the shared keywords (both taken as a set of three and taken in pairs). Additionally, the results provide supporting evidence regarding how they are different from each other. First, a reminder of how many similar keywords there are for each comparison. All three corpora share thirty-eight keywords, and the \#KickVic corpus and the \#IStandWithVic corpus share forty-one keywords. Additionally, the \#KickVic corpus and the mixed corpus share 
fifty-one keywords and the \#IStandWithVic corpus and the mixed corpus share fifty-three keywords.

As I suggested, the \#IStandWithVic corpus and the mixed corpus are the most similar concerning the keywords of each corpus as they share the most keywords (fiftythree). Taken together, the comparisons between the \#KickVic corpus/mixed corpus and the \#IStandWithVic corpus/mixed corpus show a higher number of shared keywords than those found in the \#KickVic corpus and \#IStandWithVic corpus. From this evidence, I argue that the \#KickVic corpus and the \#IStandWithVic corpus are more like the mixed corpus than they are to each other in consideration of the keywords that occur in the corpora (fifty-one, fifty-three, and forty-one, respectfully). This finding is significant because it illustrates that the mixed corpus reflects a mixture of politically opposite stances of the two hashtags. However, that is not to say that using the two hashtags in one tweet always indicates common ground. Instead, as the down-sampled analysis of women will show, a tweeter might invoke one hashtag in a tweet to refer to one side of the protest while the other indicates the Twitter user's stance.

However, I determined these similarities with the AmE06/BE06, where I generated the keywords using general written American and British English. To understand the similarities of the corpora compared to the contents of the corpora, I directly compare each corpus against the others. Though this resulted in less than 200 keywords for each comparison due to the size of the corpora, it is still possible to categorize the shared keywords into semantic themes. When compared directly against each other, I found no keywords shared by all three corpora. However, this finding is a result of the procedure because it automatically rules out any similarities. Due to this, the 
remainder of the similarities section considers an overview of the results when I examined the corpora in pairs.

Examining the corpora in sets of two, the \#KickVic corpus and the \#IStandWithVic corpus only share four keywords when I compared each against the mixed corpus (Appendix C, Table C.1.4). With this said, \#KickVic only has forty-one keywords when compared against this corpus, and \#IStandWithVic has seventy-five keywords total. All four of these keywords are pronouns (he, him, his, and you), and after examining concordance lines, I determined the most common referent for he, him, and his is Mignogna. Also, I previously discovered that against a general reference point, the keywords of \#KickVic and \#IStandWithVic are the least like each other than what exists between other comparisons. Given that the \#KickVic corpus and the \#IStandWithVic corpus are like the mixed corpus and different from each other, I believe it makes sense that they do not share many keywords. Lastly, with the similar keywords, I think this signals that the main talking point of these corpora is Mignogna. For more details on this finding, see Appendix C.

Secondly, I examined the similarities between the \#KickVic corpus and the mixed corpus when the \#IStandWithVic corpus is the reference corpus (Table C.1.5). Of the eleven previously identified semantic themes, these two corpora share six of them. Altogether, these corpora share fifteen keywords in this comparison. Lastly, I examined the shared semantic themes between the \#IStandWithVic corpus and the mixed corpus with the \#KickVic corpus as the reference corpus (Table C.1.6). These corpora share two different semantic themes than the \#KickVic corpus and the mixed corpus do, for a total 
of six shared semantic themes. Not including keywords that are doubles, the \#IStandWithVic corpus and the mixed corpus share thirty-two keywords.

As this analysis shows, specific corpora share more similarities with one than another. Firstly, all three corpora shared zero keywords and the \#KickVic corpus and the \#IStandWithVic corpus against the mixed corpus shared four keywords. Additionally, the \#KickVic corpus and the mixed corpus against the \#IStandWithVic corpus shared fifteen keywords. Lastly, the \#IStandWithVic corpus and the mixed corpus against the \#KickVic corpus shared thirty-two keywords. From these totals, a few patterns merit comment. As previously stated, the \#KickVic corpus and \#IStandWithVic corpus are more like the mixed corpus than they are to each other. Because of this similarity, I believe this results in few shared keywords between them when compared directly against the mixed corpus. Additionally, the \#IStandWithVic corpus and the mixed corpus against the \#KickVic corpus share the most keywords of these sets of comparisons. Though the \#KickVic corpus and the mixed corpus against the \#IStandWithVic corpus share more keywords than the \#KickVic corpus and the \#IStandWithVic corpus against the mixed corpus comparison, these corpora share less than half of the total keywords (fifteen) than the \#IStandWithVic corpus and the mixed corpus share (thirty-two). These results imply that the \#IStandWithVic corpus and mixed corpus have more in common with each other in terms of keywords than the \#KickVic corpus and mixed corpus do. Also, this finding repeats, as in the set of comparisons against the AmE06/BE06, the \#IStandWithVic corpus and mixed corpus not only shared the most keywords but shared more keywords than the \#KickVic corpus and the mixed corpus did. As I observe in Section 4.4, this similarity finding between the \#IStandWithVic corpus and mixed corpus will reoccur. 
This finding strongly implies that Twitter users who engage with the counter-protest adopt counter-protest hashtags and the combination of hashtags as a discursive construction to perpetuate their stance towards the social issue of sexual harassment. With the analysis of similarities complete, I discuss what makes each corpus unique in comparison to the AmE06/BE06 and the other corpora.

\subsubsection{Differences}

Repeating the structure from the previous section, I first provide a generalized overview of the keywords unique to each corpus when compared against the AmE06/BE06. After this, I summarize the keywords unique to each corpus when compared against each other. For reasons of clarity, the semantic themes of this section are slightly different from those determined when I examined the corpora for similarities. Furthermore, I combined the semantic themes actions and attributes into one theme (actions and attributes) and groups of people and specified individuals into one theme (people). Also, I have added the categories discourse, to encompass those keywords that mean instances of language, and other, for keywords that do not comprise one semantic theme.

Investigating first what makes the \#KickVic corpus unique when compared against the AmE06/BE06, I identified four semantic themes and fourteen unique keywords (Appendix C, Table C.2.1). For this corpus, three unique keywords refer to (affinity) spaces, and one of these is a hashtag unique to this corpus (\#standwithvic). This hashtag as a keyword in this corpus provides supporting evidence that these corpora consist of tweets that are not only of the same stance as the hashtag. To clarify, even though this is the \#KickVic corpus, tweets related to the \#IStandWithVic affinity space are 
a part of it. Additionally, this finding exemplifies the fluidity, also understood as intertextuality and dialogism, between these connected hashtags, and shows that the discourses of both the \#KickVic corpus and \#IStandWithVic corpus are inherently related. Furthermore, this fluidity (in terms of other hashtags this study has not investigated) speaks to the hijacking strategy scholars have previously recognized (Barker-Plummer \& Barker-Plummer, 2017; Gallagher et al., 2018). I discuss the implications of this finding concerning the research questions in Chapter 5.

Exploring the unique keywords to the \#IStandWithVic corpus when compared against the AmE06/BE06 next, the differences between this corpus and the \#KickVic corpus are made clear (Table C.2.2). For example, the \#KickVic corpus contains unique keywords related to (affinity) spaces that describe these spaces generally (movement and hashtag). However, the keywords of this semantic theme for the \#IStandWithVic corpus all specify the content of the space. Additionally, the four keywords are hashtags unique to this corpus (\#women4individual_F, \#women4vic, and \#women) or refer to the protest $(k v)$. There is also a difference between these two corpora in that for this comparison, the \#IStandWithVic corpus does not contain any unique keywords related to the semantic theme harassment. As we will see later with the direct corpus comparison, harassment is categorized differently in this corpus. Another difference between these two corpora is that the \#IStandWithVic corpus contains many more unique keywords of the semantic theme actions and attributes (eight keywords). Of these semantic theme's eight keywords, five denote positive feelings and support towards an individual (love, support, stand, hope, and thank), one is a mental process (see), and two are material processes that denote negativity (kicks and blocked). Lastly, the \#IStandWithVic corpus contains ten 
unique keywords that indicate people whereas the \#KickVic corpus only had three. Of these keywords, seven refer to specific individuals, and one of them references an anime character that Mignogna performed the voice for (broly). Altogether, the \#IStandWithVic corpus contains twenty-two unique keywords when compared against the AmE06/BE06.

Lastly, the number of unique keywords that the mixed corpus contains is similar to the total of the \#KickVic corpus (Table C.2.3). This corpus has fourteen unique keywords, not including doubles, when compared against the AmE06/BE06. I categorized these keywords into five semantic themes. Like the \#IStandWithVic corpus, the keywords of the semantic theme (affinity) spaces are all hashtags for the mixed corpus. As well, this corpus nominates unique individuals or groups when referencing people. This finding patterns closer to the \#IStandWithVic corpus than the \#KickVic corpus because the latter only contained one unique keyword that nominated an individual. Additionally, keywords of this corpus include vs (versus) and sides, which I believe is related to the fact that tweets in this corpus contain both the hashtags \#KickVic and \#IStandWithVic. In my view, this finding indicates that a characteristic of the mixed corpus is the existence of the two hashtags battling for dominance. However, during this portion of the analysis, it is unclear how Twitter users discursively construct this struggle (see: Section 4.4).

From the comparison against the AmE06/BE06, I discovered that the \#IStandWithVic corpus has the most unique keywords (twenty-two), whereas the \#KickVic corpus and the mixed corpus still do have unique keywords but a smaller amount of them (fourteen words each). Though I believe the number of unique keywords to \#IStandWithVic is attributed to the corpus' size, I find it interesting that the unique 
keywords of this corpus and the mixed corpus are more similar in terms of semantic themes. For the semantic themes (affinity) spaces and people, these corpora have many more unique keywords that are hashtags and nominated social actors than the \#KickVic corpus does for the same category. Though more evidence is needed, I believe this finding suggests that the counter-protest references more (affinity) spaces to perpetuate their ideology to more Twitter users and establish it as the social norm. Lastly, while the \#IStandWithVic corpus and the mixed corpus are more like each other when compared to a corpus of general written Englishes, the direct corpus comparison to follow shows that the \#KickVic corpus shares these similarities too.

The final step required to answer the research question of how these three corpora are similar and different is to explore the unique keywords of each corpus generated by directly comparing the corpora to one another. I believe these findings to be some of the most interesting as it shows what makes each corpus unique in terms of the discursive constructions of the protest and counter-protest. Firstly, the \#KickVic corpus contains forty-nine unique keywords, not counting words that fit more than one category, belonging to six semantic themes when compared directly against the other corpora (Appendix C, Table C.2.4). A few of these unique keywords are the same as when I generated its keywords against the AmE06/BE06 (claims and assault), providing further evidence that these keywords are unique to this corpus. Given that people started using this hashtag (\#KickVic) to ambiently affiliate around the topic of instances where Mignogna sexually harassed others in the past, I believe these keywords reflect these origins. Additionally, this corpus uniquely refers to the protest as a cause and a campaign, rather than some of the other unique keywords that belong to the other corpora 
and frame this event differently. Lastly, seventeen keywords belong to the semantic theme actions and attributes. Three of these keywords have a negative connotation (spooky, unwanted, gross, shit) and tweeters use others to reference the act of reporting sexual harassment (many, recount, caught, defending, and enough). As I will observe, the keywords of the theme actions and attributes discursively construct the \#KickVic corpus and the \#IStandWithVic corpus very differently, once again reaffirming the finding that these two corpora are the least like each other concerning the stance of the tweeters with relation to the topic of sexual harassment.

Secondly, I examine unique keywords to the \#IStandWithVic corpus. Not counting keywords that belong to more than one semantic theme, I found thirty-nine unique keywords, nine fewer unique keywords than what exists in the \#KickVic corpus (Table C.2.5). Whereas the AmE06/BE06 comparison did not result in any unique keywords of the semantic theme harassment, the direct corpus comparison shows that the \#IStandWithVic corpus has one word related to this theme (hug). Another finding from these unique keywords is that the keywords belonging to the semantic themes of the protest and actions and attributes frame the corpora very differently. While users construct the protest in the \#KickVic corpus as a cause and campaign, the unique keyword associated with this theme for the \#IStandWithVic corpus is petition (to change the outcome of the protest). Contrastively, the \#KickVic corpus constructs the protest as an event that insights change and the \#IStandWithVic corpus constructs it as an event that people need to oppose. Furthermore, while the unique keywords of the \#KickVic corpus relating to actions and attributes discursively construct this theme as about sexual harassment, the unique keywords of the \#IStandWithVic corpus tell a different story. This 
semantic theme contains fifteen unique keywords, many of which construct positive sentiment (wonderful, good, awesome, beautiful, and positivity) and supportive actions (hug, donate, please, pray, rehire, and stay). Taken together, this constructs the actions and attributes of the \#IStandWithVic corpus as supporting Mignogna, rather than about harassment like the unique keywords from the \#KickVic corpus shows.

Lastly, I identified seventeen keywords unique to the mixed corpus (not including those that fit into more than one theme) and these keywords belong to five different categories (Table C.2.6). This corpus has the lowest number of unique keywords, though I believe this is because, as I previously discovered, that this corpus has many similarities to the other corpora. Firstly, all the keywords belonging to the semantic theme (affinity) spaces are hashtags. Not only were all the keywords related to this theme also hashtags when I compared the mixed corpus against the AmE06/BE06, but all the unique keywords of this theme for the \#IStandWithVic corpus are hashtags as well. This result reinforces the finding that the mixed corpus and the \#IStandWithVic are like each other. Also, the mixed corpus refers to harassment with the unique keywords \#evidencematters, attacks, and controversy. Likewise, tweeters construct the protest semantic theme with the keywords war and controversy. Recalling how the other two corpora construct the protest (as an event that insights change and as an event that needs to be opposed), this finding shows that the mixed corpus constructs this semantic theme differently. Rather than the protest as an event to be proven incorrect, the mixed corpus constructs it as a conflict between different groups (war) due to disagreement between those groups (controversy). This idea is further supported by the finding that this corpus contains both 
hashtags, and as such, it is not a surprise that it views the protest from more than one angle.

With these findings, it is possible to answer one research question: Are the semantic themes similar and/or different in the opposing hashtags? First, I will summarize the unique keywords found by directly comparing the corpora against each other. As a reminder, the \#KickVic corpus has forty-eight unique keywords, the \#IStandWithVic corpus has thirty-nine unique keywords, and the mixed corpus has seventeen unique keywords. In contrast to the keywords for each corpus when compared against the AmE06/BE06, the \#KickVic corpus now contains the most unique keywords. For the comparison against the other reference corpora, the \#IStandWithVic corpus contained the most unique keywords (twenty-two keywords). I believe these findings support two ideas. Firstly, the \#IStandWithVic corpus is the most unique of the three corpora when compared against general written Englishes. Secondly, the \#KickVic corpus is the most unique when I analyzed the three corpora against each other. Furthermore, this result corroborates the finding that the \#IStandWithVic corpus and the mixed corpus are the most similar. Additionally, the corpora share many of the semantic themes I identified; what makes the corpora unique are the keywords associated with these semantic themes. As such, I argue that the three corpora talk about the same issues (semantic themes), but how tweeters who use the \#KickVic and \#IStandWithVic hashtags discursively construct these issues differs at the lexical level. For example, each corpus has keywords related to the themes (affinity) spaces, actions and attributes, and people. This finding stresses why the synergy between $\mathrm{CL}$ and CDS/FCDA is a central component of this project because the CL procedures alone cannot access how tweeters 
discursively construct these issues differently. For reference, Table 8 below summarizes the number of unique keywords for each corpus from both comparisons. As well, I list which corpora contain which semantic themes I identified.

Table 8: Totals of unique keywords for each corpus

\begin{tabular}{ccc}
\hline & AmE06/BE06 comparison & Direct corpus comparison \\
\hline \#KickVic & 14 & 48 \\
\#IStandWithVic & 22 & 39 \\
Mixed & 14 & 17 \\
\hline
\end{tabular}

1. AmE06/BE06 comparison

a. \#KickVic corpus semantic themes: (affinity) spaces, harassment, actions and attributes, people

b. \#IStandWithVic corpus semantic themes: (affinity) spaces, actions and attributes, people

c. Mixed corpus semantic themes: (affinity) spaces, discourse, the protest, actions and attributes, people

2. Direct corpus comparison

a. \#KickVic corpus semantic themes: (affinity) spaces, harassment, the protest, actions and attributes, people, other

b. HIStandWithVic corpus semantic themes: (affinity) spaces, harassment, the protest, actions and attributes, people, other

c. Mixed corpus semantic themes: (affinity) spaces, harassment, the protest, actions and attributes, people

Knowing what makes these corpora unique and how they are unique compared to each other, I also better understand their similarities. Firstly, the corpora share many semantic 
themes, but the number of keywords associated with these themes and the kinds of keywords belonging to themes varies between corpora. Against the AmE06/BE06, the \#IStandWithVic corpus and the mixed corpus share the most keywords (fifty-three). Contrastively, the \#KickVic corpus and the mixed corpus share the most semantic themes (eleven in total). Likewise, for the direct corpus comparison, the \#IStandWithVic corpus and the mixed corpus share the most keywords (thirty-two). Unlike the other comparison, both the \#KickVic corpus/the mixed corpus and the \#IStandWithVic corpus/the mixed corpus share the same number of semantic themes (six in total). The above indicates a few findings, especially in consideration of the unique keywords results. As previously stated, the \#KickVic corpus and the \#IStandWithVic corpus are the least similar. Also, the \#IStandWithVic corpus and the mixed corpus are the most like each other. Additionally, the \#KickVic corpus and the \#IStandWithVic corpus are more like the mixed corpus than each other, and the \#IStandWithVic corpus is more like the mixed corpus than the \#KickVic corpus is. Due to this, I argue that these results reinforce the finding that the \#IStandWithVic corpus and the mixed corpus are the most similar of the three corpora and the \#KickVic corpus is the most different of the three corpora. For reference, Table 9 below summarizes the number of similar keywords for each pair of corpora from both comparisons. As well, I list which corpora contain which semantic themes I identified.

Table 9: Totals of similar keywords for each corpus comparison

\begin{tabular}{ccc}
\hline & AmE06/BE06 comparison & Direct corpus comparison \\
\hline All three corpora & 38 & 0 \\
\#KickVic and & 41 & 4 \\
\#IStandWithVic & & \\
\#KickVic and mixed & 51 & 15 \\
\#IStandWithVic and & 53 & 32 \\
mixed & & \\
\hline
\end{tabular}




\section{AmE06/BE06 comparison}

a. All three corpora semantic themes: references to Mignogna, evidence, (affinity) spaces, groups of people, attributes, pieces of web addresses, a collective, specified individuals, actions

b. \#KickVic corpus and \#IStandWithVic corpus semantic themes: references to Mignogna, evidence, (affinity) spaces, groups of people, the protest, attributes, pieces of web addresses, a collective, specified individuals, actions

c. \#KickVic corpus and mixed corpus semantic themes: references to Mignogna, evidence, (affinity) spaces, groups of people, the protest, attributes, harassment, pieces of web addresses, a collective, specified individuals, actions

d. \#IStandWithVic corpus and mixed corpus semantic themes: references to Mignogna, evidence, (affinity) spaces, groups of people, attributes, pieces of web addresses, a collective, specified individuals, actions

2. Direct corpus comparison

e. All three corpora semantic themes: none

f. \#KickVic corpus and \#IStandWithVic corpus semantic themes: pronouns

g. \#KickVic corpus and mixed corpus semantic themes: (affinity) spaces, groups of people, the protest, a collective, specified individuals, actions

h. \#IStandWithVic corpus and mixed corpus semantic themes: references to Mignogna, (affinity) spaces, groups of people, pieces of web addresses, specified individuals, actions 


\subsection{Down-sampling of women}

Even though these hashtags are about what happens when women address unspoken instances of sexual harassment to initiate social change, I found it interesting that the lexical items woman and women were not in the top 200 keywords for any of the corpora when compared against the AmE06/BE06 frequency wordlists. However, when I examined the \#KickVic corpus against the \#IStandWithVic corpus and the mixed corpus separately, I discovered that the lexical item women was a keyword (keyness of +31.73 and 37.19 , respectively) in only this corpus. The lexical item victims (a keyword) is also a keyword of the \#KickVic corpus, though it is shared by the \#KickVic corpus and the mixed corpus when I separately used the \#IStandWithVic corpus as the reference corpus (Appendix C.1, Table C.1.5). When I analyzed the collocates and concordance lines of women and victims, I noticed words that Palomino-Manjón (2020) has also identified as frequent collocates of (sexually) violent acts and social actors, like believe, men, people, sexual, support, and victims. Considering these previously identified recurring linguistic, I decided not to follow the corpus in terms of high frequency words and keywords to investigate this contextually relevant social actor and how, given its low frequency, tweeters discursively construct women in this protest and counter-protest. To understand how Twitter users across the corpora discursively construct this actor, the subsequent sections detail my findings from the in-depth women collocation and social actor analysis.

\subsubsection{Collocation analysis}

With the frequencies of women and woman in the corpora accounted for, it is best to discuss the findings from the collocate analysis. I examined the singular and plural 
forms separately because the collective term versus the individual may provide insights into how tweeters discursively construct this social actor regarding assimilation and individualization. I concentrate on the difference between women and woman; below are the top ten collocates for these words in the three corpora, ranked according to their statistical significance using log-likelihood. I chose the top ten collocates because each corpus had at least ten collocates for each word (Tables 10 and 11).

Table 10: Collocates for women across corpora

\begin{tabular}{|c|c|c|c|c|c|c|c|c|}
\hline \multicolumn{3}{|c|}{ \#KickVic } & \multicolumn{3}{|c|}{ \#IStandWithVic } & \multicolumn{3}{|c|}{ Mixed } \\
\hline $\mathrm{F}$ & MI & $\mathrm{C}$ & $\mathrm{F}$ & $\mathrm{MI}$ & $\mathrm{C}$ & $\mathrm{F}$ & MI & $\mathrm{C}$ \\
\hline 9 & 8.26977 & young & 6 & 8.32095 & men & 8 & 9.81716 & women \\
\hline 7 & 8.17639 & men & 10 & 8.05791 & women & 6 & 8.66516 & lie \\
\hline 5 & 5.31815 & where & 5 & 8.03407 & speak & 5 & 8.5176 & always \\
\hline 11 & 5.22817 & these & 5 & 6.95358 & victims & 7 & 8.00303 & hate \\
\hline 6 & 5.12046 & sexual & 11 & 6.3144 & believe & 8 & 6.38832 & can \\
\hline 5 & 4.93744 & support & 5 & 6.18344 & hate & 5 & 4.60751 & not \\
\hline 7 & 4.93431 & believe & 12 & 5.94707 & these & 6 & 4.43604 & in \\
\hline 17 & 4.6785 & who & 5 & 5.48099 & over & 15 & 4.24915 & and \\
\hline 17 & 4.6785 & about & 5 & 5.37609 & side & 5 & 4.12643 & are \\
\hline 8 & 4.43769 & know & 26 & 5.18218 & are & 8 & 3.91398 & of \\
\hline
\end{tabular}

Note. $\mathrm{F}=$ Frequency, $\mathrm{M}=\mathrm{MI}$ statistical value, $\mathrm{C}=$ Collocate .

Table 11: Collocates for woman across corpora

\begin{tabular}{ccccccccc}
\hline & \#KickVic & \multicolumn{3}{c}{ \#IStandWithVic } & \multicolumn{3}{c}{ Mixed } \\
\hline $\mathrm{F}$ & MI & C & F & MI & C & F & MI & C \\
\hline 13 & 5.86698 & who & 14 & 6.10786 & i'm & 5 & 6.73476 & she \\
32 & 5.13125 & a & 6 & 6.06851 & said & 21 & 5.47438 & a \\
5 & 4.45779 & as & 7 & 5.92451 & man & 5 & 4.84852 & not \\
13 & 4.12141 & is & 5 & 5.83252 & am & 6 & 4.59314 & for \\
15 & 3.77297 & and & 8 & 5.57437 & she & 8 & 4.43143 & this \\
8 & 3.68015 & that & 13 & 5.5052 & who & 6 & 4.22204 & that \\
5 & 3.64272 & are & 55 & 5.28369 & a & 6 & 3.70997 & is \\
18 & 3.53065 & the & 7 & 5.02596 & because & 8 & 3.58327 & and \\
6 & 3.50145 & this & 5 & 4.89222 & her & 5 & 3.2845 & i \\
7 & 3.17504 & you & 6 & 4.80039 & up & 9 & 3.16671 & \#kickvic \\
\hline
\end{tabular}

Note. $\mathrm{F}=$ Frequency, $\mathrm{S}=$ MI statistical value, $\mathrm{C}=$ Collocate. 
From these collocates, a few interesting patterns emerge. Of the content words for women (those that have semantic content), men and believe occur in the top ten collocates of the \#KickVic and \#IStandWithVic corpora. Additionally, women and hate appear on the collocate list for the \#IStandWithVic and mixed corpora. There is not such a pattern for the collocates of woman across the corpora, though it is important to remember that this word is less frequent than its plural counterpart and therefore would have a lower number of statistically significant (MI score > 3.0) collocates that are also content words.

Examining all the collocates for woman, very few are content words, all of which are topranked according to MI score. The collocates for women pattern similarly except that some content words are not in the top ten most statistically significant. Those collocates that are not in Table 5 are listed below (MI score indicated in brackets):

- \#KickVic corpus: vic (3.5698)

- \#IStandWithVic corpus: think (5.04793), man (5.03999), people (3.4547)

Furthermore, the singular form man is more frequent in each corpus (Tables 4 and 5), but the plural form of men collocates with women in two of the three corpora. As well, men is ranked as the second and first statistically significant collocate in the \#KickVic corpus and \#IStandWithVic corpus. Another finding from these collocates is that in \#IStandWithVic and the mixed corpus, women is a collocate of women. Additionally, women ranks very highly in terms of its significance in the \#IStandWithVic corpus and the mixed corpus. I never encountered a word being a collocate of itself, and upon closer inspection of concordance lines, I discovered that this finding is not odd, especially given the type of data I am analyzing. For example, sometimes the tweeter would use the word women in the tweet and then use a hashtag that contains women (Example 1). 
Example $1^{4}$ : Saw one photo of me fully clothed and yet Im reduced to being a thirst trap. [hashtags removed] but they claim to be the pro women side, unless of course women disagrees with them. (-) \#Womenforvic \#IstandwithVic [hyperlink removed]

Another instance includes a URL that contains the word women in it twice. Lastly, instead of replacing the noun women with a pronoun, some users chose to refer to the subject or object of their tweet a second time by repeating the word women (Example 2).

Example 2: I don't follow the "believe all women" cause I know women can lie. I had a friend who was falsely accused of sexual assualt by a girl. So I learned that not all women tell the truth. \#IStandWithVic

I observe from the above example that tweeters construct women as a passive object and the agent of a harmful action (the possibility of lying). I consider the usage of women as the subject and object in these corpora in Chapter 4 for what it reveals about how tweeters discursively construct women in this protest and counter-protest.

Verb collocates prove to be particularly meaningful in these three corpora too. Verbs represent the action that a subject performs or the action that the agent does to an object. In my findings, collocate verbs of women are actions that women perform (or that tweeters say women perform) or are actions that others do to women (or that tweeters say are done to women). The following verbs collocate with women in the specified corpora

\footnotetext{
${ }^{4}$ Each example I use from the corpora I quote verbatim; as such, there are misspellings in the text. As well, I removed text not necessary for interpretation from all examples to protect user identities (Section 3.6).
} 
(the first number for each collocate represents frequency, the second represents MI score):

- \#KickVic corpus: support (5, 4.93744), believe (7, 4.93431), know (8, 4.43769)

- \#IStandWithVic corpus: speak $(5,8.03407)$, believe $(11,6.3144)$, hate (5, 6.18344), think $(6,5.04793)$

- $\quad$ Mixed corpus: lie $(6,8.66516)$, hate $(7,8.00303)$

Initially, I may be inclined to assume that the collocate verbs in \#KickVic are helpful to women, the ones in \#IStandWithVic are both helpful and harmful, and the ones in the Mixed corpus are harmful. However, I must consider the usage and meanings of these verbs in context (i.e., concordance lines) to understand how they relate to the noun women. First, I analyzed how tweeters use the verbs support, believe, and know in the \#KickVic corpus using the concordance lines attributed to the collocates. As well, at times, I had to consult the entire tweets to discern the meaning of the verbs. For the verb support, all but one instance references supporting women or how supporting Mignogna results in the failure of support for women (Example 3).

Example 3: I'm blocking people supporting Vic, you all are nasty motherfuckers and are a failure to sexual assault survivors. Ppl who support Vic fail women and young women alike. PPL WHO SUPPORT VIC FAIL THE SAFETY OF THE PUBLIC!

I HAVE 0 TIME FOR YOUR DELUSIONAL NONSENSE! \#KickVic

Examining the verb believe, one of the instances of this collocate is due to a URL that contains both the words believe and women. Two instances of this collocate are attributed 
to users saying that women need to be believed when they come forward about sexual harassment. Another occurrence remarks that it should be easier to believe that Mignogna sexually harassed women than a fabricated conspiracy that the industry created to fire Mignogna. Lastly, two examples of this collocate where users clarify that it is not that they do not believe women but that they would rather believe facts and evidence (Example 4).

Example 4: In case you pull out this card, I'm not sayin don't believe all women. I'm sayin that you should use your brain to determine whether that person is trustworthy or not, by looking at evidence, which is something \#KickVic have not done.

For the final verb know, users know of women and therefore know they are not lying. Though all the examples refer to knowing that women have been sexually harassed, all but one example attributes this knowledge in a helpful way to the \#KickVic movement. As such, only one tweeter uses this knowledge in a way to delegitimize the movement. Looking at the verb collocates in \#IStandWithVic, speak is always used in the construction [negated auxiliary verb] speak for all women. Tweeters imply in their tweets that those on the side of \#KickVic speak for all women. They distance themselves from \#KickVic with this construction and state that they believe in justice which requires evidence. The verb believe in this corpus occurs four out of nine times in quotation marks with the noun women. Seven of the nine times women are the object of believe. In one occurrence, women are believing, and the last instance of this collocate is a hashtag that includes the word women and believe. Lastly, four of these instances echo sentiments that 
users do not believe women because women can lie (Example 2) or the tweeters believe the evidence (Example 5).

Example 5: No you are wrong buddy it's not that we don't believe women we believe facts and evidence and so far there is none, but lots of evidence of false claims. So until I see real evidence drop \#IStandWithVic

For the verb hate, women are always the object of the action. These users argue that their position (\#IStandWithVic) does not indicate that they hate women. However, one user states that they hate women because women "wrongly accused" Mignogna with no proof. Overall, tweeters used many instances of these verbs to depict women in a harmful manner.

The mixed corpus has two collocates of women that are verbs: lie and hate. In three of these instances, women are the subject of lying (Example 6). One of these instances poses the possibility of women lying through the modal auxiliary can (Example 7). Two other instances of this collocate dispute why women would lie (Examples 6 and $8)$.

Example 6: \#kickvic \#istandwithvic women do not lie right? Is it fair to give them the power to ruin someone's life based on their words alone?

[hyperlink removed]

Example 7: Women can lie

Women can manipulate

Women can abuse

So why don't we call out these kind of bitches out the same way we're doing to men like this online? 
\#istandwithvic \#KickVic [hashtag removed]

Example 8: \#kickvic Why are you guys constantly asking the question, "Why would women lie about assault?" Well my question to you is why are you guys lying to run this false narrative about Vic?? \#istandwithvic

Of the four examples of this collocate, women are not the actor of lying or the object undergoing the lying. Instead, the phrase harass women is conjoined to lie as two separate actions that a social actor performs. Lastly, there is the word hate, and of the seven occurrences of this verb, women are the object of this action. In one example of this word, hate is a noun rather than a verb and is used to describe a group that hates women (Example 9).

Example 9: Oh they are lol. They assume it is all males and it's a women hate group guess what \#istandwithvic and \#kickvic guess I am a trader to my gender as I am tired of feminist agenda. All for female rights but geez do they over kill it.

As such, in the mixed corpus, I observe that women are more frequently the actor of lying than the object, but they are only ever the object of hatred rather than enacting this action. To summarize the collocate results, I provide the three tables below.

Table 12: Totals of women as actors and objects in the \#KickVic corpus

\begin{tabular}{ccccccccccc}
\hline & \multicolumn{3}{c}{ Support (5) } & \multicolumn{3}{c}{ Believe (7) } & \multicolumn{3}{c}{ Know (8) } \\
\hline & Actor & Object & Other & Actor & Object & Other & Actor & Object & Other \\
\hline Women & 0 & 2 & 3 & 0 & 5 & 2 & 0 & 4 & 4 \\
\hline
\end{tabular}


Table 13: Totals of women as actors and objects in the \#IStandWithVic corpus

\begin{tabular}{cccccccccccc}
\hline & \multicolumn{3}{c}{ Speak (5) } & \multicolumn{3}{c}{ Believe (11) } & \multicolumn{3}{c}{ Hate (5) } & \multicolumn{2}{c}{ Think (6) } \\
\hline & Actor & Obj & Other & Actor & Obj & Other & Actor & Obj & Actor & Obj & Other \\
\hline Women & 0 & 3 & 2 & 0 & 7 & 4 & 0 & 5 & 1 & 3 & 2 \\
\hline
\end{tabular}

Note. Obj = Object.

Table 14: Totals of women as actors and objects in the mixed corpus

\begin{tabular}{cccccc}
\hline & Lie (6) & \multicolumn{2}{c}{ Hate $(7)$} \\
\hline & Actor & Object & Other & Actor & Object \\
\hline Women & 3 & 0 & 3 & 0 & 7 \\
\hline
\end{tabular}

As these totals show, across all three corpora, women are more commonly the passive object that undergoes the action rather than the dynamic force behind the action. Of these collocates, women are only actors of the verbs think (one instance) and lie (three instances). Given this, I argue that tweeters do not discursively construct women in these corpora as frequently having agency. Additionally, when women have agency, it is most frequently associated with spreading false information. This result strongly implies that tweeters construct this social actor negatively. As further social actor analysis will show, this finding of the discursive construction of women in the three corpora recurs when I analyzed randomly sampled tweets for social actor representations.

\subsubsection{Role allocations for women}

The remaining subsections detail the results from analyzing the thirty-one downsized and randomly sampled tweets from the three corpora. Each of the following subsections investigates a different representation and the totals from the analysis of this representation. In the discussion chapter, I give my interpretation of these findings. 
Continuing to examine what roles tweeters allocated to women in the corpora, I analyzed whether women were activated (the dynamic force of the action) or passivated (the role undergoing the action) (Table 15). Under the category of passivation, this also included determining whether women were subjected social actors (treated as an object) or beneficialized ones (positively or negatively benefiting from the action). Across the corpora, passivation of women occurred four more times (twenty-eight) than activation (twenty-four). Of the categorizations of passivated social actors, it is always more common for women to be subjected social actors than beneficialized ones regardless of the corpus. In the \#KickVic corpus, the passivation of women is more frequent than activation. Contrastively, activation and passivation of the social actor women occurred an equal number of times. Lastly, the randomly sampled tweets from the mixed corpus contained four instances of women as activated social actors and three examples of women as passivated social actors. A comparison of these totals exists in Table 15.

Table 15: Totals of activated/passivated social actors who are women

\begin{tabular}{cccc}
\hline & Activation & \multicolumn{2}{c}{ Passivation } \\
\cline { 3 - 4 } & & Subjection & Beneficialization \\
\hline \#KickVic & 11 & 15 & 1 \\
\#IStandWithVic & 10 & 5 & 3 \\
Mixed & 4 & 3 & 0 \\
\hline Total across corpora & 25 & 23 & 4 \\
\hline
\end{tabular}

However, I not only wanted to know whether women were activated or passivated social actors in these thirty-one tweets but what actions were associated with these representations across the three corpora. Therefore, I compiled every dynamic force related to women from the tweets and categorized these results based on the corpora and whether the action came from women (Table 16) or was done to women (Table 17). 
Interestingly, the number of actions women activated, and the number of actions women underwent are very similar within each corpus. For example, in the \#KickVic corpus, women are the dynamic force behind fifteen actions, and they also undergo fifteen actions from the fifteen tweets I analyzed from this corpus. Likewise, I analyzed twelve tweets from the \#IStandWithVic corpus and again, women are the dynamic force behind thirteen actions, and they undergo eleven actions. Lastly, of the four tweets I analyzed from the mixed corpus, women are the dynamic force behind five actions, and they undergo four actions.

In my view, it is also important to examine what types of actions are associated with women in these allocated roles. Of the thirty-three actions identified in Table 16, eighteen of these actions construct the social actor women as liars and complainers, as those who forgive criminals, as irresponsible or persuadable, and who are malicious. The other fourteen actions include verbal processes (say, cry assault, come forward, report and call), mental processes (believe and think), material processes (count, do nothing wrong, deserve, and use) and an attribute (innocent). Across the corpora, negative constructions of women's actions occur in seven out of fifteen of \#KickVic's actions, eight out of thirteen of \#IStandWithVic's actions, and two out of five of the actions from the mixed corpus. Table 16 details which of these actions belong to each corpus. 
Table 16: Active forces of women across corpora

\begin{tabular}{|c|c|c|c|}
\hline & \#KickVic & \#IStandWithVic & Mixed \\
\hline Actions & $\begin{array}{c}\text { say, [to be] a liar, } \\
\text { forgive (a wife abuser), } \\
\text { say, [to be] more like } \\
\text { men, cry assault, lie, } \\
\text { come forward, say, } \\
\text { [don't] count, lie, make } \\
\text { up (sexual harassment), } \\
\text { [need to be] more } \\
\text { responsible, [to do] } \\
\text { nothing (wrong), report }\end{array}$ & $\begin{array}{l}\text { complain, [to be] } \\
\text { innocent, [to get] } \\
\text { sick (fed up), } \\
\text { deserve (fair } \\
\text { treatment), [not be] } \\
\text { easily led, believe, } \\
\text { ruin, [to be] awful, } \\
\text { lash out, [can be] as } \\
\text { bad (as bad men), } \\
\text { call, kick out, think }\end{array}$ & $\begin{array}{l}\text { [don't] lie, ruin } \\
\text { (someone's } \\
\text { life), say } \\
\text { (statistics), use } \\
\text { (statistics), } \\
\text { [don't] know } \\
\text { (other women) }\end{array}$ \\
\hline tal in corpus & 15 & 13 & 5 \\
\hline
\end{tabular}

Likewise, I examined the types of actions women undergo, whether they be subjected to the action or benefit from it. Across the three corpora, women undergo thirty actions and a few of these actions are repeated (abused, beaten, believed, and hated). Of these thirty actions, women endure physical, emotional, or mental harm from thirteen of them (e.g., abused, beaten, touched and kissed non-consensually, banned, harassed, and hated). Also, seven of these actions relate to women undergoing a form of support (believed, respected, and accepted) or protection (e.g., protected, defended, and prevented from being objectified). There are also two verbal processes (told and spoken for), five material processes (studied, sided with, tiptoed around, given, and involved), and one attribute (to be women). Across the corpora, nine out of fifteen of \#KickVic's actions construct women as subjected to harm, three out of eleven of \#IStandWithVic's actions construct women as subjected to harm, and one out of four of the tweets from the mixed corpus construct women as subjected to harm. Table 17 details which of these actions belong to each corpus. 
Table 17: Active forces women undergo across corpora

\begin{tabular}{|c|c|c|c|}
\hline & \#KickVic & \#IStandWithVic & Mixed \\
\hline Actions & $\begin{array}{l}\text { abused, beaten, abused, } \\
\text { gotten (worse for), } \\
\text { believed, protected, } \\
\text { touched (non- } \\
\text { consensually), abused, } \\
\text { defended, prevented } \\
\text { (from being objectified), } \\
\text { told, studied, banned, } \\
\text { kissed (non- } \\
\text { consensually), harassed }\end{array}$ & $\begin{array}{l}\text { beaten, [to be] a } \\
\text { woman, sided } \\
\text { (with), spoken } \\
\text { (for), hated, tiptoe } \\
\text { (around), hated, } \\
\text { respected, [to be] a } \\
\text { part of a man's sex } \\
\text { life, [should be] } \\
\text { accepted, [should } \\
\text { be] believed }\end{array}$ & $\begin{array}{l}\text { given (power), } \\
\text { abused, [to be] } \\
\text { women, } \\
\text { involved (in a } \\
\text { conspiracy) }\end{array}$ \\
\hline al in corpus & 15 & 11 & 4 \\
\hline
\end{tabular}

\subsubsection{Characterization of women}

One finding that the social actor analysis provided is that women collectively are not the only women in these tweets. Instead, individual women are also a part of this random sample. Because of the previous finding where the plural form of women is more frequent than the singular form of woman, I decided to investigate this further in terms of individualization and assimilation. The former representation is broken down further into collectivization, where people are denoted as a group of unspecified size, or aggregation, which "is realized by the presence of definite or indefinite quantifiers" (van Leeuwen, 2008, p. 38). As Table 18 shows, the assimilation of women is more common in the down-sampled tweets from each corpus (Examples 10 and 11) than individualization is (Example 12). Example 10 represents collectivization, example 11 shows aggregation, and example 12 illustrates individualization and collectivization.

Example 10: \#IStandWithVic

Men are guilty until they are proven innocent and women are innocent until they are proven guilty. FUNimation have proven it this time. 
Example 11: Dozens of women and girls: "Vic sexually abused me"

Con staffers and industry insiders: "He has been a creepy asshole for years and it needs to stop"

Random people who probably only meet Vic briefly at a convention, if at all: "YOU'RE LIARS, HE'S A GOOD GUY!"

\#KickVic

Example 12: I am a woman myself and I am getting sick of everyone siding with them until they are proven guilty. Men deserve the fair treatment just as women do. \#IStandWithVic \#IStandWithIndividual_F [hashtags removed] [hyperlink removed]

In the \#KickVic tweets, assimilation occurs fourteen times, whereas individualization occurs seven times. Likewise, I identified twelve examples of assimilation in the \#IStandWithVic tweets and three examples of individualization. Lastly, there are five instances of assimilation in the mixed corpus tweets and only one occurrence of individualization. Although I identified examples of women being aggregated in the tweets from each corpus, it is more common in the \#KickVic tweets and the \#IStandWithVic tweets to refer to women as a collective. A comparison of these totals exists in Table 18.

Table 18: Totals of individualized/assimilated social actors who are women

\begin{tabular}{cccc}
\hline & Individualization & \multicolumn{2}{c}{ Assimilation } \\
\cline { 3 - 4 } & & Collectivization & Aggregation \\
\hline \#KickVic & 7 & 9 & 5 \\
\#IStandWithVic & 3 & 9 & 3 \\
Mixed & 1 & 2 & 3 \\
\hline Total across corpora & 11 & 20 & 11 \\
\hline
\end{tabular}


Related to individualization and assimilation are the social actor representations of categorization and nomination (Table 19). Much like assimilation, categorization involves referring to social actors "in terms of identities and functions they share with others" (van Leeuwen, 2008, p. 40). Contrastively, nomination occurs when social actors are represented by who they are, including their name. For this analysis, I added the category username for when nominated social actors are represented by their online identity. Like the previous findings, categorization is more common than nomination strategies. In the \#KickVic tweets, categorization occurs fifteen times, whereas nomination occurs seven times. Likewise, I identified twelve examples of categorization in the \#IStandWithVic tweets and two examples of nomination. Lastly, there are five instances of assimilation in the mixed corpus tweets and only one occurrence of nomination. Of the nomination representations, it is more common to refer to individual women informally (with their given name) or with their username (each representation occurs three times). Between the previous and current findings, in this down-sample collection of tweets, it is more common to refer to women as a collective group than as unique individuals. While individualization and nomination are not absent from these tweets, these results replicate what I found with the word frequencies across the corpora. On the large- and small-scale level, women are more frequently discursively constructed as a collective in these tweets related to this protest and counter-protest. I will explore the implication of this finding alongside others in the following chapter. Table 19 contains the totals of nomination and categorization across the three corpora. 
Table 19: Totals of categorized/nominated social actors who are women

\begin{tabular}{cccccc}
\hline & Categorization & \multicolumn{4}{c}{ Nomination } \\
\cline { 3 - 6 } & & Semi-formal & Informal & Username & Other \\
\hline \#KickVic & 15 & 2 & 1 & 3 & 1 \\
\#IStandWithVic & 12 & 0 & 1 & 0 & 1 \\
Mixed & 5 & 0 & 1 & 0 & 0 \\
\hline Total across & 32 & 2 & 3 & 3 & 2 \\
corpora & & & & & \\
\hline
\end{tabular}

\subsubsection{Us versus Them}

Of all the recurring patterns from this sample of tweets, the final one I investigated across the three corpora is the discursive construction of Us versus Them from van Dijk (1998). In the thirty-one tweets analyzed, I discovered two categories of Us versus Them: Us men versus Them women (type one) and Us women versus Them women (type two) (Table 20). Example 6 (Section 4.4.1) illustrates Us men versus Them women, and example 13 represents the category of Us women versus Them women.

Example 13: And just as many come forward saying "This was consensual, Stop using my pictures". But those women do not count I guess. And you want to talk about a load of crap? Lets talk about Individual_W lying to the public about a fake SWAT. You \#kickvic guys have 0 credibility.

For the \#KickVic tweets, the first type of squaring occurs three times, and the second type occurs twice. Contrastively, I identified two instances of type one and three occurrences of type two in the \#IStandWithVic tweets. Lastly, there is only one example of Us men versus Them women ideological squaring in the tweets from the mixed corpus. This first type of squaring is more frequent in all the tweets but only by one example. Usually, tweeters construct the divisions between these groups of social actors are constructed 
through actions of attributes. An example of the first type of ideological squaring from the \#KickVic tweets involves men, who are the victims of digital activism, positioned against women who 'cry wolf' and need to be more responsible with their power. For the second type of squaring, examples from the \#IStandWithVic tweets include women who do not complain versus women who do, women who are logical versus illogical women, and women who are awful because they use digital activism as means to enact social change versus women who are 'real' and fight back when they are harassed. Altogether, there are eleven examples of these two types of ideological squaring across the three corpora (Table 20).

Table 20: Totals of Us versus Them

\begin{tabular}{ccc}
\hline & Us men vs Them women & Us women vs Them women \\
\hline \#KickVic & 1 & 2 \\
\#IStandWithVic & 2 & 3 \\
Mixed & 1 & 0 \\
\hline Total across corpora & 4 & 5 \\
\hline
\end{tabular}

\subsection{Summary}

With several types of analysis from various approaches completed, it is clear that many procedures corroborate the same findings. Firstly, thanks to the general layout of the corpora that the word frequency lists provided, I observe that tweeters reference men more frequently than women. Additionally, I learn that it is more common for man singularly to occur in the corpora than men collectively. Contrastively, women collectively occurs more often in the three corpora than woman singularly. From the analysis of semantic themes and keywords, we see that while the corpora share many of the semantic themes identified, the keywords associated with these semantic themes vary depending on the corpus. Also, I argue from the similarity and difference findings that, 
against a general reference corpus of Englishes, the \#IStandWithVic corpus and the mixed corpus are the most like each other, and the \#IStandWithVic corpus is the most unique of the three corpora. However, according to the direct corpus comparison, the \#IStandWithVic corpus and the mixed corpus are the most similar, and the \#KickVic corpus is the most unique of the three. Lastly, the in-depth analysis of the social actor women showed that, in the down-sampled collection of tweets, tweeters do not discursively construct women with much agency, and when they do have agency, it is over actions that cause harm to others. As insinuated, I further discuss my interpretation of these findings in the next chapter alongside their implications for the social change of this context, feminist critical discourse analysis, and my research questions. 


\section{Chapter 5: Discussion}

In this chapter, I initiate a dialogue between the research questions and my findings by summarizing the quantitative and qualitative analyses. The purpose of this chapter is to tie together the theoretical concepts and the previous studies reviewed earlier in this thesis (Chapter 2) to what I have discovered. According to Chapter 1, this project aims to understand how this protest and counter-protest critique or reinforce the power imbalances embedded into our societies to enact or prevent social change. In this dialogue, I also provide my interpretation of the results where my theoretical framework helps me to understand my findings. This project encompasses a niche and, to my knowledge, the theoretical considerations I used have yet to be applied to the context of a protest and counter-protest where tweeters used hashtags in the service of digital activism.

As a reminder, a few parts comprise this niche that my project addresses. Firstly, scholars have studied the hashtags of protests and counter-protests for various purposes (Barker-Plummer \& Barker-Plummer, 2017; Clark, 2016; D’Ambrosio, 2019; Gallagher et al., 2018; Palomino-Manjón, 2020; Zhu, 2016), but as I understand, no one has researched the \#KickVic and \#IStandWithVic hashtags. Additionally, only one study that examined hashtags used in the service of digital activism where there is a protest and counter-protest hashtag created two corpora and addressed those tweets that contained both hashtags (Gallagher et al., 2018). Furthermore, a few studies draw on Zappavigna's (2011) concept of ambient affiliation (D’Ambrosio, 2019; Palomino-Manjón, 2020; Zhu, 2016), but none also consider Gee's (2005) concept of affinity spaces for digital activism. To my present knowledge, no study exists that uses the concepts of affinity spaces and 
ambient affiliation to theorize hashtags in the service of digital activism on Twitter, where CL, CDS, and FCDA comprise the theoretical and methodological considerations, and that constructs two or more corpora around protest and counter-protest hashtags. Taken together, the connections that I will establish in this chapter contribute to an original and salient niche in this field of research.

\subsection{Restatement of research questions}

One aim of this study was to investigate how the discursive constructions of this instance of digital activism patterned in terms of the similarities and differences that arose from the tweets that used \#KickVic, the tweets that used \#IStandWithVic, and the tweets that used both hashtags. Additionally, I investigated how Twitter users who posted tweets with these protest and counter-protest hashtags discursively constructed the social actor of women because of the importance and relevance they have regarding the sexual harassment allegations of this context. Considering the critical perspective this project must address due to how the \#KickVic hashtag critiqued a social issue in society and raised awareness for it through activism, this discussion also examines the extent to which Twitter users subvert or perpetuate dominant patriarchal ideologies. For these purposes, the results of my analysis answer the following questions:

1. How do the similarities and differences of keywords and semantic themes discursively construct the protest and counter-protest?

2. How do Twitter users discursively construct women in this protest and counterprotest? 
3. To what extent do these findings subvert or perpetuate (i) dominant ideologies related to sexual harassment and (ii) digital activism hashtags to address this social issue?

The remainder of this chapter addresses the answers to these questions and interprets their implications through the theoretical considerations of this project. I provide a summary of the discussion and these implications at the end of the chapter.

\subsection{Question 1}

Question 1 of this thesis asks: How do the similarities and differences of keywords and semantic themes discursively construct the protest and counter-protest? The results of my similarities and differences analysis provide supporting evidence that each corpus talks about the same issues as the others (via recurring semantic themes) but that they each construct these issues differently at the lexical level (via different and unique keywords). Additionally, the recurring semantic themes and unique keywords generated from the comparison against the AmE06/BE06 strongly imply what issues and lexical content are unique for my corpora, and therefore this protest and counter-protest. Firstly, the issues that all three corpora address are Mignogna, what counts as valid evidence, spaces where sexual harassment occurs or where digital activism happens, the relevant groups, people, and specified individuals of this context, the attributes of social actors in this situation, other websites on the Internet, who comprise a collective, and the actions related to the effort of reporting sexual harassment. An issue that Twitter users talk about in the \#KickVic corpus and the mixed corpus is the present protest. For these two corpora, the digital protest is also a thing, as examples 14-17 exemplify: 
Example 14: without consent and rallying up his fans against accusers. Did you hear about the whole \#KickVic thing?

Example 15: Yeahhh tru ppl are saying its because of the whole \#KickVic thing but $i$ think it was to speed up localization

Example 16: What's this about Toei being and at funimation over the \#KickVic \#IStandWithVic thing@individual_X I'm randomly getting these things recommended to me and it's annoying

Example 17: Can someone explain the whole \#IStandWithVic \#KickVic thing. Also why would RT fire him on a claim?

Secondly, the \#KickVic corpus also discursively constructs the social issue — and uses the hashtag to index this - as a movement (exemplified by its position as a unique keyword to this corpus). This pattern is consistent with the previous literature as D'Ambrosio (2019) discusses the lexical item movement in a study concerning a \#BlackLivesMatter corpus. The item movement appears on the frequency list for this corpus and D'Ambrosio (2019) suggests this signifies that "the corpus revolves around the protest against police brutality and racial inequality itself' (p. 18). Because movement is a unique keyword in the \#KickVic corpus, I believe that part of this corpus revolves around the protest of sexual harassment and how patriarchal ideologies work to minimize its importance as an issue within society. Contrary to this, the mixed corpus does not discursively construct the social issue in the same way. In the mixed corpus, tweeters use the hashtags \#KickVic and \#IStandWithVic together to represent this situation so the hashtags seem like they index two sides of the same coin. This idea is further supported by the findings from the differences section in Chapter 4 as I believe that the unique keywords versus and sides 
discursively constructed the hashtags of the mixed corpus as battling for dominance. While this might be true, it seems that tweeters use the hashtags together as a point of reference to ask for further clarification concerning the protest and counter-protest (Examples 16-17).

Returning to the keyword thing, the finding that both the \#KickVic corpus and the mixed corpus represent this social issue as a thing supports the idea that these tweeters downplay the significance of the protest and counter-protest. From this finding, I assume that this means some people posted tweets with the \#KickVic hashtag that either purposefully or unknowingly minimized the importance of this hashtag by discursively constructing it as an unspecified object (Examples 14-15). The theoretical notion of ideology from CDS and FCDA helps me understand this finding, as patterns of meaning shape discourse that become embedded with ideological structure. Taken together, I argue that while the protest is a commonly referred to issue in the \#KickVic corpus and the mixed corpus, how certain Twitter users talk about this protest subverts its overall importance by referring to it with a label that reduces it from a legitimate social issue to a generic non-entity.

Additionally, the keyword and semantic theme harassment is a significant issue and lexical item that users talk about in these two corpora:

Example 18: \#Convention_Y $Y^{5}$ is now paying to advertise with YouTube streamers who regularly encourage their fans to harass Vic's victims.

\footnotetext{
${ }^{5}$ This is a pseudonym. I have replaced the name of the convention with an alphabetical symbol.
} 
Any money spent at \#Convention_Y2019 is supporting this ongoing abuse. It's time to take a stand against sexual harassment. \#BoycottConvention_Y \#KickVic [hyperlink removed]

Example 19: Harassing people? And what does \#KickVic hashtag do for Vic? Don't you call that harassment?

Example 20: @KamehaCon I hope you do not give in to the threats and harassment from \#kickvic. \#IStandWithVic stands with you and the decision you made and will support you in any way we can.

Example 21: all I did was send 을 emojis to her recent tweet, and then this happens, bravo well played, I guess it is harassment to laugh now \#IStandWithVic \#KickVic \#vickickback [hyperlink removed]

Examples 18-21 highlight that the tweets of these two corpora acknowledge (sexual) harassment allegations. Furthermore, these examples provide evidence that users talk about what constitutes harassment and who is the agent of harassment in this protest and counter-protest. For example, in the \#KickVic corpus, Twitter users discuss how Mignogna sexually harassed others (Example 18) and how the existence of the \#KickVic hashtag is harassment towards Mignogna (Example 19). This finding implies that the discursive construction of this semantic theme and keyword (harassment) is not consistent throughout all the tweets of this corpus, even though they share a hashtag. Rather than the \#KickVic hashtag only consisting of tweets that support the reactionary call for Mignogna to be held accountable for actions, tweeters also use this hashtag to reference the protest or the people who are a part of the protest (Examples 19-21). One 
interpretation of this finding is that hashtags used in the service of digital activism are fluid (intertextuality and dialogism). In my view, example 19 also provides evidence of the hijacking strategy that scholars have previously identified because the tweeter invokes the \#KickVic hashtag to discursively construct those of the protest as the harassers (Barker-Plummer \& Barker-Plummer, 2017; Gallagher et al., 2018).

In this case study, my findings highlight that hijacking occurs when those of the politically opposite stance (\#IStandWithVic) invoke \#KickVic to discursively construct the protest as harassment and therefore inject the hashtag with their ideological beliefs that the people of this stance are the harassers. Stance is a term discussed in the literature that studies how tweeters use hashtags in the service of digital activism (D'Ambrosio, 2019; Gallagher et al., 2018; Palomino-Manjón, 2020) and means how users position themselves concerning an issue. As well, Zappavigna (2011) notes that the evaluation observed in hashtags as indexers of digital activism allows Twitter users "to build power and solidarity by adopting stances and referring to other texts" (p. 794). For this protest and counter-protest, the social issue that users position themselves with is the sexual harassment allegations against Mignogna. These allegations are also the content that generates the affinity space of the protest and counter-protest. In this protest and counterprotest, tweeters ambiently affiliate around this content through their ideological beliefs and values that they implicitly embed in their tweets. One interpretation of this finding is that \#IStandWithVic perpetuates their discursive construction of harassment by hijacking the \#KickVic hashtag. Furthermore, invoking this construction alongside \#KickVic subverts the digital protest's importance and depicts the protest negatively. In my view, equating the purpose of \#KickVic as equal or worse than Mignogna's acts of sexual 
harassment discursively constructs the \#KickVic hashtag as the perpetrator and Mignogna as the victim in this context. Taken together, these findings show that while the \#KickVic corpus and the mixed corpus share more semantic themes (not keywords) than the \#IStandWithVic corpus (when compared against the AmE06/BE06, Section 4.3.1), the discursive constructions of the \#KickVic hashtag are uniform (Examples 18-21). Overall, the \#KickVic corpus is not consistent in terms of the discursive constructions that exist within it as Twitter users of the counter-protest hijack it to perpetuate their political stance.

Another finding of my analysis is that I did not identify the words thing or harassment as top-ranked keywords in the \#IStandWithVic corpus when I compared it against the AmE06/BE06. Additionally, these keywords are not top-ranked in the \#IStandWithVic corpus, and I did not identify any lexical items (keywords) related to the issues (semantic themes) of the protest or harassment when I compared this corpus against the AmE06/BE06. These results strongly imply that tweeters who add this hashtag to their tweets discuss other, more top-ranked, issues. These include semantic themes I have previously observed in the other corpora, including the discussion of Mignogna as a person, what counts as evidence, other (affinity) spaces and individuals involved with this protest and counter-protest, and various actions of social actors. Because this study focused on lexical items that are present in the corpora rather than those that are absent, I do not want to speculate too much. However, one interpretation of these findings is that the lack of shared or unique words of these semantic themes, when compared against the AmE06/BE06, indicates that Twitter users discursively construct the social issue indexed by the hashtag with other issues. 
To understand how users discursively construct the issues of the protest in the \#IStandWithVic corpus, I address the findings from the direct corpus comparison where I categorized the lexical item petition as part of the semantic theme for the protest:

Example 22: We the Fans would like Vic Mignogna back as a guest for 2020 Sign the Petition! \#IStandWithVic \#ISWV [hashtags removed] [hyperlink removed]

Example 23: Speaking of @RoosterTeeth and @FUNimation PR disasters, the Vic \#RWBY petition is nearly at 5k. \#IStandWithVic [hyperlink removed]

Firstly, these two examples of this word highlight that Twitter users circulate many petitions of various causes alongside the hashtag \#IStandWithVic. Furthermore, only five of the instances of this word are not accompanied by an external link to a petition website. These results imply that tweeters discursively construct petition as a protest for change because these petitions ask for Mignogna to be reinvited as a guest for conventions, for him to be rehired, and for Toei (a Japanese publishing company) to provide aid for Mignogna. While these petitions discursively construct \#IStandWithVic as a group of supporters who want Mignogna to return and be reaccepted by the industry, other petitions in this corpus discursively construct another ideological position. In this corpus, some petitions demand the boycott of Funimation and Rooster Teeth and for the dismissal from conventions and the industry of two of Mignogna's colleagues who shared their sexual harassment experiences concerning him. For the tweeters who use \#IStandWithVic, the protest is not a movement advocating for accountability (\#KickVic) or a thing that is an event (\#KickVic and mixed tweets) but an opportunity to express their support for Mignogna. In my view, tweeters share these petitions to dismiss the work of 
the protest and harm those individuals that have spoken up concerning the social issue of sexual harassment. Lastly, these petitions discursively construct \#IStandWithVic as a helpful force because it advocates for Mignogna's justice while also subverting the significance of \#KickVic by constructing it as a damaging collective. This finding may be explained by the idea of van Dijk's (1998) ideological square as tweeters express positive information about \#IStandWithVic positive and suppress positive information about \#KickVic. Taken together, the two different types of petitions benefit each other in this discursive construction because by categorizing and collectivizing \#KickVic as the offenders, the petitions help Mignogna rebuild his reputation. Overall, this construction of social change as victimization for the politically opposite stance not only erases the significance of the digital protest but instead characterizes it as an issue that requires change. This discursive construction of victimization, and therefore the delegitimization of \#KickVic, is also observed in D'Ambrosio (2019) where tweeters use “white victimhood" to counter-resist the \#BlackLivesMatter protest (p. 24). Through victimization and representing the protest as a petition, tweets with the \#IStandWithVic hashtag centre this counter-protest affinity space as about justice for Mignogna, therefore legitimizing this stance and "[distorting the protests'] original meaning” (D'Ambrosio, 2019, p. 24).

As this discussion illustrates, the three corpora talk about the same issues but use different lexical items that discursively construct the protest and counter-protest differently. Furthermore, even though two corpora use many of the same lexical items to refer to issues, this does not mean that users construct the issue with uniformity throughout the protest. In this case study, my findings highlight that while the mixed 
corpus is more similar to the \#KickVic corpus for the issues of protest and harassment, the discursive construction of this issue in the mixed corpus shares more similarities with the discursive construction of the same issue in the \#IStandWithVic corpus. Additionally, there exists this same politically opposite discursive construction in the \#KickVic corpus which exemplifies the fluidity of constructions in this protest and counter-protest. Overall, the similarities and differences of these three corpora demonstrate that the hashtag is not a marker for how the protest and counter-protest will be discursively constructed in the tweet.

In my view, the most compelling explanation for the present set of findings is that of the theoretical concept of affinity spaces. The hashtags, which are both portals into the affinity spaces, are used to refer to the affinity spaces and serve as permeable boundaries to the affinity spaces, exist on a continuum. At one end of the continuum, there exists \#KickVic, which represents the protest and those people advocating for social change. On the other end of the continuum exists \#IStandWithVic, where Twitter users of the politically opposite stance to \#KickVic advocate for Mignogna's justice and the maintenance of the social norm. In the middle, some tweets use both hashtags, and the discursive constructions of these tweets can mirror \#KickVic or \#IStandWithVic. Though there are tweeters who are on the protest side and those who are on the counter-protest side, these two affinity spaces are inherently connected because of how tweeters created the hashtags. \#KickVic is a response to the circulating sexual harassment allegations. Similarly, \#IStandWithVic is a response to \#KickVic's purpose of creating awareness about the social issue. Because the protest and the counter-protest discuss the same issues (semantic themes), it is not easy to determine where one affinity space ends and the next 
one begins, especially considering that some tweets include both hashtags. While I discovered various discursive constructions associated with each corpus, it is not possible to say, for example, that only discursive constructions related to the protest exist within the \#KickVic corpus. For reasons due to these hashtags' functions (portals, reference points, and boundaries), there are no rules in place that stipulate who can and cannot use a hashtag, and the connectivity of these hashtags. The notion of this affinity continuum returns in the discussion of Sections 5.3 and 5.4.

Furthermore, I believe Gee's (2005) concept of affinity spaces is a useful theoretical concept for understanding two of the hashtags of this protest and counterprotest because we see that tweeters built this affinity continuum by socially affiliating around the sexual harassment allegations attributed to Mignogna. The protest and counter-protest sides each have similar endeavours which are exemplified by the lexical items that construct each hashtag. Everyone, from fans, alleged victims, Mignogna's supporters, voice actors, and companies share the space of this continuum. The portals (hashtags) are strong generators, as they are both content of the continuum and access points to the continuum. Furthermore, there are different ways to participate within this continuum, as signified by the many ways tweeters use the hashtags attributed to this protest and counter-protest. Taken together, the findings for this research question exemplify the fluidity in discursive constructions as they relate to the affinity continuum of this protest and counter-protest and provide further evidence that a hashtag cannot by itself indicate where along the continuum the tweet belongs. 


\subsection{Question 2}

Question 2 of this thesis asks: How do Twitter users discursively construct women in this protest and counter-protest? The analysis of nomination and predication patterns for social actor representations illustrates that across the three corpora Twitter users construct women similarly and differently from each other. Firstly, these findings highlight that Twitter users more frequently discursively construct women as a collective in both the protest and the counter-protest rather than referencing specific individuals who are women. One interpretation of these findings is that the various purposes of this collectivization have different ideological implications between the protest and counterprotest and within one side of this protest. These results imply that certain Twitter users collectivize women to show their support for all women and that they used this representation to shame all women or shame particular collectives of women. Taken together with the lack of a collective women's agency for actions other than those that cause harm to others (Table 16), Twitter users do not positively discursively construct women in this protest and counter-protest. The present results are consistent with previous studies that show other digital activism hashtags also perpetuate oppressive ideologies towards women within the original protest hashtag (Palomino-Manjón, 2020; Zhu, 2016) and in opposing hashtags (Barker-Plummer \& Barker-Plummer, 2017). This idea is further supported by my observation that Twitter users perpetuate the power asymmetries of gender ideologies in both the protest hashtag (\#KickVic) and the opposing hashtag (\#IStandWithVic). Furthermore, this perpetuation also occurs when tweeters use the hashtags together. 
In my view, the most compelling explanation for the current set of findings is the affinity continuum I coined in Section 5.2. On \#KickVic's end of the ideological continuum, Twitter users support women, believe in their allegations, and know they are not lying because these users know other women who have been sexually harassed. Through this, the protest acknowledges that sexual harassment is a societal issue, and these tweeters create awareness to address this gendered ideological structure. Additionally, those of \#KickVic's political stance assimilate all women to address how sexual harassment is a worldwide issue and to emphasize that those women who have been sexually harassed are not isolated instances. Altogether, tweeters of \#KickVic's position discursively construct the sexual harassment allegations of these women as a critical issue and through the ideologies embedded in their tweets, they subvert oppressive patriarchal ideologies.

At \#IStandWithVic's side of this social issue, Twitter users discursively construct women, or the collective of women, differently. For example, users of the counter-protest distance themselves from those of \#KickVic by saying that those of the protest should not speak for all women. This idea is supported by the results from the representation of Us \#IStandWithVic women versus Them \#KickVic women where Us emphasizes Them as committing a wrongful deed (challenging the social issue). Additionally, Twitter users of this position discursively construct women, and sometimes specifically those women of \#KickVic, as the agents of malicious actions (ruin, lash out, kick out). Lastly, tweeters of \#IStandWithVic use assimilation to refer to the social group of women as a whole and discursively construct that whole as a problem for the social norm. Taken together, Twitter users of \#IStandWithVic's political stance discursively construct the women of 
\#KickVic as the issue that society must be wary of, thereby perpetuating oppressive patriarchal ideologies by reinforcing the need for them in society through ideological squaring. This reinforcement is explained by the theoretical considerations of CDS and FCDA as they discuss how ideology and power are intertwined because those individuals that uphold the stance of the social norm use discursive constructions in these affinity spaces to reinforce ideologies. D'Ambrosio (2019) also discusses this idea of strengthening oppressive ideologies within the context of race and "the institutionalized force of police" in the study of \#AllLivesMatter and \#BlueLivesMatter in relation to \#BlackLivesMatter (p. 7). Taken together, reinforcing ideologies that lead "to the emergence of counter-[protests] aimed at delegitimizing the demands put forward by" the original protest is a recurring finding (D'Ambrosio, 2019, p. 6).

Finally, I obtained evidence that tweets containing both hashtags fall belong to the middle of the affinity continuum because the posts discursively construct women in ways that mimic the continuum's ends. This result strongly implies that subversion and perpetuation of patriarchal ideologies exist in tweets that use both \#KickVic and \#IStandWithVic. In the four tweets analyzed with both hashtags, three tweeters used one hashtag to express their political stance and the other hashtag to refer to the other end of the continuum (Examples 6, 24-25).

Example 24: It looks like $90 \%$ of the \#IStandWithVic users in this thread are women. So much for the sexist "all Vic fans are 14 year old alt-right white boys" remarks/narrative. \#kickvic must be getting desperate.

Example 25: Re: \#IStandWithVic

You all really out here arguing that it's more likely there's a character 
assassination conspiracy spanning 15 years, hundreds of women who don't know one another, con staffers and his own fucking co-workers than one person lying.

\#KickVic

Additionally, the political stances of the hashtags at the continuum's ends also act as access points to the discussion, allowing Twitter users of \#IStandWithVic's politically opposite position to hijack the \#KickVic hashtag (Section 4.4.1, example 6; example 24). In my view, I believe this affinity continuum and its fluidity allow these tweeters to inject their ideological beliefs and perpetuate the oppressive patriarchal ideology anywhere along the affinity continuum of this protest and counter-protest.

The findings of my analyzes show that, of the tweets I collected, women are not a central issue despite their significant relevance to the social issue that tweeters used to create this affinity space. With this evidence, I argue that tweeters of this continuum hardly discuss the women's collective. Instead, the discursive constructions focus on other issues, like other groups and individuals involved, the actions and attributes of these social actors, and the evidence of this protest and counter-protest. Additionally, the findings of the discursive constructions of women highlight that Twitter users construct this social actor positively (from tweeters who agree with \#KickVic) and negatively (from tweeters who agree with \#IStandWithVic). The theoretical notions of CDS/FCDA exemplify why these discursive constructions exist within this affinity continuum. As a reminder, tweeters created the continuum that I refer to here with the content of the sexual harassment allegations attributed to Mignogna. On this continuum, there exists the overarching ideological structure discussed by Lazar (2007), where society divides 
people into two classes (men and women) based on domination (by men) and subordination (of women). Under men's authority, women are disadvantaged, excluded, and disempowered as a social group, which manifests, for example, as challenges when reporting sexual harassment (Section 1.3). Even though women are a central social actor of this context, as they are the recipients of this alleged sexual harassment, they too are dominated within the corpora by other issues.

I further support this idea with the finding that tweeters negatively constructed and emphasized the women's collective many times along this continuum through various discursive constructions. The results of the collocate, role allocation, characterization, and ideological squaring analysis provide evidence for these negative constructions of women. For example, ideological squaring (van Dijk, 1998), a form of ideological reproduction, involves the presence or absence of certain semantic representations as they relate to groups involved. As such, a Twitter user may suppress the positive semantic representations of one group (Them) to express the positive representations of another (Us). At the same time, the tweeter emphasizes the negative semantic representations of the outgroup (Them) and suppresses the negative representations of the ingroup (Us). This sequence of moves allows the tweeter to highlight the positive qualities of the ingroup and the negative qualities of the outgroup. Additionally, these moves contribute to ideologies about social groups and perpetuate the ideologies of those groups that are positively represented and expressed. In my case study, tweeters perpetuate oppressive gender ideologies by positioning all men against all women and by positioning the women of \#IStandWithVic against those of \#KickVic. While certain tweeters discursively construct women through lexical choices and 
discourse strategies that empower this social actor, the above evidence highlights that people continue to disempower women by perpetuating this dominant ideology.

Firstly, these findings and interpretations produce a feeling of disappointment as the \#KickVic hashtag is an instance of digital activism that began thanks to victims and survivors of sexual harassment who wanted Mignogna to be held accountable for his actions. The efforts of alleged sexual harassment survivors and their supporters for this protest created tangible change in the voice acting industry and anime convention scene in the form of making those spaces safer for everyone involved. Despite this, the tweets of my corpora illustrate that Twitter users continuously discursively construct women as a malicious group whose efforts (for social change) need to be fought against to restore a patriarchal social order. For the tweeters of this stance, this involves allowing Mignogna to return to these spaces—-which they argue he was wrongfully dismissed from despite Funimation conducting their investigation into allegations (Loveridge, 2019c) —and jeopardizing the safety of vulnerable individuals (women). However, restoration of social order for the counter-protest also includes punishing women who used their agency irresponsibly to enact this social change. Furthermore, the counter-protest hashtag (\#IStandWithVic) holds these views and so do those tweeters who hijack \#KickVic to spread this message. Taken together with the findings of the first research question, I observe that within this affinity continuum, it is complex to separate the tweets of this protest and counter-protest.

In my view, these findings are not surprising because previous literature has found similar discourses in another hashtag used in the service of digital activism (Palomino-Manjón, 2020). Within the space of \#KickVic and \#IStandWithVic, the results 
from analyzing the social actor women demonstrate that individuals of the politically opposite stance reproduce power asymmetries (Lazar, 2017) through the discursive construction of women as the reason for why the social norm was upended. My findings highlight the identification of empowering discourses for the collective of women in the \#KickVic corpus and the mixed corpus (Section 4.4.3). Additionally, these findings also contain evidence that the reproduction of these power asymmetries occurs much more frequently in the down-sampled tweets I analyzed (Table 16; Examples 6, 10, 12-13). Overall, this abundant frequency of gendered power asymmetries results in the overwhelming discursive construction of the women's collective as to blame for Mignogna's suffering in both the protest and counter-protest hashtag. Though this discourse of male victimization and hate speech (Palomino-Manjón, 2020) did not inhibit the critique of the social issue of sexual harassment and the resulting awareness from this protest's advocacy, this discursive construction of women reinforces that these gendered power asymmetries continue to be held by those people who wish to uphold the status quo.

\subsection{Question 3}

Question 3 of this thesis asks: To what extent do these findings subvert or perpetuate (i) dominant ideologies related to sexual harassment and (ii) digital activism hashtags to address this social issue? The purpose of this study was to gain a better understanding of the similarities and differences through the discursive constructions of keywords and the social actor women that tweeters used in the affinity continuum attributed to the \#KickVic protest and the \#IStandWithVic counter-protest. Furthermore, I divided the tweets according to hashtags to examine if there was uniformity in the 
discursive constructions for a corpus constructed with, for example, the protest hashtag. The importance of my theoretical considerations, which involve affinity spaces, digital activism, CDS, and FCDA, is that these theories allow me to understand why I did not discover uniformity of discursive constructions with my corpora that represent this protest and counter-protest.

My findings highlight that the hashtags attributed to this affinity space constitute a continuum of political stances (D'Ambrosio, 2019; Gallagher et al., 2018; PalominoManjón, 2020). Reflecting on Section 5.2's discussion, tweeters created this affinity continuum using the content of the sexual harassment allegations attributed to Mignogna. On this continuum, where part of the discussion concerns power asymmetries and gender ideologies related to women, the hashtags \#KickVic and \#IStandWithVic indicate the permeable boundaries of this affinity continuum. I believe these boundaries are permeable because they overlap with other hashtags that tweeters use in the service of digital activism to raise awareness for similar social issues like \#MeToo, \#WhyIDidntReport, \#WhyIStayed and \#WhyILeft. However, as Gee (2005) notes, one aspect of affinity spaces that I argue contributes to this lack of uniformity in my corpora is that there are no "bosses" in an affinity space (p. 228). In my case study, leaders of the \#KickVic and \#IStandWithVic continuum "cannot order people around" and create rules that say who can and cannot use the hashtags in various tweets for various reasons ( $\mathrm{p}$. 228). This idea, coupled with the fact that Twitter users created the hashtags as responses to different content, means that I can account for the fluidity of these hashtags despite the surface opposition they represent. On this affinity continuum of \#KickVic and \#IStandWithVic, tweeters can affiliate around the content that generates the continuum 
however they wish because there are no strict rules regarding interaction within this space. For this reason, I found perpetuation of power asymmetries related to gender ideologies of men and women across all three corpora.

This idea is further supported by the finding that the \#KickVic affinity space is not purely a space of protest. Instead, \#KickVic allows Twitter users that share its political stance to advocate for social change and permits those tweeters who are of the opposite political stance to discursively construct this protest as harmful. Additionally, my findings strongly imply that when tweeters use the counter-protest hashtag (\#IStandWithVic) without the other (\#KickVic) that it only acts as a space for those users who share its political stance. Lastly, the mixed corpus and down-sampled analysis of women showed that tweeters of \#KickVic's position use the \#IStandWithVic hashtag, but in these cases, they also hashtag the tweet with \#KickVic. Whereas past research has found that the protest hijacks the counter-protest hashtag (Gallagher et al., 2018), this thesis observes a different usage of stance. In the previous literature, they found that "advocates of \#BlackLivesMatter often use \#AllLivesMatter to directly interrogate the stance of 'All Lives Matter' and the worldview implied by that phrase" (p. 18). However, I discover the opposite in my study as tweeters of \#IStandWithVic's political position use \#KickVic to interrogate the stance of the protest and delegitimize its digital activism (Examples 4, 13, 18, 19, 29). Considering my findings, this continuum may be explained in the following terms. First, tweets hashtagged with \#KickVic can share the protest's political stance or oppose it. Secondly, tweets hashtagged with \#IStandWithVic can share the counter-protest's political stance and as my findings imply, do not oppose it. Lastly, 
tweets hashtagged with \#KickVic and \#IStandWithVic can share either political stance or as my results imply, cannot share both.

With this categorization in mind, it becomes possible to discuss how Twitter users subvert or perpetuate dominant ideologies in their tweets in consideration of the theoretical notions of CDS and FCDA. As a reminder, I view discursive constructions as patterns of how tweeters invoke a person, object, event, etc., in discourse through features, strategies, and representations. I examined these patterns with a few methods, such as the discursive strategies of nomination and predication, the social actor representations of collocations, role allocations, assimilation, and categorization, as well as the ideological square. Because we embed ideologies into discourse, and discourse is a representation of expressed meanings through forms, examining these patterns and their features allow me to understand how tweeters express their beliefs and values of gender ideologies and how the power asymmetries associated with these ideologies are subverted or perpetuated.

Although the affinity continuum notion forgoes social groups in favour of spaces, ideologies remain a part of this continuum because Twitter users bring ideological beliefs and values with them when they ambiently affiliate with others. One such example of this from my findings is that tweeters associated women in all the corpora with verbs that disempowered them because the qualities these actions are associated with are negative (Table 16), therefore reinforcing power asymmetries across this continuum. These theoretical considerations also help to explain the negative emphasis of the women's collective in comparison to other groups through ideological squaring. Taken together, the central notions of CDS and FCDA provide a set of approaches and ideas that allowed 
me to understand the implicit reinforcement of dominant ideologies within this affinity continuum. In summary, the theoretical notions of affinity space alongside ambient affiliation and digital activism demonstrate, from my findings, how tweeters use and situate these hashtags relating to the social issues of this specific instance of protest and counter-protest. Furthermore, the approaches of CDS and FCDA within this context illustrate how tweeters in my corpora discuss the content of this protest and counterprotest as well as how the patterns within this continuum either subvert or perpetuate oppressive gender ideologies related to women and sexual harassment allegations.

As the findings of this research demonstrate, tweeters both subvert and perpetuate patriarchal ideologies in this protest and counter-protest. While the protest met its goal in the form of subverting patriarchal ideologies to enact social change, the data that I have analyzed argues that there are also vast quantities of perpetuation in the tweets of the protest and counter-protest hashtags. Subversion exists in these tweets in the form of an empathetic discourse for victims (Example 26) and discourse of societal critique (Example 27).

Example 26: $[\ldots]$ but, heavens, how many women need to report that they were harassed before they are believed? \#KickVic

Example 27: [...] The Anime Community and the Nerd Community at large has humongous problems with rape culture, and it is time it stops. [hashtag removed] \#metoo \#kickvic

These examples highlight that by evoking the issue of sexual harassment and acknowledging it as a pervasive problem in society, tweeters that use \#KickVic in their posts subvert patriarchal ideologies. However, of the thirty-one tweets that I examined for 
the discursive constructions of women, only seven of them subverted these dominant ideologies. One interpretation of these findings is that from this small sample of data, Twitter users more frequently perpetuated patriarchal ideologies in their tweets. Additionally, these tweets come from the \#KickVic corpus, the \#IStandWithVic corpus, and the mixed corpus, meaning that perpetuation exists alongside subversion in the protest hashtag. Tweeters construct the perpetuation of these dominant ideologies through various discourses, like a discourse of male victimization (Palomino-Manjón, 2020; Examples 28-29) and a discourse of dissociation (Examples 30-31).

Example 28: Johnny and Vic Mignogna need to hang out sometime, they both got fucked out of their jobs by women who think everyone should just accept and believe them. [hashtag removed] \#IStandWithVic

Example 29: It's sad, I can't bring myself to accept Biden's newest accusers' claims, even though Biden's a known creeper. \#KickVic and Kavanaugh happened in the exact same way. [...]

Example 30: Don't speak for all women. Plenty of us are not as easily lead/persuadable as you lot. [...] \#IStandWithVic

Example 31: These women are terrible people, in reality a woman in that position (who were attacked before) would lash out after said kiss no questions asked. \#IStandWithVic

This discourse of male victimization may be explained by the idea that tweeters of the politically opposite stance of \#KickVic construct men as the victims of women's actions. The present results are consistent with Palomino-Manjón's (2020) work that studies the 
hashtag \#WhyIDidntReport. These results strongly imply that this discursive construction of women as the perpetrators, and men as the victims, perpetuates dominant ideologies by lamenting the loss of men's power and constructing this digital activism as the cause for why this happened. Additionally, this politically opposite stance argues that the protest challenging the patriarchal ideologies is the problem facing society rather than the dominant ideologies as the issue, resulting in further embedment of these beliefs. Secondly, the discourse of dissociation between women has a similar effect as women categorize themselves into groups according to how they challenge patriarchal ideologies. My findings highlight that by emphasizing this group according to their actions, tweeters separate women into those who are 'bad' because they use digital activism to challenge power asymmetries of gender ideologies and those who are 'good' because they do not seek to dispute these asymmetries with this method. This finding may be explained by the idea of the ideological square as this discourse constructs women who advocate for societal change as the outgroup and condemns them for these actions, resulting in the perpetuation of patriarchal ideologies associated with the counter-protest. Taken together, this consistent perpetuation of dominant ideologies in the protest and counter-protest may be one reason why, as time continued, there were fewer new tweets that used the \#KickVic hashtag as sexual harassment survivors found it exhausting to frequently encounter these oppressive structures.

These results strongly imply that perpetuations of oppressive patriarchal ideologies are frequent across my corpora, including tweeters that hijacked the \#KickVic hashtag or perpetuated their patriarchal ideology by using both hashtags together. Despite these results, it is crucial to remember their context and the success of \#KickVic's protest 
within this perpetuation. Recalling the size of the corpora, the \#IStandWithVic corpus contains nearly double the number of tweets in the \#KickVic corpus. Nevertheless, I observe the success of the \#KickVic protest in the form of two companies firing Mignogna and several conventions removing him from their guest line-up. The theoretical takeaway I want to emphasize here is that the frequency of a hashtag does not equate to "success" and that higher frequency does not equal meaningfulness. While more tweets contributing to the protest is an affordance of hashtags used in the service of digital activism, higher frequency does not mean that (counter-)protest will realize success. In my view, success for digital activism means the critiquing of power asymmetries and dominant ideologies in a way that makes stakeholders listen and demonstrating that these stakeholders have the potential to initiate social change through their position. Anime fans and stakeholders exist together within affinity spaces on the Internet as they both heavily rely on each other for support. With the growing rumours of Mignogna's allegations over the years, the hashtag — a resource that draws users together and creates a space for discussion and affiliation — acted as the tool to bring advocates together in a way that would garner the stakeholders' attention and encourage them to lead social change. This case study concludes that numbers can be meaningful for digital activism, but success comes from effectively creating social change for the betterment of society.

Importantly, this thesis does not attempt to measure the success of this digital activism. However, some studies suggest how tweeters can use hashtags to successfully advance their advocacy (Saxton et al., 2015) and how hashtags recirculate in other contexts to "[interact] with and [have] consequences on public debate more generally" 
(Barker-Plummer \& Barker Plummer, 2017, p. 15). While the meaning of success can be determined for digital activism because activism has a determined goal of enacting social change, I believe it is more difficult to measure the success of \#KickVic. On top of a corpus constructed from \#KickVic tweets, such a study would require survey data from tweeters of the hashtag to understand why they used it for digital activism. These factors by no means make a study of this type impossible. Instead, measuring "success" was not in the original plan for this thesis and is more work than I believe one researcher can complete.

\subsection{Summary}

In this chapter, I used the theoretical considerations that inform my study to understand the results from my analysis and to answer the research questions that guide this project. Firstly, I summarize why the theoretical concepts and methodologies of this study occupy a research gap and then answer the three research questions in sequential order. The design of the corpora aimed to understand the similarities and differences between them, and the research questions address this. Secondly, the results of this research provide supporting evidence that the corpora discuss the same issues but how tweeters discuss these issues varies according to the corpus. These results strongly imply that there is fluidity in the discursive constructions, and this fluidity exists between the corpora and within the protest and counter-protest. Thirdly, my findings highlight that in the tweets that include women, tweeters often collectivize this social actor and discursively construct them with negative lexical and othering strategies. As well, this study finds that the protest (\#KickVic) involves those that agree with its political stance as well as those who oppose it and the counter-protest (\#IStandWithVic) includes only those 
tweeters who follow this hashtag's stance. Additionally, I found individuals who subvert patriarchal ideologies and tweeters who perpetuate them throughout the protest and counter-protest affinity continuum, implying that the continuum does not restrict which hashtag they can use. Following this, I summarize the content of this project and provide future research directions in the final chapter of this thesis. 


\section{Chapter 6: Conclusion}

In this chapter, I summarize this thesis, beginning with its inspiration and concluding with its implications. Additionally, I highlight this study's limitations and, as CDS research stipulates, critique my work with a critical eye. Lastly, I describe areas this project illuminates for future research directions, including future theoretical and methodological considerations.

\subsection{Summary}

In its conception, this research began as part of my passion to understand how Twitter users discursively construct this instance of digital activism because the affinity continuum it encompasses is relevant to my life. Guided by three research questions, this thesis investigated the similarities and differences of the hashtags \#KickVic and \#IStandWithVic to reveal how Twitter users discursively construct this protest and counter-protest as well as the social actor of women. Given that these hashtags represent a salient case of digital activism on social media, I also found it necessary to examine the extent to which these hashtags perpetuate or subvert gendered powered asymmetries that manifest through patriarchal ideologies. Even though this research explores only one instance of hashtags used in the service of digital activism on Twitter, it fills a unique research gap thanks to its theoretical and methodological considerations. As such, while the findings of this research cannot be generalized to every instance of hashtags used for this purpose, it does provide future insights as to what other scholars might observe in similar contexts.

One aspect that makes this research unique compared to other studies that have examined hashtags used for creating awareness concerning sexual harassment is the 
theoretical conception of hashtags as affinity spaces (Gee, 2005). Constructed from the need to describe groups of people without using the word 'community' due to its complicated interpretations, Gee (2005) purposes the concept of a social semiotic space (SSS). Concerned with a particular type of SSS, Gee (2005) also defines the idea of an affinity space by a set of eleven features. Under this notion, hashtags are portals into a larger affinity space where users interact thanks to the overarching topic of shared interest. In this space, users affiliate ambiently with others that share their values (Zappavigna, 2011), which can result in the hashtag also serving as a marker for those values. Additionally, Gee (2005) notes that social activists use affinity spaces to critique social issues and advocate for social change. As such, this study adopts the approaches of CDS and FCDA to investigate the discursive constructions in this protest and counterprotest. For my project, CDS addresses the related theoretical notions of discourse, ideology, and power, and FCDA contributes a political perspective that allows for an investigation of power asymmetries that subvert or perpetuate oppressive gender ideologies.

However, while the methodologies of these two approaches allow for an in-depth, qualitative analysis of the data, they both have their limitations. As such, this thesis also draws on a corpus linguistics methodology and combines the synergy of these approaches to mitigate these limitations. Utilizing the CL statistical procedures to generate an overview of the data and pinpoint areas for down-sampled CDS/FCDA analysis, this project analyzed three corpora I constructed around the protest and counter-protest hashtags. This analysis entailed analyzing raw and normalized word frequencies, similarities and differences for semantic themes and keywords, and a down-sampled 
investigation of the social actor women in terms of collocates and representations related to role allocation, assimilation, collectivization, and Us versus Them. Following analysis, I interpreted the findings to understand how tweeters discursively constructed this protest, counter-protest, and women in this protest and counter-protest with relation to the context and the theoretical concepts that ground this study.

During analysis, a few recurring results emerged. Firstly, words referring to men were far more frequent across all three corpora than those referencing women after I determined the normalized word frequencies. Additionally, I observed this finding when comparing the \#KickVic corpus and the \#IStandWithVic corpus against the mixed reference corpus as three out of the four similar keywords they shared referred to men (he, him, and his). Secondly, a recurring finding from the similarities and differences analysis is that the three corpora talk about the same issues, as demonstrated by the semantic themes I identified, and tweeters construct these issues differently at the lexical level, as the unique keywords illustrate. Furthermore, my findings provide supporting evidence that the \#KickVic corpus and the \#IStandWithVic corpus are the least similar while each of these corpora shares similarities with the mixed corpus. This idea is further supported by the finding from the direct corpus comparison that the \#KickVic corpus is the most different of the three corpora. Lastly, my results from the discursive construction of social actor representations illustrate that tweeters often discuss the social actor of women in terms of their victimhood, collectivization, and negative othering.

Admittedly, these procedures generated many more results than I had time to interpret. However, this overabundance did not prevent me from answering my research questions. Firstly, this research discovers that the tweeters of the protest and counter- 
protest hashtags discursively construct these two sides as both similar and different from one another. My findings support this interpretation as I found Twitter users talk about the same issues in tweets that contain \#KickVic and \#IStandWithVic but they use different constructions to represent these issues. Additionally, I discovered discursive constructions that delegitimize the \#KickVic protest in tweets that use \#IStandWithVic and tweets that use the protest hashtag. This finding recurs relating to the second research question, as the social actor of women is both positively and negatively discursively constructed in the protest hashtag. However, despite the successful protest, tweeters more frequently discursive construct women as the issue that society needs to protect itself from, especially in the \#IStandWithVic corpus and the mixed corpus.

Overall, this thesis finds that Twitter users both subvert and perpetuate oppressive patriarchal ideologies in the affinity continuum of this protest and counter-protest. Additionally, I obtained evidence that discourses which perpetuate power asymmetries and gender ideologies were more frequent across the three corpora in consideration of those tweets that were a part of the down-sampled analysis. However, I cannot generalize these findings and interpretations to every instance of a hashtag used in the service of digital activism. Despite this, my research exemplifies the fluidity that can exist between a protest and counter-protest hashtag. Furthermore, this fluidity seems to be used more so by those Twitter users who hold an ostensibly politically opposite stance in comparison to the protest.

\subsection{Limitations}

There are a few limitations concerning the contents of this study. Some of these limitations I encountered during corpus construction and certain limitations are related to 
the results. Firstly, due to the sheer amount of data I collected, the three corpora I constructed for this project are imperfect and could undergo further cleaning procedures to make corpus linguistics analysis easier. Even though I cleaned and formatted the corpora to the best of my ability given the time constraints, I discovered errors, like random strings of characters, in concordance lines while using AntConc. Furthermore, I noticed variations in the hashtags \#KickVic and \#IStandWithVic after I completed corpus construction and began data analysis. For example, the \#KickVic corpus is not made of tweets without the \#IStandWithVic hashtag because some tweets contain the abbreviated hashtag $\# I S W V$. I feel that further research examining these hashtags can mitigate this issue by resorting the tweets more carefully in consideration of synonymous hashtags.

A second limitation of this study is that it was infeasible to investigate every avenue that the word frequencies, semantic themes, and keywords analysis highlighted as potential areas for further investigation. This limitation is due to time constraints and the fact I completed this analysis by myself. As such, the findings, and my interpretations of them, are informed by what I discovered and not what exists in the corpora that I did not have the opportunity to analyze. For example, this thesis does not consider the importance of multi-modality to social media data, a significant line of questioning that scholars have explored with critical discourse analysis (Machin \& Mayr, 2012) and social media critical discourse analysis (KhosraviNik, 2017).

There are also a few limitations to address concerning the semantic themes/keywords analysis and the social actor representation analysis of this research could have been enhanced in several ways. Firstly, my analysis of similar keywords between the corpora contains potential issues as my corpora sharing a keyword does not 
mean that tweeters use this keyword in the same ways. The down-sampled analysis of women (Section 4.4) illustrates this truth. In the future, I will implement a more rigorous analysis of keyword collocates and concordances to determine if and how tweeters use these shared keywords in their respective corpora to determine semantic themes.

Secondly, had time been available, I could have involved a second coder in the project. With this addition, the analysis could undergo a more rigorous and enhanced process through multiple perspectives and intercoder reliability.

Although the present study uses three corpora constructed from 27,527 tweets of this protest and counter-protest, it is appropriate to recognize that my findings cannot be generalized for the entire protest and counter-protest. Additionally, this is true for the 'peak activity' of this affinity continuum, which is also difficult to define. This inability to generalize is because portions of time are missing from my corpora, and tweeters continue to use these hashtags amidst writing this thesis. From these limitations, I acknowledge there is still work to be done with these data to understand fully and even considering this possibility the findings may still not be generalizable. However, this does not detract from the reality that, thanks to the attentive procedures I followed, I identified significant and recurring patterns in the discursive constructions that revealed gendered power asymmetries that entrench patriarchal ideologies in this protest and counterprotest.

\subsection{Future research directions}

As the previous section highlights, there are many avenues for future research, both with these data and other research projects. In addition to those already mentioned, I suggest several avenues of research that may be helpful. Firstly, these data can be 
explored in terms of social actors, like Mignogna, to understand how they are discursively constructed and create a point of comparison between the social actor of women. Additionally, I could examine every instance of women across all three corpora using social actor representations to produce a more robust understanding of how tweeters discursively constructed them in this affinity continuum. Future research could also examine multimodal elements of this corpus, as related to social actors or other areas, to understand how emojis, images, videos, and hyperlinks contribute to discourses that subvert or perpetuate patriarchal ideologies. Furthermore, there is potential to investigate how hashtags pattern together in these corpora to reveal the extent to which tweeters use other affinity spaces in conjunction with this pair of hashtags and if/how they are related to the subversion or perpetuation of ideologies. Lastly, future work could analyze the tweets from each corpus that Twitter users have retweeted the most to discern how these frequently shared messages discursively construct the protest and counterprotest and what political stances tweeters are distributing across the platform. With the size of these corpora, the potential research possibilities within them are nearly endless.

In terms of future research, some avenues involve not only this dataset. For example, I could compare these three corpora against a reference corpus comprised of tweets from other hashtags used in the service of digital activism to examine what elements of this discourse are specific to the \#KickVic and \#IStandWithVic hashtags. Additionally, my research illustrates how scholars can ethically construct the hashtagged tweets of protests and counter-protests into individual corpora in ways that allow for the comparison of similarities and differences between these affinity spaces. Though I could arrange the tweets of these hashtags into one corpus, the findings from this research 
demonstrate that there are benefits to separating protest and counter-protest hashtags into separate corpora, such as examining what discourses in each subvert or perpetuate dominant ideologies. In my view, the most compelling finding of this research is that it is meaningful to investigate a digital protest attributed to a niche interest (the NA voice acting industry and anime conventions) for these power asymmetries and gender ideologies. Digital protests are not valueless. Instead, the continued perpetuation of oppressive patriarchal ideologies in the original protest hashtag provides evidence as to why survivors of sexual harassment use this resource to safely advocate for societal transformation. 


\section{Appendices}

\section{Appendix A Research questions}

\begin{tabular}{|c|c|c|c|}
\hline \multicolumn{4}{|c|}{$\begin{array}{l}\text { RQ1. 1. How do the similarities and differences of keywords and semantic themes } \\
\text { discursively construct the protest and counter-protest? }\end{array}$} \\
\hline Identify patterns & Sub-questions & Methods & Supporting theory \\
\hline \multirow{3}{*}{$\begin{array}{l}\text { Are the word } \\
\text { frequencies, } \\
\text { semantic themes, } \\
\text { and keywords } \\
\text { similar and/or } \\
\text { different in the } \\
\text { corpora? }\end{array}$} & $\begin{array}{l}\text { What semantic } \\
\text { themes/keywords } \\
\text { does the \#KickVic } \\
\text { corpus discuss? }\end{array}$ & $\begin{array}{l}\text {-Keywords of topics, } \\
\text { actors, actions, } \\
\text { attributes }\end{array}$ & \multirow{3}{*}{$\begin{array}{l}\text {-Keywords show } \\
\text { 'unique' items } \\
\text { (against reference } \\
\text { corpus) } \\
\text {-Compare semantic } \\
\text { themes/keywords of } \\
\text { three corpora to } \\
\text { examine how they } \\
\text { are similar/different } \\
\text { to general written } \\
\text { English and each } \\
\text { other }\end{array}$} \\
\hline & $\begin{array}{l}\text { What semantic } \\
\text { themes/keywords } \\
\text { does the } \\
\text { \#IStandWithVic } \\
\text { corpus discuss? }\end{array}$ & $\begin{array}{l}\text {-Keywords of topics, } \\
\text { actors, actions, } \\
\text { attributes }\end{array}$ & \\
\hline & $\begin{array}{l}\text { What semantic } \\
\text { themes/keywords } \\
\text { does the mixed } \\
\text { corpus discuss? }\end{array}$ & $\begin{array}{l}\text {-Keywords of topics, } \\
\text { actors, actions, } \\
\text { attributes }\end{array}$ & \\
\hline \multicolumn{4}{|c|}{ RQ2. How do Twitter users discursively construct women in this protest and counter-protest? } \\
\hline Identify patterns & Sub-questions & Methods & Supporting theory \\
\hline \multirow[t]{3}{*}{$\begin{array}{l}\text { Are the discursive } \\
\text { constructions of } \\
\text { women similar } \\
\text { and/or different in } \\
\text { the corpora? }\end{array}$} & $\begin{array}{l}\text { What discursive } \\
\text { constructions do } \\
\text { tweeters use in the } \\
\text { \#KickVic corpus? }\end{array}$ & $\begin{array}{l}\text {-Discursive } \\
\text { representations of } \\
\text { social actors (role } \\
\text { allocation, nomination, } \\
\text { assimilation) }\end{array}$ & \multirow{3}{*}{$\begin{array}{l}\text { Lazar: gender } \\
\text { ideology/relations } \\
\text { of power are } \\
\text { "produced, } \\
\text { sustained, } \\
\text { negotiated, and } \\
\text { challenged" in } \\
\text { discursive } \\
\text { constructions } \\
(2007, \text { p. 142). }\end{array}$} \\
\hline & $\begin{array}{l}\text { What discursive } \\
\text { constructions do } \\
\text { tweeters use in the } \\
\text { \#IStandWithVic } \\
\text { corpus? }\end{array}$ & $\begin{array}{l}\text {-Discursive } \\
\text { representations of } \\
\text { social actors (role } \\
\text { allocation, nomination, } \\
\text { assimilation) }\end{array}$ & \\
\hline & $\begin{array}{l}\text { What discursive } \\
\text { constructions do } \\
\text { tweeters use in the } \\
\text { mixed corpus? }\end{array}$ & $\begin{array}{l}\text {-Discursive } \\
\text { representations of } \\
\text { social actors (role } \\
\text { allocation, nomination, } \\
\text { assimilation) }\end{array}$ & \\
\hline \multicolumn{4}{|c|}{$\begin{array}{l}\text { RQ3. To what extent do these findings subvert or perpetuate (i) dominant ideologies related } \\
\text { to sexual harassment and (ii) digital activism hashtags to address this social issue? }\end{array}$} \\
\hline \multicolumn{2}{|c|}{ Methods } & \multicolumn{2}{|c|}{ Supporting theory } \\
\hline \multicolumn{2}{|c|}{$\begin{array}{l}\text { Meta-analysis and comparison of findings } \\
\text { from RQ1 and RQ2 }\end{array}$} & \multicolumn{2}{|c|}{$\begin{array}{l}\text { Expands upon the understanding of } \\
\text { discursive constructions of digital protests } \\
\text { and counter-protests }\end{array}$} \\
\hline
\end{tabular}




\section{Appendix B Data cleaning and sorting procedures}

Although I knew before beginning the data cleaning/sorting process that I would encounter issues, I faced several problems while working with my data that I was not prepared for. The most frustrating issue is that, depending on the data type, Microsoft Excel does not consistently show duplicates when using the highlight duplicates function. By duplicates, I mean tweets from the same user that the scraping tools mistakenly archived twice in the spreadsheet. I deleted these duplicates because I did not want them to skew word frequency counts, keywords, collocates, or concordances. For what I assume is because Excel is more suited to dealing with numbers or one string of characters per cell rather than text, I noticed that the software highlighted some duplicate tweets but not others. As such, it is not a reliable method to determine duplicates via only the text column (the column that contains the text of the tweet) in the spreadsheet. With the collection tools I used, the tool designated one column of the spreadsheet for each type of metadata about the tweet like text, tweeter's username, the status URL of the tweet, and the timestamp of the tweet, while each row contained all the information for one tweet. To circumvent this issue, I used the status URL column to verify tweet duplication as a status URL is unique to every tweet (no two status URLs are the same) and it is always a singular string of characters without breaks. Using this method, Excel consistently identified cells that contained the same status URL, and I easily deleted the duplicate instances of a tweet by removing the entire row that it was contained in.

Another issue I encountered while cleaning the data was that TwitterScraper saved certain characters - like punctuation - in a different encoding. After two months of searching the web, I determined that this encoding was ISO/IEC 8859-1, a character- 
encoding scheme developed in the 1980s. I am unsure why TwitterScraper converted certain characters into this encoding, but I found a legend (Starr, 2017) that showed the equivalent of the ISO/IEC 8859-1 character set alongside the UTF-8 one. With this information, I used the Find and Replace function in Excel to convert the strings of characters back into UTF-8 to ensure corpora consistency. 


\section{Appendix C Analysis of similarities and differences}

The following tables illustrate all of the semantic themes and keywords produced from the similarities and differences comparisons. Tables are named according to the procedure they were a result of.

\section{C.1 Keyword similarities}

Table C.1.1: AmE06/BE06 comparison: Shared between \#KickVic and \#IStandWithVic

\begin{tabular}{|c|c|c|}
\hline Semantic theme & All keywords of semantic theme & Total keywords \\
\hline References to Mignogna & $\begin{array}{l}\text { vic, mignogna, he’s, vic's, him, } \\
\text { @vicmignogna }\end{array}$ & 6 \\
\hline Evidence & evidence, video, accusations, proof, pic & 5 \\
\hline Groups of people & $\begin{array}{l}\text { funimation,@funimation,@youtube, } \\
\text { fans,guys, people }\end{array}$ & 6 \\
\hline (Affinity) Spaces & con,twitter,@youtube & $3{\left.\text { (2 } \text { unique }^{6}\right)}$ \\
\hline Attributes & innocent, guilty & 2 \\
\hline Pieces of web addresses & $\begin{array}{l}\text { youtube,www,@youtube, via, https, } \\
\text { com, twitter, status, pic, anime, youtu, } \\
\text { co }\end{array}$ & 12 (9 unique) \\
\hline A collective & all & 1 \\
\hline Specified individuals & $\begin{array}{l}\text { individual_A, someone, funimation, } \\
\text { @,funimation,@youtube, } \\
\text { @individual_A, anyone }\end{array}$ & 7 (4 unique) \\
\hline Actions & know, believe, say, want, think, prove & 6 \\
\hline
\end{tabular}

\footnotetext{
${ }^{6}$ Some keywords belong to more than one semantic theme. For determining totals, 'unique' means that the number of keywords in brackets has not yet been categorized under a semantic theme.
} 
Table C.1.2: AmE06/BE06 comparison: Shared between \#KickVic and mixed

\begin{tabular}{|c|c|c|}
\hline Semantic theme & All keywords of semantic theme & Total keywords \\
\hline References to Mignogna & $\begin{array}{l}\text { vic, mignogna, vic’s, @vicmignogna, } \\
\text { he’s }\end{array}$ & 5 \\
\hline Evidence & $\begin{array}{l}\text { pic, evidence, proof, accusations, } \\
\text { allegations, video }\end{array}$ & 6 \\
\hline Groups of people & $\begin{array}{l}\text { funimation,@funimation,@youtube, } \\
\text { fans, guys, people, vas, side, victims, } \\
\text { supporters }\end{array}$ & 10 \\
\hline Protest & thing & 1 \\
\hline (Affinity) Spaces & con, cons, twitter,@youtube,\#kickvic & 5 (4 unique) \\
\hline Attributes & innocent, guilty, fake & 3 \\
\hline Harassment & harassment & 1 \\
\hline Pieces of web addresses & $\begin{array}{l}\text { youtube,@youtube, twitter, pic, www, } \\
\text { via, https, com, status, anime, youtu, co }\end{array}$ & 12 (9 unique) \\
\hline A collective & all, whole & 2 \\
\hline Specified individuals & $\begin{array}{l}\text { individual_A, funimation, } \\
\text { @,funimation,@youtube, } \\
\text { @individual_A, anyone }\end{array}$ & 6 (3 unique) \\
\hline Actions & $\begin{array}{l}\text { know, believe, want, think, prove, hate, } \\
\text { say }\end{array}$ & 7 \\
\hline
\end{tabular}


Table C.1.3: AmE06/BE06 comparison: Shared between \#IStandWithVic and mixed

\begin{tabular}{lll}
\hline Semantic theme & All keywords of semantic theme & Total keywords \\
\hline References to Mignogna & $\begin{array}{l}\text { vic, mignogna, vic's, @vicmignogna, } \\
\text { he's, \#vicmignogna }\end{array}$ & 6 \\
Evidence & pic, evidence, proof, accusations, video & 5 \\
funimation, @funimation, @youtube, & 7 \\
fans, guys, people, \#westandwithvic & \\
con, twitter, @youtube, \#istandwithvic, 12 (10 unique) \\
\#vicmignogna, \#vickicksback, \#iswv, \\
\#kickvic, \#westandwithvic, \#weebwars, \\
\#fearthebeard, \#animegate \\
innocent, guilty \\
Aoutube,@youtube, twitter, pic, www, \\
Pieces of web addresses \\
$\begin{array}{l}\text { via, https, com, status, anime, youtu, } \\
\text { co, gofundme }\end{array}$
\end{tabular}

Table C.1.4: Mixed corpus as reference: Keywords shared between \#KickVic and \#IStandWithVic

\begin{tabular}{lll}
\hline Class $^{7}$ & All keywords of class & Total shared keywords \\
\hline Pronouns & he, him, his, you & 4 \\
\hline
\end{tabular}

\footnotetext{
${ }^{7}$ Due to the small number of common words with this comparison, I categorized these words by a class
} rather than a theme. 
Table C.1.5: \#IStandWithVic corpus as reference: Keywords shared between \#KickVic and mixed

\begin{tabular}{lll}
\hline Semantic theme & All keywords of class & Total shared keywords \\
\hline (Affinity) Spaces & $\begin{array}{l}\text { \#kickvic, \#kickvic's, \#metoo, } \\
\text { \#fuckvic }\end{array}$ & 4 \\
Groups of people & $\begin{array}{l}\text { victims, side, people, } \\
\text { supporters, sides } \\
\text { movement, thing }\end{array}$ & 5 \\
The protest & whole & 2 \\
A collective & @individual_B & 1 \\
Specified individuals & swat, involve & 1 \\
Actions & 2 \\
\hline
\end{tabular}

Table C.1.6: \#KickVic corpus as reference: Keywords shared between \#IStandWithVic and mixed

\begin{tabular}{|c|c|c|}
\hline Semantic theme & All keywords of class & Total shared keywords \\
\hline Reference to Mignogna & \#vicmignogna, vic & 2 \\
\hline (Affinity) Spaces & $\begin{array}{l}\text { \#istandwithvic, \#vickicksback, } \\
\text { \#westandwithvic, } \\
\text { \#fearthebeard, \#iswv, } \\
\text { \#weebwars, \#women4vic, } \\
\text { \#boycottfunimation, } \\
\text { \#istandwithindividual_F, } \\
\text { \#vicmignogna, \#animegate, } \\
\text { \#fireindividual_A, } \\
\text { \#kamehacon, \#individual_A, } \\
\text { \#innocentuntilprovenguilty, } \\
\text { \#funimation }\end{array}$ & 16 (15 unique) \\
\hline Groups of people & \#westandwithvic, \#women4vic & 2 (0 unique) \\
\hline Piece of web addresses & $\begin{array}{l}\mathrm{t}, \mathrm{co} \text {, https, com, twitter, } \\
\text { gofundme, www, youtu, watch }\end{array}$ & 9 \\
\hline Specified individuals & $\begin{array}{l}\text { individual_D, individual_C, } \\
\text { @individual_E, } \\
\text { \#individual_A, } \\
\text { @individual_G, individual_H }\end{array}$ & 6 (5 unique) \\
\hline Actions & kick $\quad-$ & 1 \\
\hline
\end{tabular}




\section{C.2 Keyword differences}

Table C.2.1: AmE06/BE06 comparison: Distinct to \#KickVic

\begin{tabular}{llll}
\hline (Affinity) Spaces & Harassment & Actions/attributes & People \\
\hline movement (286) & sexual (278) & fucking (137) & they're (190) \\
hashtag (152) & voice (270) & saying (214) & them (592) \\
\#standwithvic (138) & claims (203) & wrong (200) & individual_B \\
& & & \\
& stories (187) & & \\
& assault (123) & & \\
\hline
\end{tabular}

Table C.2.2: AmE06/BE06 comparison: Distinct to \#IStandWithVic

\begin{tabular}{lll}
\hline (Affinity) Spaces & Actions/attributes & People \\
\hline kv (257) & love (598) & @individual_I (495) \\
\#women4individual_F (1) & kicks (308) & @individual_J (343) \\
\#women4vic (158) & support (542) & @individual_K (337) \\
\#women (1) & stand (387) & broly (293) \\
& hope (413) & @individual_Emedia (264) \\
& thank (304) & @individual_L (255) \\
blocked (229) & @individual_M (250) \\
& see (693) & followers (202) \\
& & vicmignogna (191) \\
& & person (394) \\
\hline
\end{tabular}


Table C.2.3: AmE06/BE06 comparison: Distinct to mixed

\begin{tabular}{lllll}
\hline $\begin{array}{l}\text { (Affinity) } \\
\text { Spaces }\end{array}$ & Discourse & The protest & Actions/attributes & People \\
\hline \#anime (170) & $\begin{array}{l}\text { tweet } \\
(89)\end{array}$ & $\begin{array}{l}\text { situation } \\
(195)\end{array}$ & false (118) & \#individual_A \\
\#individual_A & $\begin{array}{l}\text { tweets } \\
(83)\end{array}$ & vs (115) & tweet (89) & \#funimation (107) \\
$\begin{array}{l}\text { (109) } \\
\text { \#kamehacon }\end{array}$ & & sides (135) & let's (77) & @individual_C \\
$\begin{array}{l}\text { (108) } \\
\text { \#funimation }\end{array}$ & & & & (84) \\
$\begin{array}{l}\text { (107) } \\
\text { \#bethere (83) }\end{array}$ & & & & \\
\hline
\end{tabular}


Table C.2.4: Corpora comparison: Distinct to \#KickVic

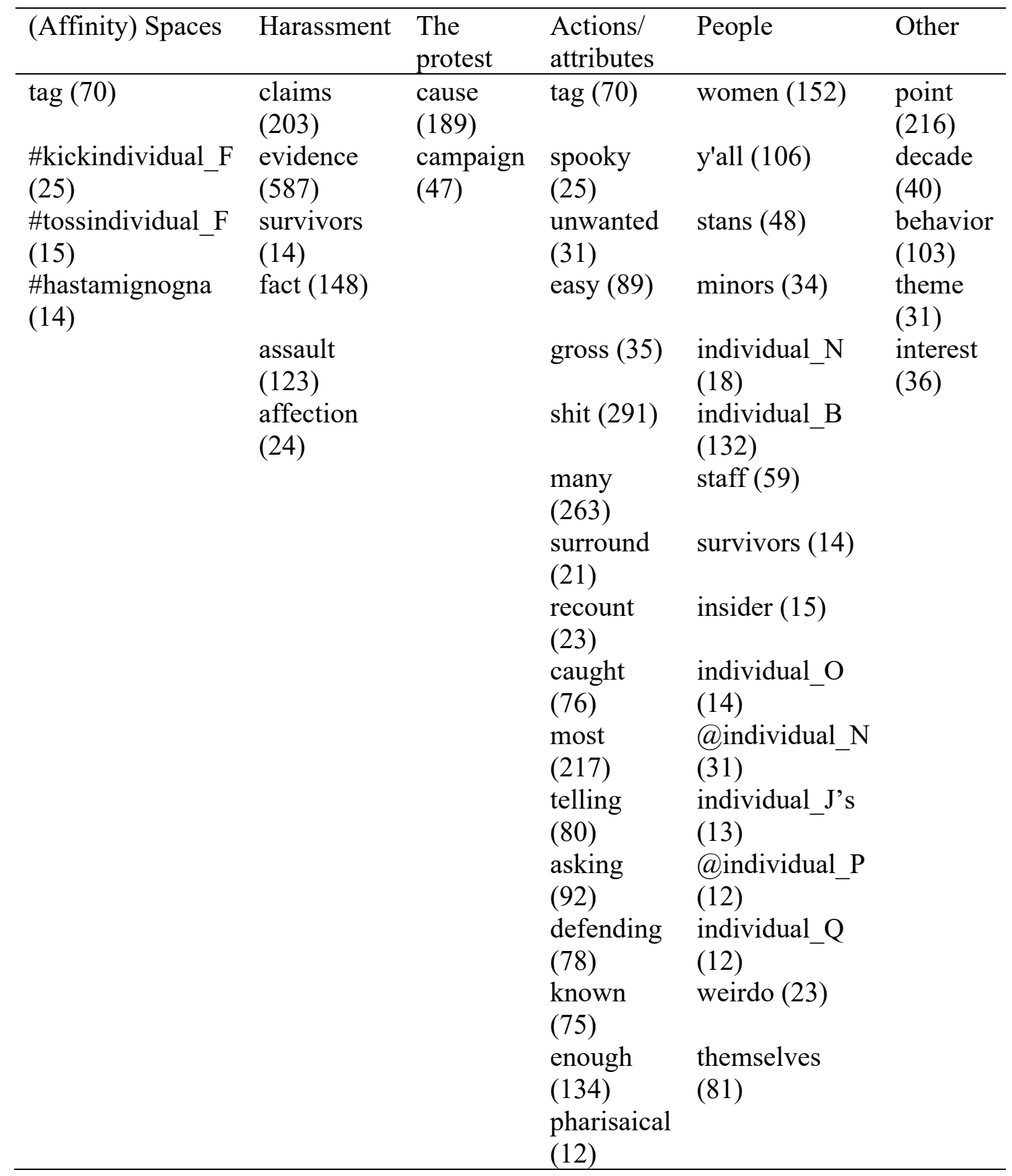


Table C.2.5: Corpora comparison: Distinct to \#IStandWithVic

\begin{tabular}{|c|c|c|c|c|c|}
\hline (Affinity) Spaces & Harassment & $\begin{array}{l}\text { The } \\
\text { protest }\end{array}$ & $\begin{array}{l}\text { Actions/ } \\
\text { attributes }\end{array}$ & People & Other \\
\hline \#funimationboycott (38) & \multirow[t]{16}{*}{ hug (82) } & \multirow[t]{16}{*}{$\begin{array}{l}\text { petition } \\
(68)\end{array}$} & $\begin{array}{l}\text { wonderful } \\
\text { (64) }\end{array}$ & $\begin{array}{l}\text { @individual_R } \\
\text { (34) }\end{array}$ & $\begin{array}{l}\text { redbubble } \\
\text { (39) }\end{array}$ \\
\hline \#beavoiceforgood (33) & & & met (153) & $\begin{array}{l}\text { @individual_s } \\
(48)\end{array}$ & PST (27) \\
\hline \#welovevic (30) & & & proper (69) & fellow (70) & \\
\hline \#vicisback (28) & & & $\begin{array}{l}\text { discovery } \\
(56)\end{array}$ & we (1439) & \\
\hline \#broly (56) & & & $\operatorname{good}(545)$ & $\begin{array}{l}\text { @ funimation } \\
\text { (387) }\end{array}$ & \\
\hline \#boycottroosterteeth (26) & & & hug (82) & @anime (92) & \\
\hline \#walkaway (26) & & & donate (94) & defendant (52) & \\
\hline \multirow{9}{*}{$\begin{array}{l}\text { \#istandwithindividual_E } \\
\text { (25) }\end{array}$} & & & please & funimation (404) & \\
\hline & & & $(438)$ & & \\
\hline & & & sign (96) & $\begin{array}{l}\text { individual_A } \\
\text { (514) }\end{array}$ & \\
\hline & & & awesome & @individual_T & \\
\hline & & & $(96)$ & (30) & \\
\hline & & & $\begin{array}{l}\text { beautiful } \\
\text { (51) }\end{array}$ & \#broly (56) & \\
\hline & & & pray (29) & $\begin{array}{l}\text { @individual_U } \\
\text { (40) }\end{array}$ & \\
\hline & & & $\begin{array}{l}\text { positivity } \\
\text { (33) }\end{array}$ & her $(710)$ & \\
\hline & & & $\begin{array}{l}\text { rehire }(25) \\
\text { stay }(168)\end{array}$ & \#welovevic (30) & \\
\hline
\end{tabular}


Table C.2.6: Corpora comparison: Distinct to mixed corpus

\begin{tabular}{|c|c|c|c|c|}
\hline (Affinity) Spaces & Harassment & The protest & $\begin{array}{l}\text { Actions/ } \\
\text { attributes }\end{array}$ & People \\
\hline $\begin{array}{l}\text { \#kickindividual_A } \\
\text { (55) }\end{array}$ & $\begin{array}{l}\text { \#evidencematters } \\
\text { (13) }\end{array}$ & war $(50)$ & $\begin{array}{l}\text { neutral } \\
(68)\end{array}$ & \#npcs (22) \\
\hline $\begin{array}{l}\text { \#kickindividual_C } \\
\text { (25) } \\
\text { \#comicsgate }(51)\end{array}$ & $\begin{array}{l}\text { attacks (40) } \\
\text { controversy (33) }\end{array}$ & $\begin{array}{l}\text { controversy } \\
(33)\end{array}$ & $\begin{array}{l}\text { exposed } \\
(51)\end{array}$ & $\begin{array}{l}\text { @individual_A } \\
\text { (131) } \\
\text { @individual_C } \\
\text { (84) }\end{array}$ \\
\hline \#jellybean (14) & & & & supporter (59) \\
\hline \#jellybeanlivesmatter & & & & @individual_V \\
\hline$(14)$ & & & & (14) \\
\hline \#evidencematters (13) & & & & \\
\hline \#npcs (22) & & & & \\
\hline \#individual_Etheknife & & & & \\
\hline (12) & & & & \\
\hline
\end{tabular}




\section{References}

Anthony, L. (2020). AntConc (Version 3.5.9) [Computer Software]. Tokyo, Japan: Waseda University. Available from https://www.laurenceanthony.net/software

Baker, P. (2006). Using corpora in discourse analysis. Continuum.

Baker, P. (2009). The BE06 corpus of British English and recent language change. International Journal of Corpus Linguistics, 14(3), 312-337. https://doi.org/10.1075/ijcl.14.3.02bak

Baker, P. (2010). Sociolinguistics and corpus linguistics. Edinburgh University Press.

Baker, P., Gabrielatos, C., KhosraviNik, M., Krzyżanowski, M., McEnery, T., \& Wodak, R. (2008). A useful methodological synergy? Combining critical discourse analysis and corpus linguistics to examine discourses of refugees and asylum seekers in the UK press. Discourse \& Society, 19(3), 273-306. https://doi.org/10.1177/0957926508088962

Baker, P., \& Levon, E. (2015). Picking the right cherries? A comparison of corpus-based and qualitative analyses of news articles about masculinity. Discourse \& Communication, 9(2), 221-236. https://doi.org/10.1177/1750481314568542

Baker, P., \& McGlashan, M. (2020). Critical discourse analysis. In S. Adolphs \& D. Knight (Eds.), The Routledge handbook of English language and digital humanities (1st ed., pp. 220-241). Routledge. https://doi.org/10.4324/9781003031758

Barker-Plummer, B., \& Barker-Plummer, D. (2017). Twitter as a feminist resource: \#YesAllWomen, digital platforms, and discursive social change. In J. Earl \& D. 
A. Rohlinger (Eds.), Social movements and media: Studies in media and communications (Vol. 14, pp. 91-118). Emerald Publishing Limited. https://doi.org/10.1108/S2050-206020170000014010

Biber, D., \& Reppen, R. (2015). Introduction. In D. Biber \& R. Reppen (Eds.), The Cambridge handbook of English corpus linguistics (pp. 1-8). Cambridge University Press. https://doi.org/10.1017/CBO9781139764377.001

Bonilla, Y., \& Rosa, J. (2015). \#Ferguson: Digital protest, hashtag ethnography, and the racial politics of social media in the United States. American Ethnologist, 42(1), 4-17. https://doi.org/10.1111/amet.12112

Canadian Women's Foundation. (2021, June 9). Sexual assault and harassment in Canada: The facts. Retrieved July 20, 2021, from https://canadianwomen.org/the-facts/sexual-assault-harassment/

Clark, R. (2016). "Hope in a hashtag": The discursive activism of \#WhyIStayed. Feminist Media Studies, 16(5), 788-804. https://doi.org/10.1080/14680777.2016.1138235

D'Ambrosio, A. (2019). \#BlackLivesMatter and hashtag activism in a critical discourse analysis perspective. Quaderni del CeSLiC. Retrieved March 11, 2020, from http://amsacta.unibo.it/6177/

Duguid, A., \& Partington, A. (2018). Absence: You don't know what you're missing. Or do you? In C. Taylor \& A. Marchi (Eds.), Corpus approaches to discourse: A critical review (1st ed., pp. 38-59). Routledge.

Eggins, S. (2004). An introduction to systemic functional linguistics (2nd ed.). Continuum. 
Evans, A. (2016). Stance and identity in Twitter hashtags. Language@Internet, 13(1). Retrieved June 22, 2021, from https://nbn-resolving.org/urn:nbn:de:0009-7-43402

FanCons.com. (2021). Vic Mignogna information. Retrieved September 13, 2021, from https:/fancons.com/guests/bio/517/vic-mignogna

Flowerdew, J., \& Richardson, J. E. (2017). Introduction. In J. Flowerdew \& J. E. Richardson (Eds.), The Routledge handbook of critical discourse studies (1st ed., pp. 1-10). Routledge. https://doi.org/10.4324/9781315739342

Forchtner, B., \& Kølvraa, C. (2012). Narrating a 'new Europe': From 'bitter past' to selfrighteousness? Discourse \& Society, 23(4), 377-400. https://doi.org/10.1177/0957926512441108

Funimation [@Funimation]. (2019, February 11).Everyone, we wanted to give you an update on the Vic Mignogna situation. Following an investigation, Funimation recast Vic Mignogna [Tweet]. Twitter. https://twitter.com/Funimation/status/1095087396209770501

Gabrielatos, C., McEnery, T., Diggle, P. J., \& Baker, P. (2012). The peaks and troughs of corpus-based contextual analysis. International Journal of Corpus Linguistics, 17(2), 151-175. https://doi.org/10.1075/ijcl.17.2.01gab

Gallagher, R. J., Reagan, A. J., Danforth, C. M., \& Dodds, P. S. (2018). Divergent discourse between protests and counter-protests: \#BlackLivesMatter and \#AllLivesMatter. PLOS ONE, 13(4), e0195644. https://doi.org/10.1371/journal.pone.0195644 
Gee, J. P. (2005). Semiotic social spaces and affinity spaces: From The Age of Mythology to today's schools. In D. Barton \& K. Tusting (Eds.), Beyond communities of practice: Language power and social context (Learning in doing: Social, cognitive and computational perspectives, pp. 214-232). Cambridge University Press. https://doi.org/10.1017/CBO9780511610554.012

Giaxoglou, K. (2018). \#JeSuisCharlie? Hashtags as narrative resources in contexts of ecstatic sharing. Discourse, Context \& Media, 22, 13-20. https://doi.org/10.1016/j.dcm.2017.07.006

Grigsby, S. (2019, August 3). Anime gets its \#MeToo moment in clash between Dallasarea voice actors. The Dallas Morning News. https://www.dallasnews.com/news/courts/2019/08/03/anime-gets-its-metoomoment-in-clash-between-dallas-area-voice-actors/

Gries, S. Th. (2009). What is corpus linguistics? Language and Linguistics Compass, 3(5), 1225-1241. https://doi.org/10.1111/j.1749-818X.2009.00149.x

Hawksey, M. (2014, October 2). Need a better Twitter archiving Google sheet? TAGS v6.0 is here! MASHe. https://hawksey.info/blog/2014/10/need-a-better-twitterarchiving-google-sheet-tags-v6-0-is-here/

Hunston, S. (2008). Collection strategies and design decisions. In A. Lüdeling and M. Kytö (Eds.), Corpus linguistics: An international handbook (Vol. 1, pp. 154-168). Walter de Gruyter. 
Heyd, T., \& Puschmann, C. (2017). Hashtagging and functional shift: Adaptation and appropriation of the \#. Journal of Pragmatics, 116, 51-63.

https://doi.org/10.1016/j.pragma.2016.12.004

Iqbal, M. (2021, May 4). Twitter revenue and usage statistics (2021). Business of Apps. https://www.businessofapps.com/data/twitter-statistics/

Kan, M. (2020, April 28). Twitter retires SMS-based tweets and notifications, citing security. PCMag. https://www.pcmag.com/news/twitter-retires-sms-based-tweetsand-notifications-citing-security

Kastrenakes, J. (2020, April 27). Twitter turns off its original SMS service in most countries. The Verge. https:/www.theverge.com/2020/4/27/21238131/twittersms-notifications-disabled-jack-dorsey-hack

KhosraviNik, M. (2017). Social media critical discourse studies (SM-CDS). In J. Flowerdew \& J. E. Richardson (Eds.), The Routledge handbook of critical discourse studies (1st ed., pp. 582-596). Routledge. https://doi.org/10.4324/9781315739342

Lazar, M. M. (2007). Feminist critical discourse analysis: Articulating a feminist discourse praxis. Critical Discourse Studies, 4(2), 141-164. https://doi.org/10.1080/17405900701464816

Lazar, M. M. (2017). Feminist critical discourse analysis. In J. Flowerdew \& J. E. Richardson (Eds.), The Routledge handbook of critical discourse studies (1st ed., pp. 372-387). Routledge. https://doi.org/10.4324/9781315739342 
Louw, B. (1993). Irony in the text or insincerity in the writer? The diagnostic potential of semantic prosodies. In M. Baker, G. Francis, \& E. Tognini-Bonelli (Eds.), Text and technology: In honour of John Sinclair (pp. 157-176). John Benjamins.

Loveridge, L. (2019a, February 4). Vic Mignogna no longer a member of RWBY cast. Anime News Network. https://www.animenewsnetwork.com/interest/2019-0204/vic-mignogna-no-longer-a-member-of-rwby-cast/.142946

Loveridge, L. (2019b, February 14). Dub voice actor Vic Mignogna issues statement: 'Taking time to recommit to God, seeking help'. Anime News Network. https://www.animenewsnetwork.com/interest/2019-02-14/dub-voice-actor-vicmignogna-issues-statement-taking-time-to-recommit-to-god-seekinghelp/.143423

Loveridge, L. (2019c, July 29). Mignogna deposition: The Funimation investigation. Anime News Network. https://www.animenewsnetwork.com/interest/2019-0729/mignogna-deposition-the-funimation-investigation/.149250

Machin, D., \& Mayr, A. (2012). How to do critical discourse analysis. Sage.

Martin, J. R., \& White, P. R. R. (2005). The language of evaluation: Appraisal in English. Palgrave Macmillan. https://doi.org/10.1057/9780230511910

Martinez, P. (2019, February 5). Vic Mignogna no longer part of 'RWBY' cast amid allegations. Newsweek. https://www.newsweek.com/vic-mignogna-rwbyallegations-1318722

McEnery, T., Xiao, R. \& Tono, Y. (2006). Corpus-based language studies: An advanced resource book. Routledge. 
Mendes, K., Ringrose, J., \& Keller, J. (2018). \#MeToo and the promise and pitfalls of challenging rape culture through digital feminist activism. European Journal of Women's Studies, 25(2), 236-246. https://doi.org/10.1177/1350506818765318

Palomino-Manjón, P. (2020). Feminist activism on Twitter: The discursive construction of sexual violence and victim-survivors in \#WhyIDidntReport. Journal of Language Aggression and Conflict, 1-29. https://doi.org/10.1075/jlac.00049.pal

Partington, A., \& Marchi, A. (2015). Using corpora in discourse analysis. In D. Biber \& R. Reppen (Eds.), The Cambridge handbook of English corpus linguistics (Cambridge handbooks in language and linguistics, pp. 216-234). Cambridge University Press. https://doi.org/10.1017/CBO9781139764377.013

Pineda, R. A. (2019, February 20). Bulma voice actress Monica Rial shares alleged inappropriate encounters with Vic Mignogna. Anime News Network. https://www.animenewsnetwork.com/interest/2019-02-20/bulma-voice-actressmonica-rial-shares-alleged-inappropriate-encounters-with-vic-mignogna/.143664

Potts, A., \& Baker, P. (2012). Does semantic tagging identify cultural change in British and American English? International Journal of Corpus Linguistics, 17(3), 295324. https://doi.org/10.1075/ijcl.17.3.01 pot

Radulovic, P. (2019, January 25). Dragon Ball Super: Broly voice actor responds to sexual harassment, homophobia claims. Polygon. https://www.polygon.com/2019/1/25/18197827/dragon-ball-super-broly-vicmignogna-harassment-response 
Reisigl, M. (2017). The discourse-historical approach. In J. Flowerdew \& J. E.

Richardson (Eds.), The Routledge handbook of critical discourse studies (pp. 44-59). Routledge.

Rightler-McDaniels, J. L., \& Hendrickson, E. M. (2014). Hoes and hashtags: constructions of gender and race in trending topics. Social Semiotics, 24(2), 175190. https://doi.org/10.1080/10350330.2013.859355

Rodgers, B. (2019, January 14). The man who brought anime to America: Sony Pictures Television's Funimation CEO Gen Fukunaga. Forbes. https://www.forbes.com/sites/brucerogers/2019/01/14/the-man-who-brought anime-to-america-sony-pictures-televisions-funimation-ceo-gen-fukunaga/

Ruest, N., \& Milligan, I. (2016). An open-source strategy for documenting events: The case study of the 42nd Canadian federal election on Twitter. Code $\{4\}$ lib, 32 . Retrieved March 25, 2021, from http://journal.code4lib.org/articles/11358

Saxton, G. D., Niyirora, J., Guo, C., \& Waters, R. (2015). \#AdvocatingForChange: The strategic use of hashtags in social media advocacy. Advances in Social Work, 16(1), 154-169. https://doi.org/10.18060/17952

Scott, K. (2015). The pragmatics of hashtags: Inference and conversational style on Twitter. Journal of Pragmatics, 81, 8-20. https://doi.org/10.1016/j.pragma.2015.03.015

Scott, M. (2009). In search of a bad reference corpus. In D. Archer (Ed.), What's in a word-list?: Investigating word frequency and keyword extraction (1st ed., pp. 7991). Routledge. https://doi.org/10.4324/9781315547411 
Silva, T. (2019, April 23). Text analysis with AntConc for social media data: Intro, files and settings. Tarcízio Silva. https://tarciziosilva.com.br/blog/text-analysis-withantconc-for-social-media-data-exploration-intro-files-and-settings/

Sherman, J. (2019, November 27). Judge rules Vic Mignogna must pay US\$238,042 to defendants in lawsuit. Anime News Network. https://www.animenewsnetwork.com/news/2019-11-27/judge-rules-vicmignogna-must-pay-usd238042-to-defendants-in-lawsuit/.153749

Sports Lesbian@ [@CryptidTrash]. (2019, January 23).Look how long ago this answer to a Yahoo question was posted. Six years ago. Vic Mignogna has been a [Tweet; image attached]. Twitter. https://witter.com/CryptidTrash/status/1088280009138233344

Starr, J. (2017, February 8). Clean up weird characters in database. Digging Into WordPress. https://digwp.com/2011/07/clean-up-weird-characters-in-database/

Stubbs, M. (2001). Words and phrases: Corpus studies of lexical semantics. Blackwell.

Taspinar, A. (2020). TwitterScraper. GitHub. https://github.com/taspinar/twitterscraper

Taylor, C. (2013). Searching for similarity using corpus-assisted discourse studies. Corpora, 8(1), 81-113. http://dx.doi.org/10.3366/cor.2013.0035

Twitter. (n.d.). How to use hashtags. Help Center. Retrieved August 2, 2021, from https://help.twitter.com/en/using-twitter/how-to-use-hashtags

Twitter Support [@TwitterSupport]. (2020, April 27).We want to continue to help keep your account safe. We've seen vulnerabilities with SMS, so we've turned off our 
[Tweet]. Twitter.

https:/twitter.com/TwitterSupport/status/1254822372353257473

van Dijk, T. A. (1998). Ideology: A multidisciplinary approach. Sage.

van Leeuwen, T. (2008). Discourse and practice: New tools for critical discourse analysis. Oxford University Press.

Vander Wal, T. (2007, February 2). Folksonomy coinage and definition. Folksonomy. http://www.vanderwal.net/folksonomy.html

Vessey, R. (2017). Corpus approaches to language ideology. Applied Linguistics, 38(3), 277-296. https://doi.org/10.1093/applin/amv023

Vessey, R. (2019). Domestic work = language work? Language and gender ideologies in the marketing of multilingual domestic workers in London. Gender and Language, 13(3), 314-338. https://doi.org/10.1558/genl.35581

Wade, J. (2019, February 5). Update: Dragon Ball voice actor dismissed from Funimation, RWBY following misconduct allegations. IGN. https://www.ign.com/articles/2019/02/05/dragon-ball-voice-actor-dismissedfrom-rwby-following-misconduct-allegations

Wodak, R., \& Meyer, M. (2015). Critical discourse analysis: History, agenda, theory, and methodology. In R. Wodak \& M. Meyer (Eds.), Methods of critical discourse studies (3rd ed., pp. 1-33).

Zappavigna, M. (2011). Ambient affiliation: A linguistic perspective on Twitter. New Media \& Society, 13(5), 788-806. https://doi.org/10.1177/1461444810385097 
Zappavigna, M. (2015). Searchable talk: The linguistic functions of hashtags. Social Semiotics, 25(3), 274-291. https://doi.org/10.1080/10350330.2014.996948

Zappavigna, M. (2018). Searchable talk: Hashtags and social media metadiscourse. Bloomsbury.

Zhu, H. (2016). Searchable talk as discourse practice on the internet: The case of “\#bindersfullofwomen.” Discourse, Context \& Media, 12, 87-98. https://doi.org/10.1016/j.dcm.2015.10.001

Zuleika. (2019, April 19). Subs vs dubs: The evolution of English anime adaptations. Fandom. Retrieved May 27, 2021, from https:/www.fandom.com/articles/subsvs-dubs-english-anime-adaptations 